Document downloaded from:

http://hdl.handle.net/10251/37358

This paper must be cited as:

Salinas Soler, Y.; Martínez Mañez, R.; Marcos Martínez, MD.; Sancenón Galarza, F.; Costero Nieto, AM.; Parra Alvarez, M.; Gil Grau, S. (2012). Optical chemosensors and reagents to detect explosives. Chemical Society Reviews. 41(3):1261-1296. doi:10.1039/c1cs15173h.

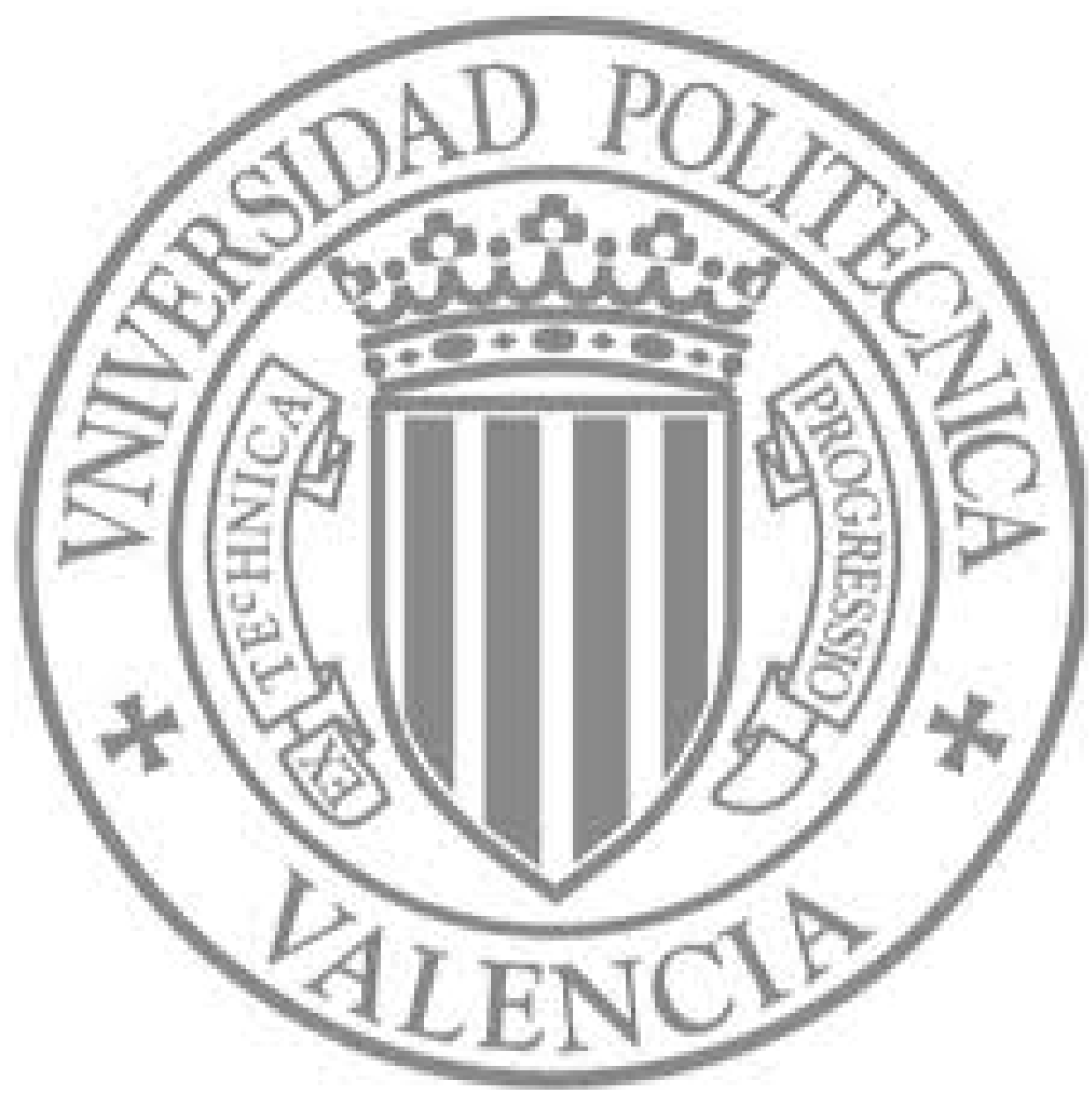

The final publication is available at

http://dx.doi.org/10.1039/C1CS15173H

Copyright Royal Society of Chemistry 


\section{Optical chemosensors and reagents to detect explosives}

Yolanda Salinas, ${ }^{\text {a,b,c }}$ Ramón Martínez-Máñez, ${ }^{\mathrm{a}, \mathrm{b}, \mathrm{c} *}$ María D. Marcos, ${ }^{\mathrm{a}, \mathrm{b}, \mathrm{c}}$

Félix Sancenón, ${ }^{\mathrm{a}, \mathrm{b}, \mathrm{c}}$ Ana M. Costero, ${ }^{\mathrm{a}, \mathrm{d} *}$ Margarita Parra, ${ }^{\mathrm{a}, \mathrm{d}}$ Salvador Gil ${ }^{\mathrm{a}, \mathrm{d}}$

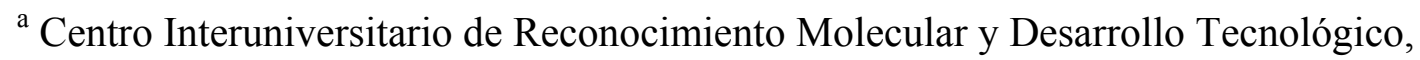
(IDM), Unidad Mixta Universidad Politécnica de Valencia - Universitat de València, Spain.

${ }^{\mathrm{b}}$ Departamento de Química. Universidad Politécnica de Valencia. Camino de Vera s/n. E-46022, Valencia, Spain.

${ }^{c}$ CIBER de Bioingeniería, Biomateriales y Nanomedicina (CIBER-BBN).

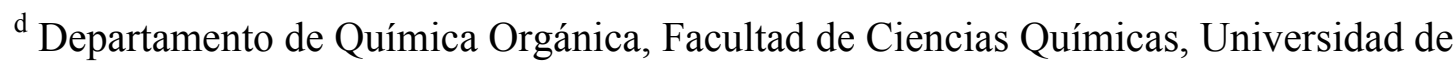
Valencia. Doctor Moliner 50, 46100 Burjassot, Valencia, Spain.

This review is focused on examples reported from 1947 to 2010 related to the design of chromo-fluorogenic chemosensors and reagents for explosives.

\section{1.- Introduction}

In the last few years, the reliable and accurate detection of explosives is an issue of international concern. The detection of chemical explosives is crucial for homeland security, environmental cleaning and military issues. Additionally, given the widespread use of explosive formulations, the analysis of explosives is also of interest in forensic research, land mine detection, and in the study of environmental problems associated with explosive residues. The recent rise in global terrorism has required that the methods followed to detect explosives should be both sensitive and low-cost. With all this in mind, trained canine teams, ${ }^{1}$ gas chromatography coupled with mass spectrometry, ${ }^{2}$ gas chromatography-electron capture detection, ${ }^{3}$ surface-enhanced Raman spectroscopy, ${ }^{4}$ mass spectrometry, ${ }^{5}$ X-ray imaging, ${ }^{6}$ thermal neutron analysis, ${ }^{7}$ electrochemical procedures ${ }^{8}$ and ion mobility spectroscopy (IMS) ${ }^{9}$ have been used or proposed as suitable methods for the detection and quantification of these chemicals. While all the above-mentioned methods offer advantages, none is ideal due to certain features such as lack of portability, susceptibility to false positives owing to 
environmental contaminants, and false-negative readings due to certain interfering compounds. $^{10}$

Very recently, optical detection techniques based on the design of colorimetric and fluorimetric assays have attracted a great deal of attention. ${ }^{11}$ In particular, the wide variety of fluorescent and dye groups, the simple instrumentation required when using colorimetric probes, and the usually low detection limit reached when employing fluorescence probes, all make the optical approach largely appealing. In fact, the development of chromogenic and fluorogenic chemosensors and reagents is an area that is emerging in the supramolecular field, and different protocols have been widely applied to the design of probes for anions, ${ }^{12}$ cations ${ }^{13}$ and, to a lesser extent, to neutral molecules. ${ }^{14}$ Chemosensors are systems that rely on the use of coordinative forces for guest binding. However, we will use the term reactand (or chemodosimeter) when the signalling event uses specific irreversible reactions. ${ }^{15}$ In this context, a relatively large number of signalling chemosensors and reactands has been reported for the optical detection of explosives. 
<smiles>O=[N+]([O-])c1cc([N+](=O)[O-])c(O)c([N+](=O)[O-])c1</smiles>

PA<smiles>CN(c1c([N+](=O)[O-])cc([N+](=O)[O-])cc1[N+](=O)[O-])[N+](=O)[O-]</smiles>

Tetryl<smiles>Cc1c([N+](=O)[O-])cc([N+](=O)[O-])cc1[N+](=O)[O-]</smiles>

TNT<smiles>Cc1ccc([N+](=O)[O-])cc1[N+](=O)[O-]</smiles>

2,4-DNT<smiles>Cc1c([N+](=O)[O-])cccc1[N+](=O)[O-]</smiles>

2,6-DNT<smiles>Cc1ccc([N+](=O)[O-])cc1[N+](=O)[O-]</smiles><smiles>O=[N+]([O-])c1ccc(O)c([N+](=O)[O-])c1</smiles><smiles>O=[N+]([O-])c1cccc2ccccc12</smiles><smiles>Cc1cc(C)cc([N+](=O)[O-])c1</smiles><smiles>Cc1cccc(C)c1[N+](=O)[O-]</smiles><smiles>O=[N+]([O-])c1cccc2ncccc12</smiles><smiles>O=[N+]([O-])c1ccccc1Cl</smiles>

NP

NN

5-NX

NX

NQ

CINB<smiles>NC(C(=O)C(Cl)Cl)C(CO)C(O)c1ccc([N+](=O)[O-])cc1</smiles><smiles>CC(C(C)[N+](=O)[O-])[N+](=O)[O-]</smiles><smiles>C[N+](=O)[O-]</smiles><smiles>CC[N+](=O)[O-]</smiles>

ClAmp

DMNB

NM

NE<smiles>O=[N+]([O-])N1CN([N+](=O)[O-])CN([N+](=O)[O-])C1</smiles>

RDX<smiles>O=[N+]([O-])N1CN([N+](=O)[O-])CN([N+](=O)[O-])CN([N+](=O)[O-])C1</smiles>

HMX<smiles></smiles>

PETN<smiles>O=[N+]([O-])OCC(CO[N+](=O)[O-])O[N+](=O)[O-]</smiles>

TNG<smiles>O=C(OOC(=O)c1ccccc1)c1ccccc1</smiles>

BP<smiles>CC1(C)OOC(C)(C)OOC(C)(C)OO1</smiles>

TATP<smiles>CC1(O)COCN(CO)COOCN1CO</smiles>

HMTD

Fig. 1 Chemical structures of explosives 
Explosive compounds are classified mainly into four classes: (i) nitroaromatics and nitroalkanes (ii) nitramines; (iii) nitrate esters; and, finally, (iv) peroxides (see Fig. 1 for the chemical structure of the explosives that appear in this review). ${ }^{16}$ Nitroaromatic explosives (such as TNT, 2,4-DNT and PA) are composed of a benzene ring functionalised with several nitro groups. The detection of this class of explosives is not easy because they have moderate vapour pressures and their reactivity is quite limited. These electron-deficient molecules are able to form $\pi$-stacking complexes with electron-rich fluorophores, and this particular property has been widely used for their detection with chromo-fluorogenic probes. Nitroalkanes, such as DMNB, are explosive taggants that present weak binding interactions with electron-rich derivatives, thus making their detection with supramolecular-based interactions extremely difficult. Nitramines (such as RDX) and nitrate esters (PETN) are aliphatic explosives with N$\mathrm{NO}_{2}$ and $\mathrm{O}-\mathrm{NO}_{2}$ moieties, respectively. Although the lack of aromatic rings in both types makes their optical detection challenging, their unique functional groups allow detection through chemical reactions. Finally, peroxide-based explosives are easily synthesised from commercially available and inexpensive materials. In most cases, the strong oxidant character of these explosives has been used to design fluorogenic and chromogenic probes based on redox reactions coupled by colour/emission changes.

This review aims to be a comprehensive recompilation of the examples reported in the literature relating to the design of chromogenic and fluorogenic chemosensors and of reagents to recognise explosive molecules. The examples provided in this review are related to the application of supramolecular-coordination concepts for the development of optical receptors, and they do not cover optical sensors based on the use of biomolecules. Examples based on irreversible chemical reactions (the chemodosimeter approach) have also been included. This review has been divided into two main sections in relation with the design of (a) fluorescent/luminescent and (b) colorimetric probes. Each section is additionally divided to cover the type of chromo-fluorogenic group used to prepare the final sensing system.

\section{2.- Fluorescence and luminescent sensors for explosives}

\section{1.- Fluorescent Conjugated Polymers}


Fluorescent-conjugated polymers have been employed extensively as sensing materials in recent years. ${ }^{17,18}$ One of the features that have boosted the use of fluorescent-conjugated polymers as sensors relates to their ability to produce signal gain in response to an interaction with target analytes. Signal amplification is a direct consequence of the conjugated polymer's ability to serve as a highly efficient transport medium via the migration of excited states (excitons) through the polymer chain by mechanism that involves through space dipolar couplings and/or a strong mixing of electronic states. ${ }^{19,20}$ The first examples of amplifying polymer were developed by the research group of Swager using poly(phenylene ethynylene) with cyclophane receptors integrated in the polymeric backbone for coordination with paraquat (a powerful quencher). ${ }^{21}$ Since this work emerged, fluorescence-amplifying polymers have been extensively employed as sensing materials to detect metal cations, anions and neutral molecules. Explosives are included in the large family of neutral chemical species. The fact that many explosives are nitrated organic compounds with a marked electrondeficient character makes them efficient quenchers of fluorescence through photoinduced electron transfer processes. For these reasons, ever increasing interest has been paid to the development of fluorescent-conjugated amplifying polymers for the detection of explosives.

A number of examples have been reported, most of which are based on the use of poly(phenylene ethynylene)s, poly(phenylene vinylene)s and Si-containing polymers. This section also includes some examples of molecular imprinting polymers (MIPs) for the detection of explosives.

\subsection{1.- Poly(phenylene ethynylene)s}

Poly(phenylene ethynylene)s are conjugated polymers that have been widely used as active materials in fluorescence-based chemical sensors because of their high sensitivity to a variety of solution- and vapour-phase analytes. In the specific field of sensing explosives, poly(phenylene ethynylene)s polymers have been widely employed since the pioneering work of T. M. Swager et al. In most cases, polymers are used as films. In this case, the materials' response characteristics depend on a number of parameters, including the strength of the chemical (or physical) interaction between the analyte and the photoactive polymer, and the permeability of the analyte in the polymer. In this sense, Swager et al have additionally shown that the use of the sterically demanding pentiptycene moiety increases the permeability of the fluorescent 
poly(phenylene ethynylene)s polymers films, thus increasing the material's response to vapour-phase explosives. Additionally, the incorporation of the rigid three-dimensional pentiptycene molecule into the polymeric backbone prevents low quantum yields due to $\pi$-stacking, excimer formation and self quenching, which usually show some other fluorescent polymers. A bulky pentiptycene moiety is believed to create molecular-scale channels, which provide pathways for the analyte molecules to diffuse and readily interact with the electron-rich $\pi$-conjugated system in the polymer. This resulting increase in permittivity has been reported to allow nitroaromatics to quench the polymer's fluorescence more rapidly and more efficiently compared to similar polymeric materials lacking the sterically-demanding groups. In all the following examples, the quenching mechanism is similar, and involves an electron transfer process between the corresponding excited polymer and electron acceptor nitroaromatic explosive molecules.

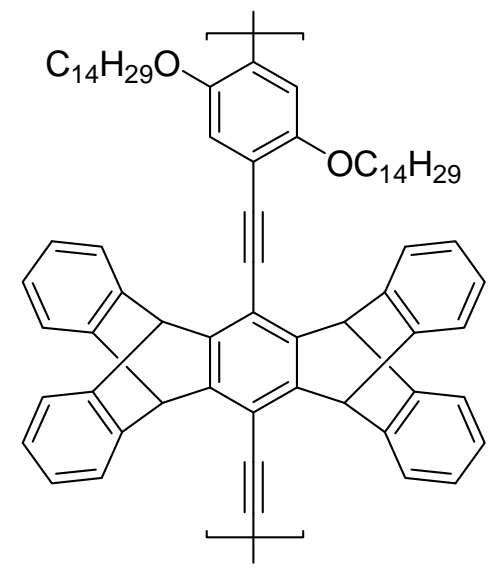

1

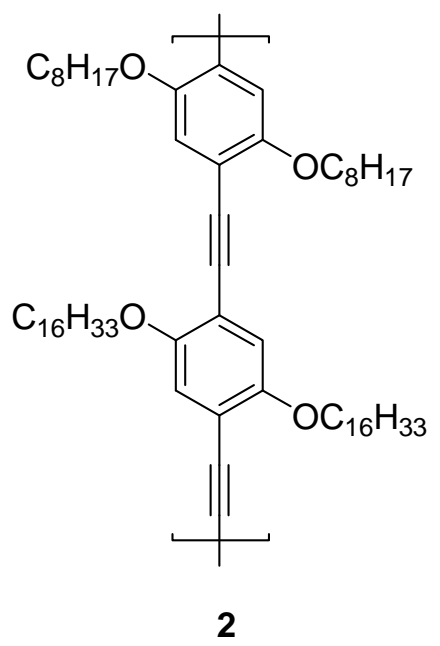

2

Fig. 2 Compounds 1 and 2

Along these lines, T. M. Swager et al prepared polymers 1 and 2 (Fig. 2), and showed how they underwent severe fluorescence quenching in the presence of TNT and 2,4-DNT vapours. ${ }^{22}$ The intensity of emission quenching was influenced by the exposure time and by thin film thickness. The best results were obtained with films of $25 \AA$ and with exposure times of 60 seconds (100\% and 75\% quenching with 2,4-DNT and TNT, respectively, for 1-containing films). 


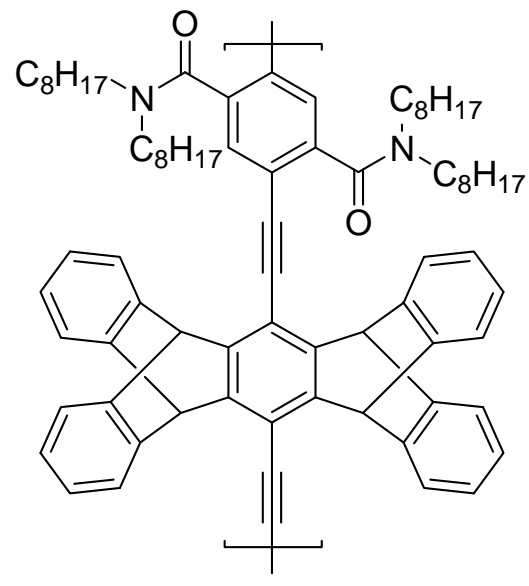

3

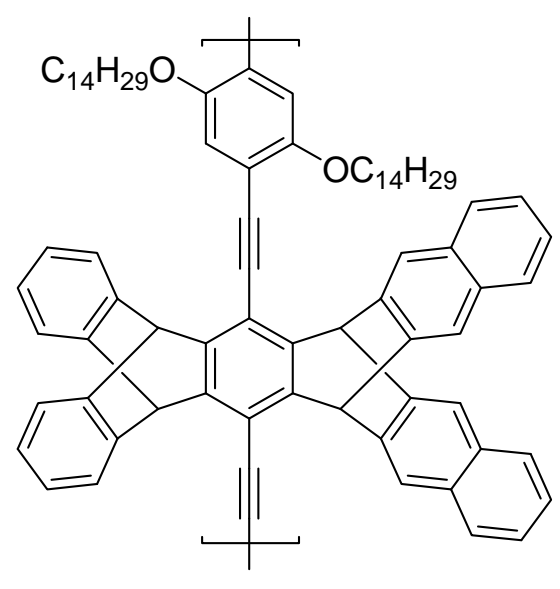

4

Fig. 3 Compounds 3 and 4

In an extended version of their previous work, Yang and Swager synthesised pentiptycene-containing polymers 3 and 4 (Fig. 3) for the vapour-phase sensing of nitroaromatic compounds. ${ }^{23}$ A $200 \AA$ film of polymer 3 showed an emission band at $423 \mathrm{~nm}$, whereas the films of polymer 4 exhibited two emission bands at 463 and 497 $\mathrm{nm}$; the latter was assigned to the formation of an excimer. The fluorescence of the thin films of both polymers was quenched, be it to different extents, in the presence of nitroaromatic derivatives such as TNT, 2,4-DNT, 2,4-DNB and NB. Quenching efficiencies were higher for polymer $\mathbf{4}$ than for $\mathbf{3}$ due to the electron-withdrawing character of the amide groups in the structure of $\mathbf{3}$. The amide groups make polymer 3 less electron donor, when compared with 4. Moreover, the interaction with the nitroaromatic compound was weaker, leading to moderate quenching efficiencies. 

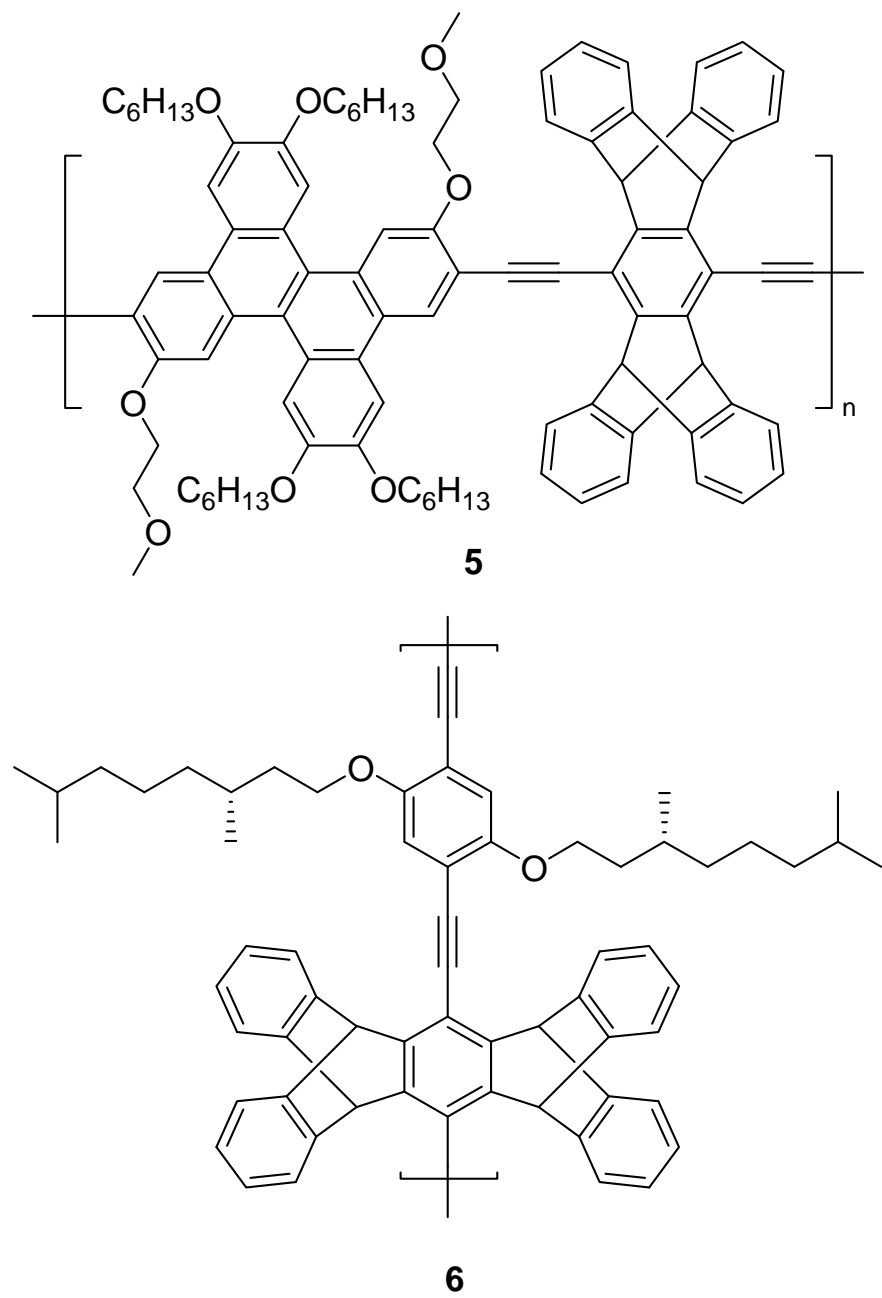

Fig. 4 Compounds 5 and 6

The related pentiptycene-containing polymers 5 and 6 (Fig. 4) also displayed sensing ability for TNT. ${ }^{24,25}$ The films of 5 presented an emission band at $474 \mathrm{~nm}$, which was quenched in the presence of TNT vapours. This polymer showed higher sensitivity to TNT than that observed for polymers 1-4. The films of 6 presented an intense emission band at $475 \mathrm{~nm}$, which was severely quenched (by about 75\%) upon exposure to $10 \mathrm{ppb}$ of the TNT vapours. 


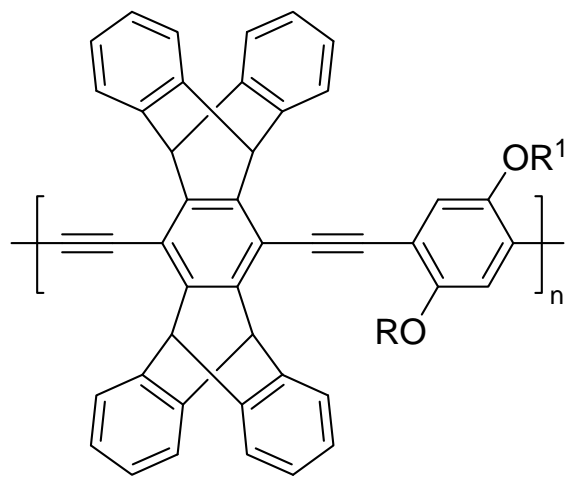

$7 \mathrm{R}, \mathrm{R}^{1}=\mathrm{C}_{16} \mathrm{H}_{33}$

$8 \mathrm{R}=\mathrm{C}_{10} \mathrm{H}_{21} \quad \mathrm{R}^{1}={ }_{\mathrm{CF}_{3}}^{\mathrm{CH}}$

$9 \mathrm{R}, \mathrm{R}^{1}=\overbrace{\mathrm{CF}_{3}}^{\mathrm{OH}}$

Fig. 5 Compounds 7, 8 and 9

Swager et al. also reported the design of the polyacetylene pentiptycenecontaining polymers 7-9 (Fig. 5) and tested their emission behaviour in the presence of electron deficient molecules. ${ }^{26}$ Polymers 8 and $\mathbf{9}$ contain pendant hexafluoro-2-propanol groups, therefore displaying a strong hydrogen-bonding donating character. The exposure of polymers 7-9 to the vapours of 2,4-DNT brought about a moderate quenching of the emission intensities (between 16\% and 28\%). Almost the same response was observed in the presence of the vapours of 2,4-dichloropyrimidine for all three polymers. In addition to the usual $\pi$-stacking interactions between these polymers and analytes, a coordinate interaction via hydrogen-bonding forces between electrondeficient molecules and the hexafluoro-2-propanol moiety also occurred for polymers $\mathbf{8}$ and 9. 


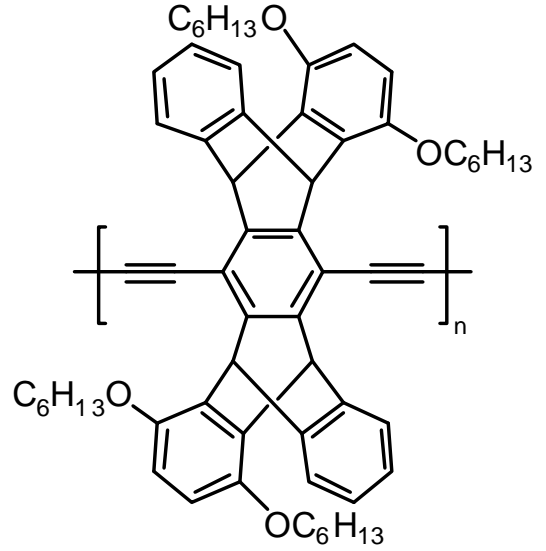

10

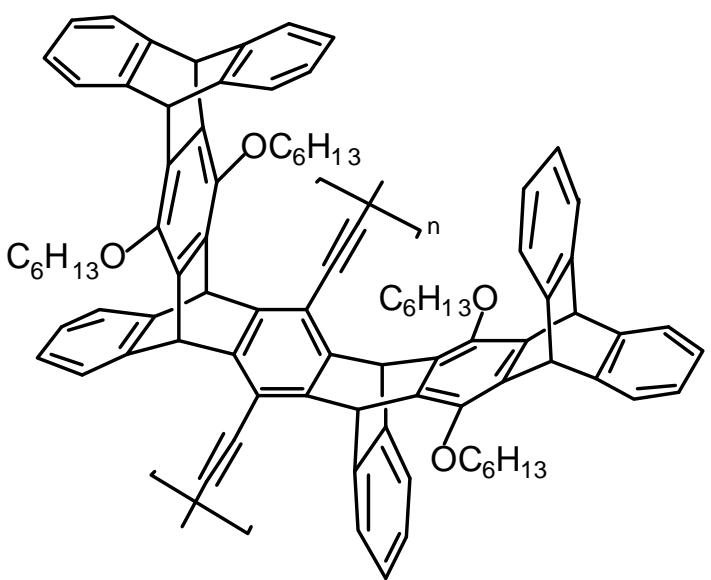

12

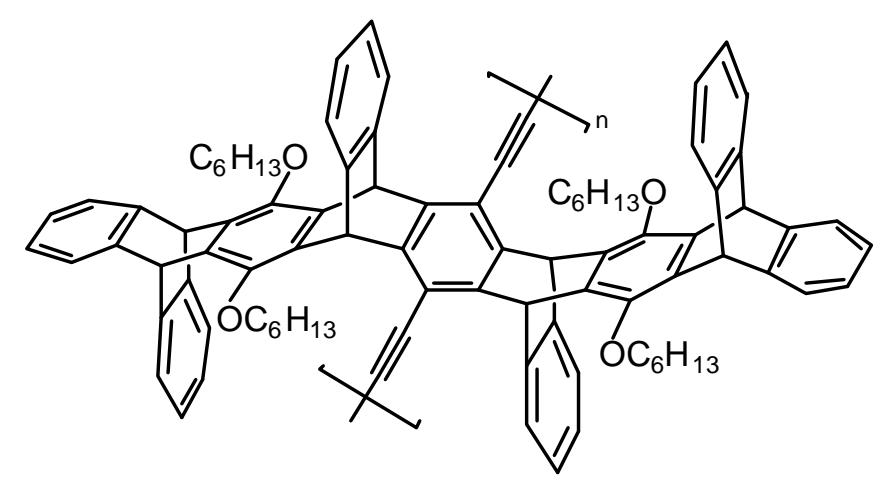

11

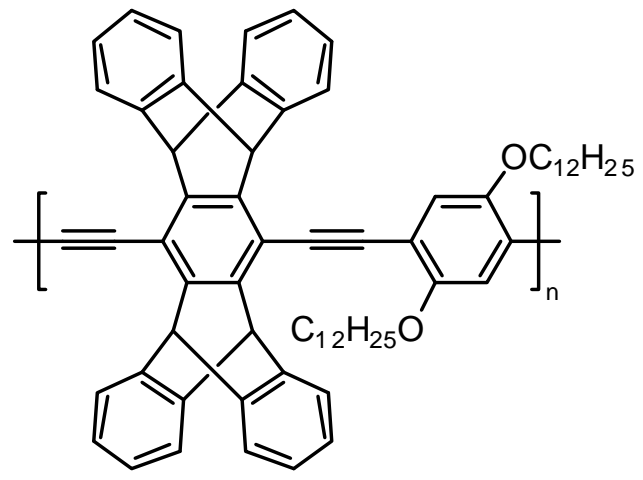

13

Fig. 6 Compounds 10, 11, 12 and 13

Following the use of the pentiptycene-containing polymers to recognise nitroaromatic compounds, Swager's research group recently prepared derivatives 10-13 (Fig. 6). ${ }^{27}$ In a chloroform solution, polymers 10-12 showed intense emission bands in the 420-430 nm interval, which were effectively quenched upon addition of TNT, 2,4DNT and 4-NT. The most efficient quencher was TNT, while 4-NT was the least effective. Virtually the same response was observed for polymer $\mathbf{1 3}$ in a chloroform solution. Thin films of all four polymers were prepared by spin-casting. The emission of the films of 10-13 was quenched upon the addition of TNT and 2,4-DNT (quenching was lower for 10-12 than for 13). 

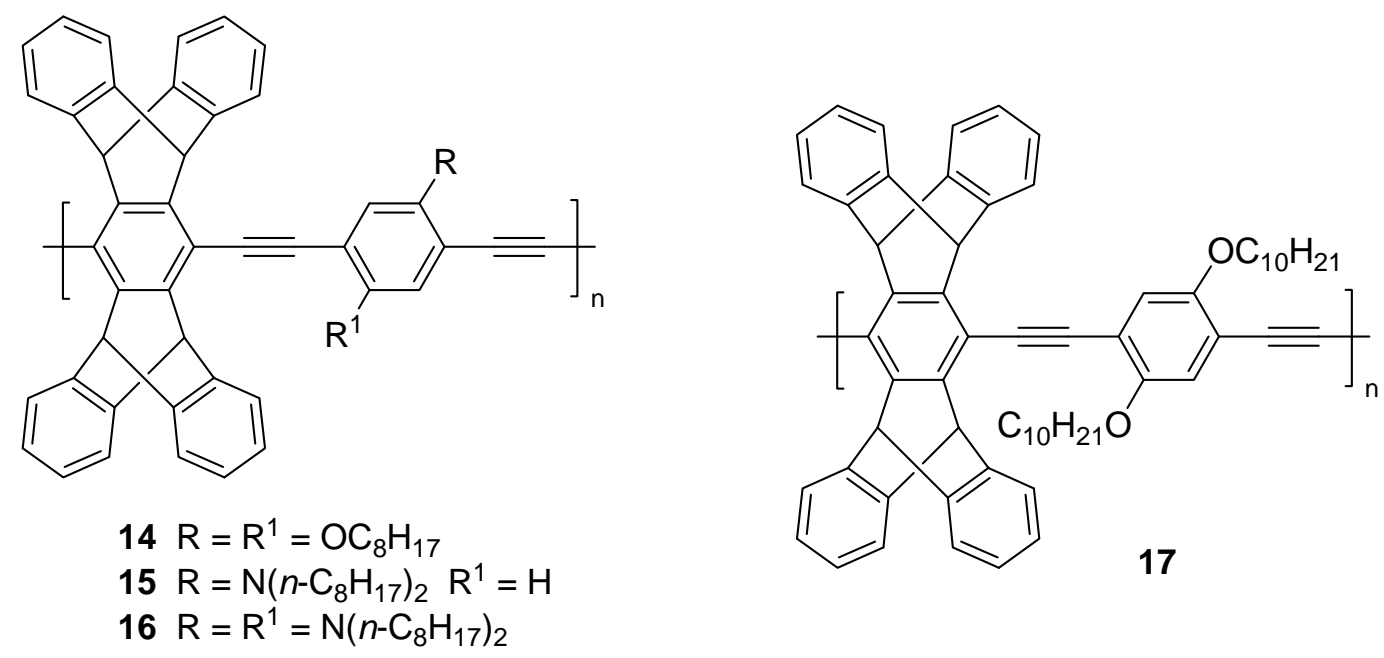

Fig. 7 Compounds 14, 15, 16 and 17

Polyacetylene pentiptycene-containing polymers 14-16 (Fig. 7) have been used for the fluorescent sensing of DMNB, which is an additive required in all legally manufactured plastic explosives. ${ }^{28}$ The thin films of poly(phenylene ethynylene) polymers 14-16 showed very weak fluorescent quenching (about 5\% for 15) in the presence of DMNB vapours. The moderate quenching observed with DMNB, when compared with those obtained in the presence of nitroaromatic compounds, was ascribed to the three-dimensional shape of this compound, and to the lack of $\pi$-stacking interactions with the polymer chains that induced weak binding.

Polyacetylene-containing polymer 17 exhibited multi-photon excitation and was used for the fluorescent recognition of TNT. ${ }^{29}$ In line with this, the two- or three-photon excitation of the solutions of 17 displayed an emission band at $460 \mathrm{~nm}$, which was quenched in the presence of TNT. 


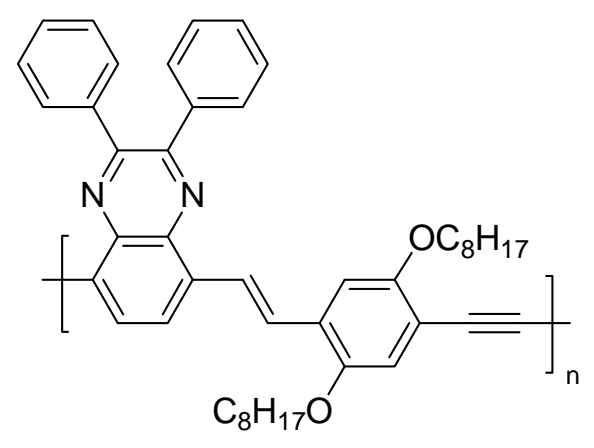

18

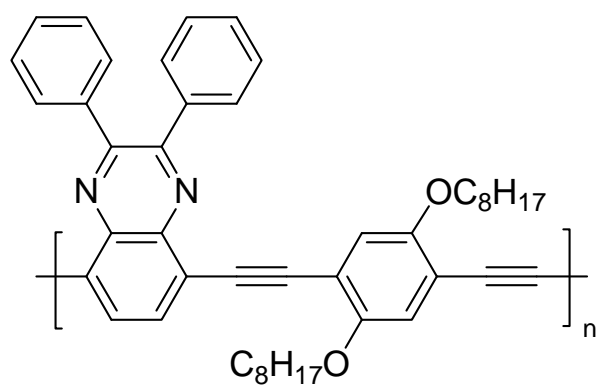

19

Fig. 8 Compounds 18 and 19

Apart from the remarkable work of Swager et al using polyacetylene pentiptycene-containing polymers, polyacetylene derivatives containing quinoxaline and dibenz $[a, h]$ anthracene groups have also been reported for the fluorogenic sensing of nitroaromatic explosives. Zhang et al reported the emission quenching of quinoxalinecontaining polymers 18 and 19 (Fig. 8) in the presence of TNT. ${ }^{30}$ The chloroform solutions of both polymers displayed intense emission bands at 620 and at $500 \mathrm{~nm}$ for 18 and 19, respectively, which were gradually quenched upon the addition of increasing amounts of TNT. The same quenching effect in the presence of TNT vapour was observed when using the films of 18 and 19. The authors observed a more efficient quenching when using polymer 19. They ascribed this feature to the fact that this polymer has a more planar backbone if compared with polymer 18, which enables easier exciton migration.

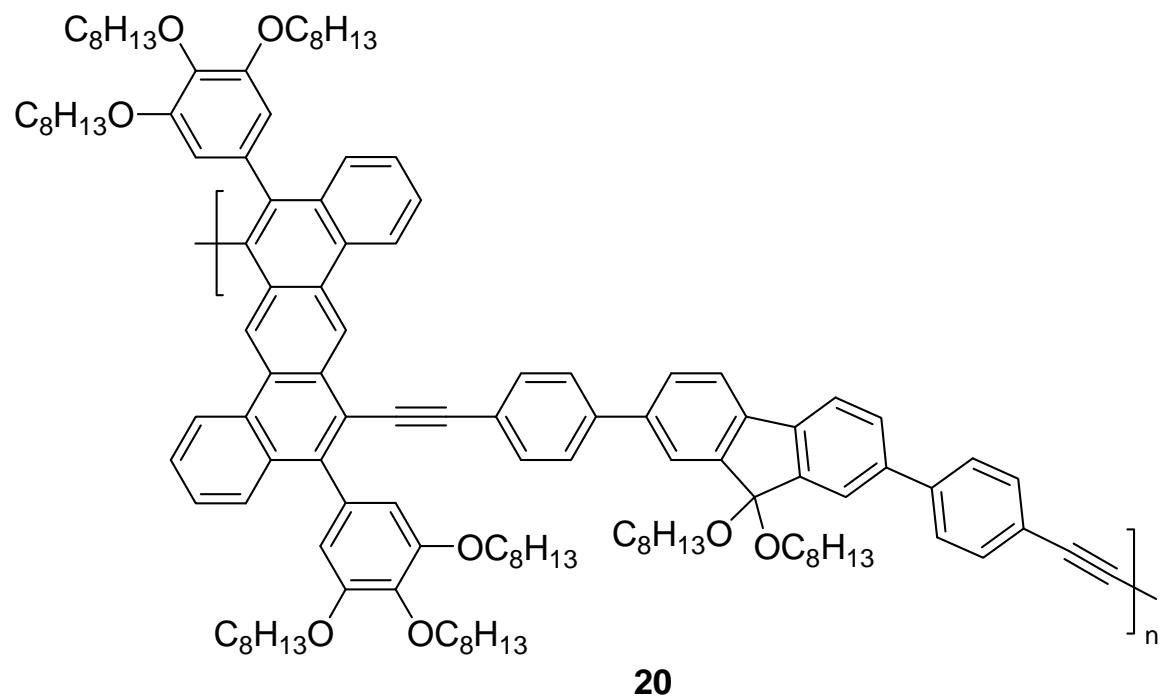

Fig. 9 Compounds 20 
Additionally, Liu et al reported the use of dibenz $[a, h]$ anthracene-containing polymer 20 (Fig. 9) for the detection of 2,4-DNT. 20 showed two intense emission bands at 427 and $453 \mathrm{~nm}$ in chloroform, which were significantly quenched in the presence of 2,4-DNT. ${ }^{31}$ As in the aforementioned cases, the quenching effect was due to a photo-induced electron transfer process favoured by the formation of a complex between the electron-rich polycyclic backbone of $\mathbf{2 0}$ with the electron-deficient 2,4DNT through $\pi-\pi$ stacking interactions. The films of $\mathbf{2 0}$ were prepared by spin-casting or electrospinning using polystyrene as a supporting matrix. Emission of both types of films was quenched upon exposure to 2,4-DNT vapours, but the degree of quenching was greater for the electrospinning film (50\% after a 1 -h exposure) than for the spincasting film (about 5\% for the same exposure time). This remarkable difference in sensing performance was ascribed to the formation of a nanofibrous-sensing film with a large surface area and unique porous structure by means of the electrospinning technique. The film's sensitivity was further improved by introducing a secondary porous system into the nanofibres through the addition of sodium dodecylsulphate as a porogen agent during the electrospinning process.

\subsection{2.- Polyphenylene vinylenes}

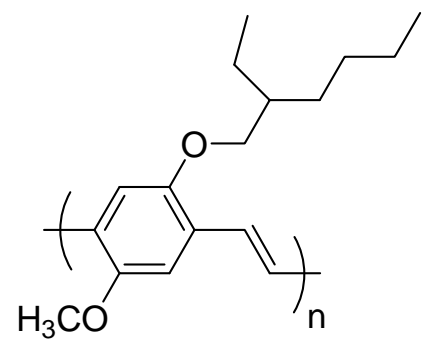

21

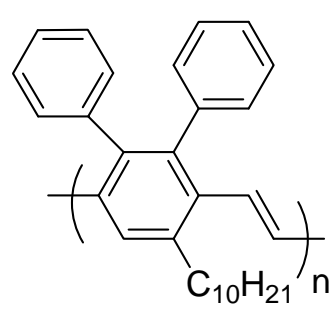

22

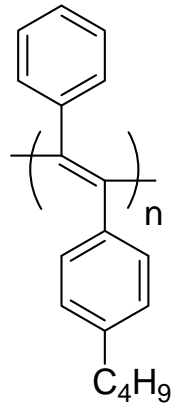

23

Fig. 10 Compounds 21, 22 and 23

Chemosensing applications based on the quenching of light-emitting poly(phenylene vinylene) polymers in the presence of nitroaromatic vapours has also been widely used for the detection of explosive derivatives. For instance, the thin films of polymers 21 and 22 (Fig. 10) on a cover glass show intense emission bands at 574 and $488 \mathrm{~nm}$, respectively, which were quenched in the presence of TNT vapours. ${ }^{32}$ The 
highest quenching efficiency was measured for the thin films of 21 (90\% after 1000 seconds of exposure) and was ascribed to the strong polar-polar interaction of the donor moieties of 21 (not present in 22) with the nitroaromatic molecule. Poly(diphenylacetylene) derivative 23 was also studied. In this case, the emission band at $517 \mathrm{~nm}$ was also quenched in the presence of TNT vapours. Other nitroaromatic compounds, such as 2,4-DNT, 2,6-DNT and 4-NT, also led to a quenching of the emission intensity of the three polymers. As in other similar cases, emission quenching was ascribed to a photo-induced electron transfer between the excited polymer and the nitroaromatic explosive.

The response of $\mathbf{2 1}$ has also been modulated by entrapping the polymer in porous silicon microcavities. ${ }^{33}$ In this situation, the fluorescence spectra of polymers were modulated by a microcavity via a spectral "hole" that matches the resonance peak of microcavity reflectance. With TNT vapours present, a red shift (2-3 nm) of the resonance peak occurred along with fluorescence quenching. The authors indicated that this provided the opportunity for multiple monitoring parameters to help develop an optical sensor array as fluorescence attenuated differently at various wavelengths if compared to the uniform quenching of polymers deposited on a flat substrate.

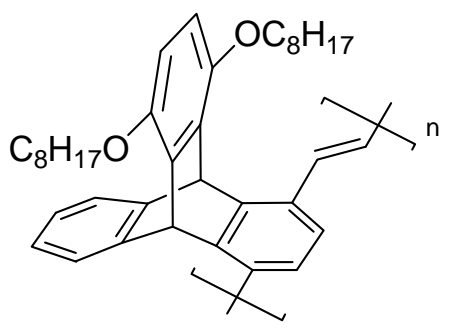

24

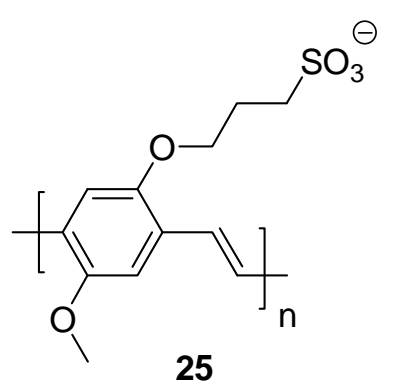

25

Fig. 11 Compounds 24 and 25

Polyphenylene vinylene polymer 24 (Fig. 11) presented bulky iptycene moieties in the backbone, which minimised the interaction between chains and prevented selfquenching. ${ }^{29}$ Besides, the presence of iptycene moieties enhanced the permeability of the polymer by increasing the material's response to nitroaromatic explosives. In line with all this, 24 showed an intense emission band (a quantum yield of 0.76) centred at $477 \mathrm{~nm}$, which was quenched in the presence of TNT. 
Water soluble poly(2,5-methoxy-propyloxysulphonate phenylene vinylene) (25) polymers have also been reported to act as optical chemosensors for nitroaromatics. ${ }^{34}$ The aqueous solutions of $\mathbf{2 5}$ presented an intense emission band, which was severely quenched in the presence of methyl viologen $\left(\mathrm{MV}^{2+}\right)$ due to the strong association between the negatively-charged polymer and the positively-charged quencher. Under these conditions, neutral molecules such as TNT and 2,6-DNT presented quenching sensitivities of three orders of magnitude lower than that of $\mathrm{MV}^{2+}$. Quenching behaviour was modulated by the addition of a cationic surfactant (dodecyltrimethylammonium bromide, DTA). In relation to this, DTA was able to neutralise the negative charge of 25 and to also create a hydrophobic environment around the polymer chains. Under these conditions, quenching in the presence of $\mathrm{MV}^{2+}$ was less effective, whereas quenching by nitroaromatic derivatives was enhanced. Thus, aqueous solutions of 25 and DTA in a 3:1 ratio provided an intense emission, which was quenched efficiently upon the addition of TNT and 2,6-DNT. Similarly, the thin films of 25 and a cationic polyelectrolyte (DTA or PDDA) were successfully used for the detection of 2,6-DNT vapours.

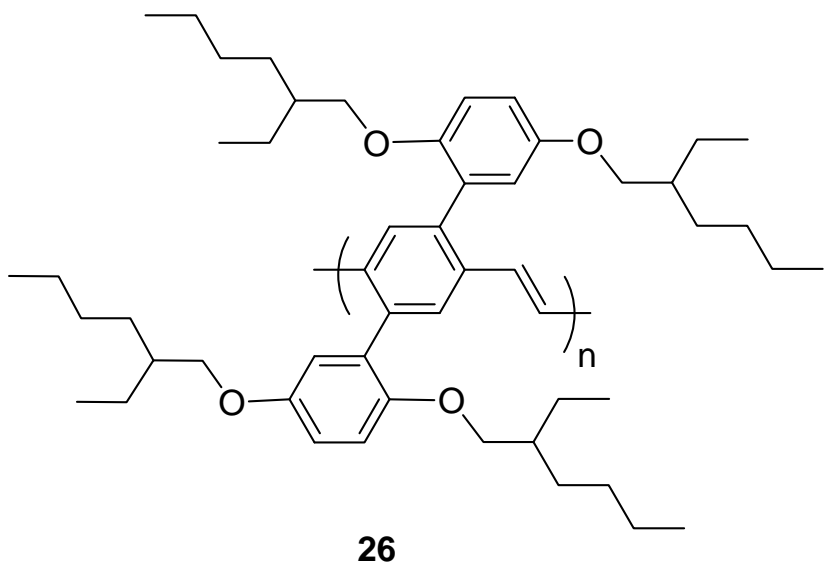

Fig. 12 Compound 26

Semiconducting organic polymer 26 (Fig. 12) was synthesised and used for the fluorescent sensing of TNT and 2,4-DNT vapours. ${ }^{35}$ The thin films of 26 gave an intense emission band at $500 \mathrm{~nm}$, which was quenched in the presence of TNT and 2,4DNT. For the fabrication of solid sensors with amplified signalling, three devices were constructed: (i) the spin-coated films of $\mathbf{2 6}$ on a transparent parylene, forming a twolayer index-matched waveguide on glass; (ii) the spin-coated films of $\mathbf{2 6}$ on distributed feedback gratings fabricated from polydimethylsiloxane; and (iii) the dip coating of 
polymer 26 on a silica optical fibre. Upon the excitation of these devices with a 4-ns nitrogen laser operating at a frequency of $30 \mathrm{~Hz}$, a multimode lasing action was generated and an intense amplified emission was observed. This emission was quenched in the presence of TNT and 2,4-DNT, whose sensitivity was 30 times greater than that observed with the single polymer. The selectivity of the response was based on the strong binding of analytes to electron-rich polymers. No interference was observed with benzene or naphthalene.

\subsection{3.- Si-containing polymers.}

Within the family of Si-containing polymers, silole- and silafluorene-containing polymers have been reported for the fluorogenic detection of organic nitro derivatives. Polysiloles are conjugated polymers with a highly delocalised structure which contain the silacyclopentadiene (silole) group. Siloles proved to be of considerable current interest due to their unusual electronic and optical properties, and because of their possible applications as electron transporting materials in certain devices. ${ }^{36}$ These polymers are highly luminescent and have been used as light-emitting diodes (LEDs) ${ }^{37}$ or as chemical sensors. ${ }^{38}$ The characteristic features of polysiloles include a low reduction potential, and a low-lying LUMO which leads to visible fluorescence due to $\sigma^{*} \rightarrow \pi^{*}$ conjugation arising from the interaction between the $\sigma^{*}$ orbital of the silicon chain and the $\pi^{*}$ orbital of the butadiene moiety of the five-member ring. The usual high sensitivity of these polymers is related with favourable analyte-polymer hydrophobic interactions. Electron delocalisation in these polysiloles provides amplification because the interaction of an analyte molecule at any position along the polymeric chain quenches an excited state delocalised along the chain.

Silafluorene-containing polymers presented similar electronic features to those of polysiloles, but the presence of a biphenyl framework increased the band gap energy allowing UV-blue emission. Normally, silafluorene moiety was incorporated into $p$ vinylene polymeric backbones, which allows polymer flexibility and thermal stability to increase, while maintaining electronic delocalisation. ${ }^{39}$

The final polysiloles and silafluorene-containing polymers presented high quantum yields and excellent detection limits for explosive materials. The good sensitivity of polysiloles and silafluorene-containing polymers arises from the orbital energy matching between the excited-state ${ }^{1}$ SOMO (singlet singly occupied excited donor molecular orbital) of the polymeric materials and the LUMO of the explosive 
molecule. Another important factor is the silacycle moiety's (Lewis acid) ability to bind with the oxygen atoms in the nitro groups of explosive molecules. This interaction leads to an efficient photo-induced electron transfer from the polymer donor to the electronaccepting explosive.

A comparative study with the organic pentiptycene-containing polymers described in the previous section (see above) indicates that polysiloles and, in general, polymetalloles or metallole-copolymers, exhibit better quenching efficiencies than the former ones in toluene solution. ${ }^{22}$ However, organic pentiptycene-derived polymers offer superior sensitivity as solid-state sensors because of their higher molecular weight and more efficient energy migration in the solid state.

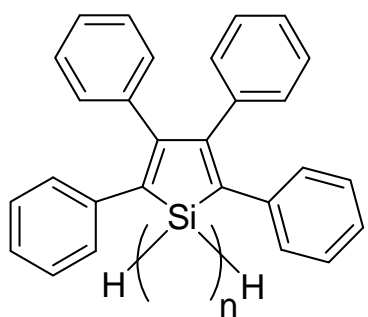

27
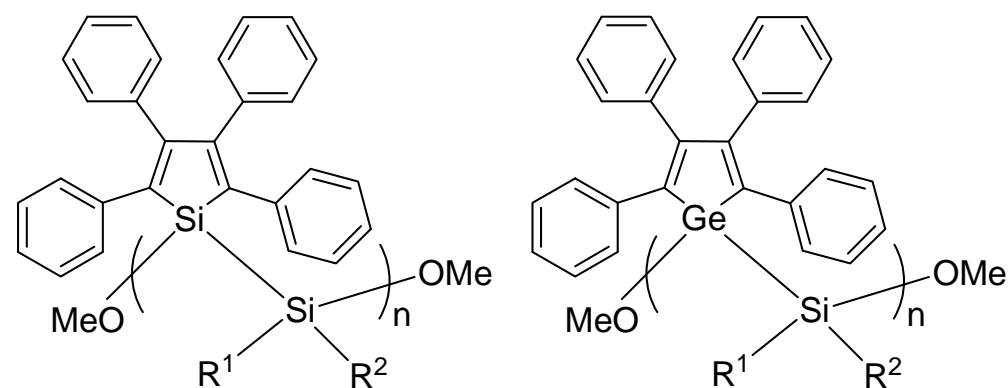

$$
\begin{array}{lll}
28 & R^{1}=H ; R^{2}=M e & 32 \\
29 & R^{1}=H ; R^{2}=P h & 33 \\
30 & R^{1}=P h ; R^{2}=M e & 34 \\
31 & R^{1}=H ; R^{2}=H & 35
\end{array}
$$

Fig. 13 Compounds 27-35

Trogler and col. reported the use of polytetraphenylsilole 27 (Fig. 13) as a suitable sensing polymer for TNT detection by fluorescence quenching. ${ }^{40}$ This inorganic polymer is stable to air, water, acids, common organic solvents and even seawatercontaining bioorganisms as the most common interferents. The colloidal nanoparticles of 27 in $\mathrm{THF} / \mathrm{H}_{2} \mathrm{O}$ suspensions were also prepared. ${ }^{41}$ They were obtained by adding water (in different percentages) to the THF solutions of polymer 27. The volumefractions between $80-90 \%$ of water induced the precipitation of highly fluorescent nanoparticles with a diameter of ca. $80 \mathrm{~nm}$. Addition of TNT to the suspensions of nanoparticles induced an impressive quenching of the emission intensity (quenching efficiencies improved by more than $400 \%$ for nanoparticles in relation to the dissolved oligosilole). Under these conditions, a detection limit below 20 ppb for TNT was obtained. 
The same group synthesised polymers and co-polymers 28-35 containing tetraphenylsilole or tetraphenylgermole with $\mathrm{Si}-\mathrm{Si}, \mathrm{Ge}-\mathrm{Ge}$ and $\mathrm{Si}-\mathrm{Ge}$ backbones. $^{42}$ Using these systems, detection of nitroaromatic molecules such as NB, 2,4-DNT, TNT and PA were explored. Upon the excitation of the toluene solutions of polymers 28-35 at $340 \mathrm{~nm}$, intense emission bands in the $480-510 \mathrm{~nm}$ range were observed. Addition of NB, 2,4-DNT, TNT and PA to the toluene solutions of polymers brought about different quenching degrees. Quenching efficiency followed the order of PA $>$ TNT $>2,4-\mathrm{DNT}>$ $\mathrm{NB}$, which is clearly related with the electron acceptor character of nitroaromatic molecules. Each polymer's emission quenching was different in the presence of the same nitroaromatic derivative, and the deactivation degree of a given polymer also differed depending on the explosives tested. In addition, these polymers were robust and insensitive to common interferents, such as organic solvents and inorganic acids. Finally, the authors suggested their possible use in sensing arrays to identify nitrated explosives by pattern recognition procedures.

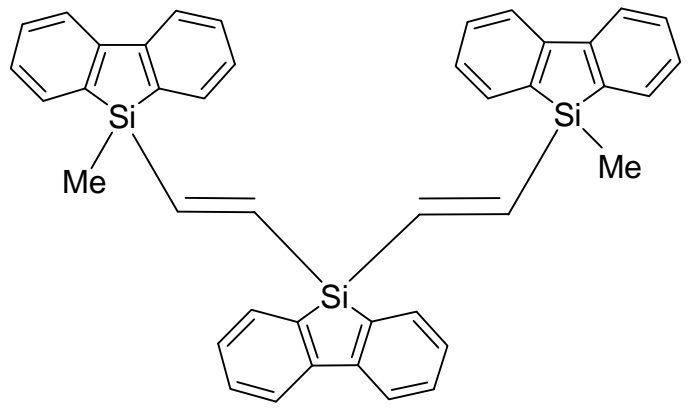

36<smiles>CC(C)(P)/C=C/[Si]1(C(C)(C)C)C(c2ccccc2)=C(c2ccccc2)C(c2ccccc2)=C1c1ccccc1</smiles>

38

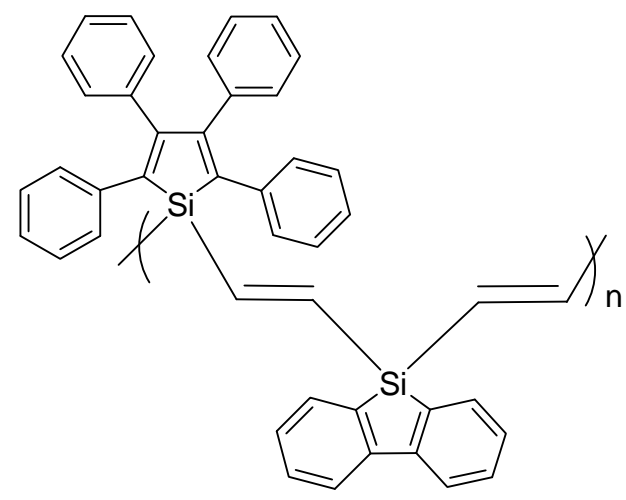

37

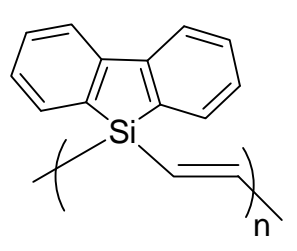

39

Fig. 14 Compounds 36-39

Trogler et al also evaluated the spectroscopic response of polymers 37-39 (Fig. 14) in the presence of nitrated explosives. ${ }^{43}$ The toluene solutions of polymers 37 and 38 
presented two intense absorption bands at ca. $300 \mathrm{~nm}$ and $390 \mathrm{~nm}$, whereas polymer 39 showed only one band at $295 \mathrm{~nm}$. Upon excitation, the toluene solutions of polymers 37 and 38 revealed intense emission bands at $493 \mathrm{~nm}$ and $492 \mathrm{~nm}$ for 37 and 38, respectively. For the toluene solutions of polymer 39, an intense emission band at 362 $\mathrm{nm}$ was noted. The emission bands of the three polymers were quenched to different extents upon the addition of nitroaromaric explosives TNT, 2,6-DNT and PA to the toluene solutions containing 37-39, whereas addition of RDX induced negligible changes. Quenching efficiency followed the order of PA $>$ TNT $>2,6-\mathrm{DNT} .{ }^{29} \mathrm{Si}-\mathrm{NMR}$ measurements of the interaction mode between trimer 36 (the model compound of polymer 39) and TNT were carried out. They indicated a Lewis acid-base interaction between the nitro groups of the nitroaromatic explosive and the silicon centres which facilitated an electron transfer process, yielding the quenching observed. Finally, and in order to test the possibility of applying these polymers in the detection of solid explosives, the response of the thin films of polymers 37-39 was analysed in the presence of TNT, 2,6-DNT, PA, RDX, HMX, Tetryl, TNG and PETN in the solid state. Polymers 37 and 38 exhibited emission quenching only in the presence of nitroaromatic explosives, whereas the emission of polymer 39 was quenched in the presence of all the explosives tested (with a remarkable detection limit of $200 \mathrm{pg} \mathrm{cm}^{-2}$ for Tetryl).
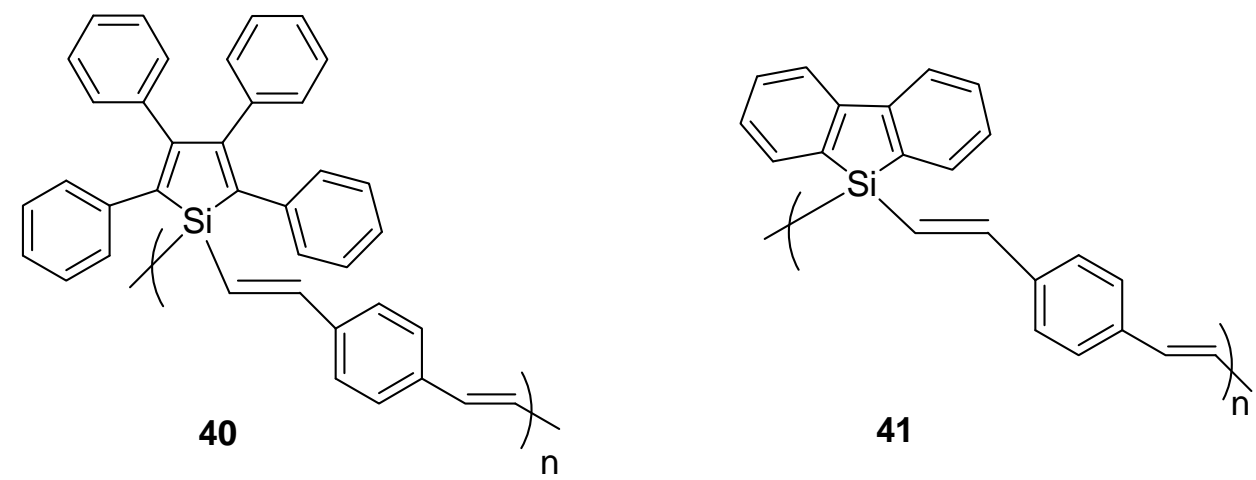

Fig. 15 Compounds 40 and 41

In another work, the same authors developed phenylene-divinylene-conjugated polymers 40 and 41 (Fig. 15) to test the influence of the phenylene aromatic ring in the detection of explosives. ${ }^{44}$ Polymers $\mathbf{4 0}$ and $\mathbf{4 1}$ were used for the fluorescent signalling of TNT, Tetryl, 2,4-DNT, PA, PETN, RDX, TNG and HMX in the solid state. Thin films showed intense emission bands which centred at 478 and $447 \mathrm{~nm}$ for $\mathbf{4 0}$ and $\mathbf{4 1}$, respectively. The emission of the thin films of $\mathbf{4 0}$ was quenched in the presence of the 
solid particles of nitroaromatic explosives (TNT, Tetryl, 2,4-DNT and PA) located on different surfaces (porcelain and filter paper), whereas the fluorescence of the films containing 41 was quenched in the presence of all the tested explosives. Neither polymer showed interference when films were exposed to common organic solvents (THF, benzene, toluene, acetonitrile, acetone and methanol), but gave some response in the presence of benzophenone and benzoquinone (oxidising aromatic molecules).

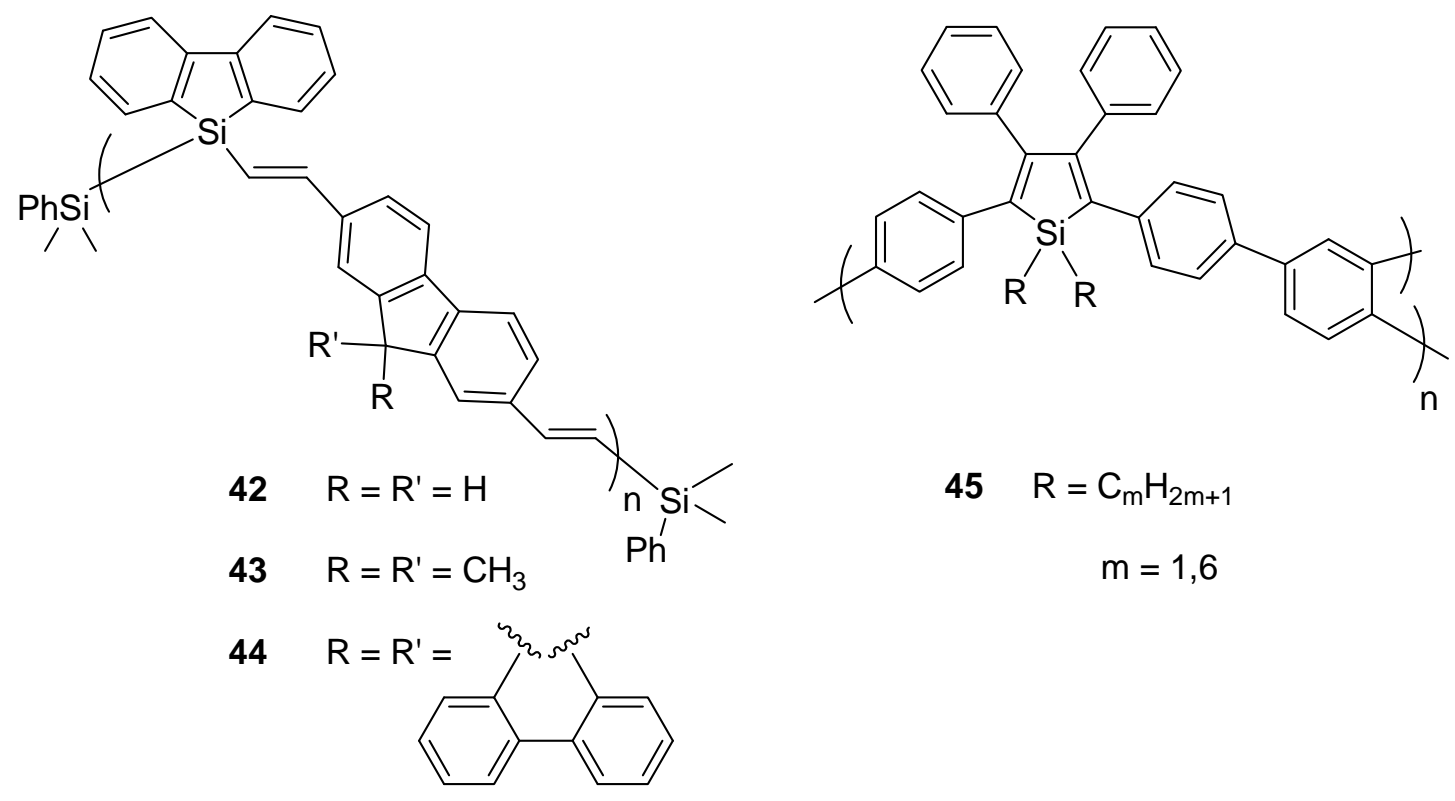

Fig. 16 Compounds 42, 43, 44 and 45

Trogler and Sanchez reported polymers 42-44 (Fig. 16) containing fluorenyl units anchored on the silicon centre of the silafluorene moiety via vinylene groups. ${ }^{45}$ Polymers contain trans-only frameworks with molecular weights in the range of 1300020000. Upon excitation of the toluene solutions of 42-44 at $345 \mathrm{~nm}$, intense emission bands were observed at ca. $376 \mathrm{~nm}$ for $\mathbf{4 2}$ and $\mathbf{4 3}$ and at 392 for $\mathbf{4 4}$. The thin films of these polymers were tested for the fluorescent detection of TNT, Tetryl, 2,4-DNT, PA, PETN, RDX, TNG and HMX in the solid state. As observed in other related polymers, the emission of 42-44 was quenched to a different extent in the presence of solid explosives. Lower detection limits were obtained for Tetryl $\left(1 \mathrm{pg} \mathrm{cm}^{-2}\right)$ using the thin films of polymer 42. Additionally, polymer 42 produced a turn-on fluorescence mechanism in the presence of PETN. Addition of PETN to the thin films of $\mathbf{4 2}$ induced initial emission quenching, but continued exposure to UV-light promoted the 
photochemical oxidation of fluorenyl moieties, which yielded a luminescent green fluorenone copolymer.

Very recently, Tang et al prepared hyperbranched poly(2,5-silole)s, which are closely related with the polymers described above. ${ }^{46}$ The THF solutions of polymer 45 produced an absorption band centred at ca. $379 \mathrm{~nm}$, and weak emission bands at 507 and $509 \mathrm{~nm}$ for $\mathrm{m}=1$ and $\mathrm{m}=6$, respectively (excitation at $373 \mathrm{~nm}$ ) due to the presence of active intramolecular rotations of the phenyl rings of the tetraphenylethene units that effectively deactivated the excited state of the polymer through rotational energy relaxation channels. However, addition of water to the THF solutions of $\mathbf{4 5}$ (up to 90\%) induced the formation of highly fluorescent aggregates. These emission enhancements were ascribed to an inhibition of the free rotation of the phenyl rings upon aggregation. Emission intensity was quenched with the gradual addition of PA to the THF-water 1:9 $\mathrm{v} / \mathrm{v}$ suspensions of the aggregated nanoparticles. The detection limit of PA using $\mathbf{4 5}$ was $1 \mathrm{ppm}$. The fact that the aggregated polymer presented internal cavities, plus the Lewis acid-base interactions between the silole moieties and the nitro groups of PA, favoured an efficient photo-induced electron transfer accounting for the observed quenching.

Apart from the use of silole- and silafluorene-containing systems, other Sicontaining polymers have been reported for the detection of explosives. For instance, Cheuk et al recently published the hyperbranched poly(silylenevinylene) polymer 46 (Fig. 17). ${ }^{47}$ The THF solutions of $\mathbf{4 6}$ presented a weak emission band at $500 \mathrm{~nm}$ which, like the case cited above, was enhanced in the presence of water due to polymer aggregation. The sensing behaviour of polymer $\mathbf{4 6}$ resembled that obtained for $\mathbf{4 5}$, and the addition of PA to the THF-water 1:9 v/v suspensions of the aggregated nanoparticles of 46 induced immediate quenching. The detection limit measured for PA was about 1 ppm. 


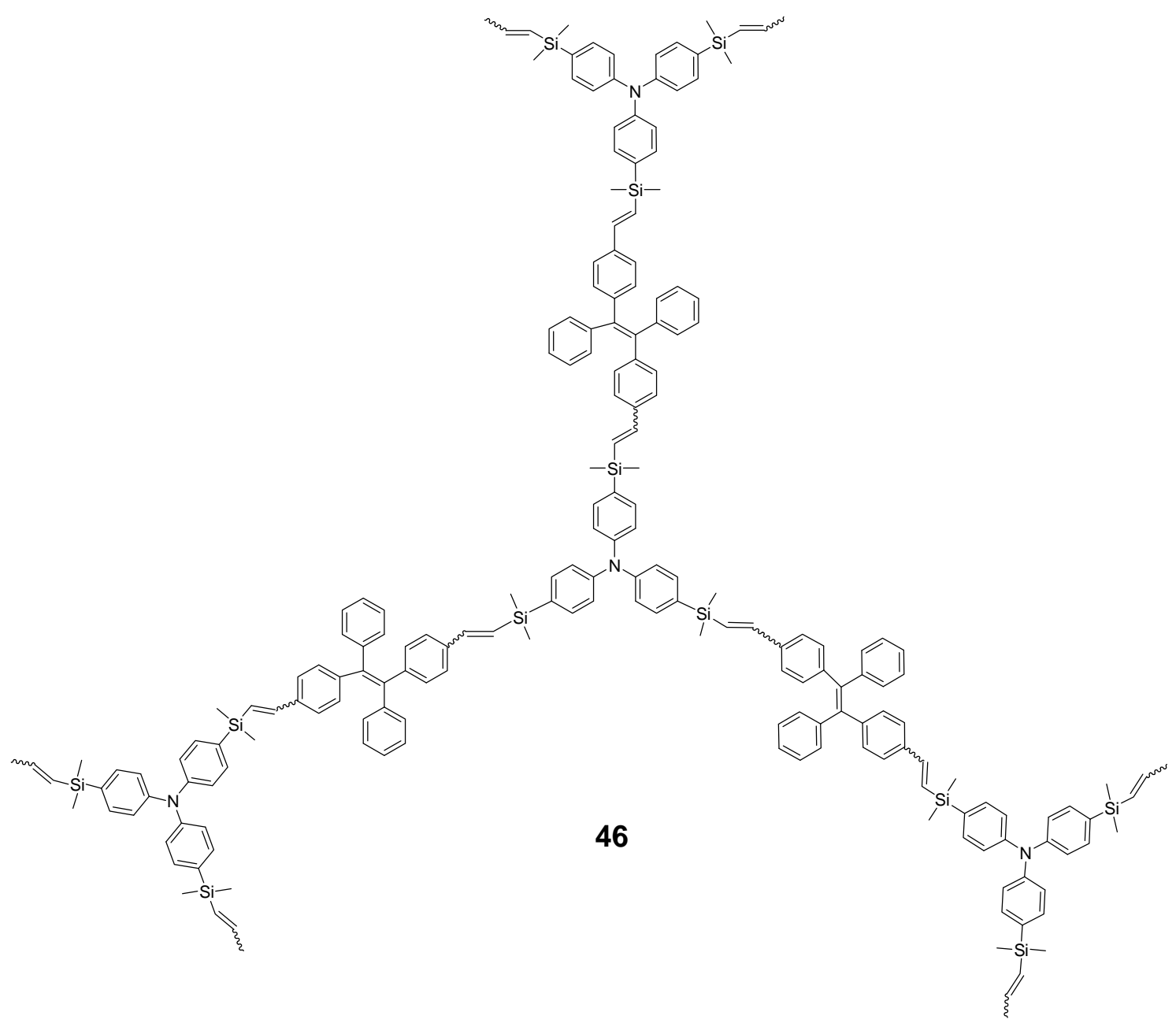

Fig. 17 Compound 46<smiles>CC(C)=C(C)C(C)C(=C(C)C)c1ccc(S(C)(=O)=O)cc1</smiles>

47

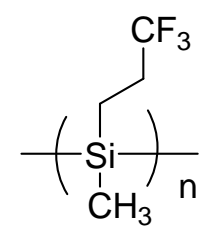

48

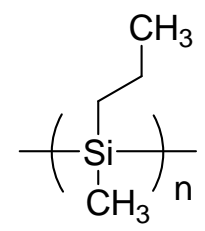

49

Fig. 18 Compounds 47, 48 and 49 
Schanze et al prepared fluorescent thin films using polymer poly[1-phenyl-2-(4trimethyl)sililphenyl]ethyne 47 (Fig. 18). ${ }^{48}$ This polymer was highly permeable to light gases (i.e., $\mathrm{N}_{2}, \mathrm{O}_{2}, \mathrm{H}_{2}$ ) and hydrocarbon vapours. ${ }^{49}$ This high permeability was ascribed to the polymers' large fractional free volume and to the presence of interconnected channels that allow small molecules to rapidly diffuse within the matrix. These authors observed that the fluorescence of polymer $\mathbf{4 7}$ was strongly quenched by vapours from a variety of nitroaromatic compounds (1,4-DNB, 1,3-DNB, 2,6-DNT, 2,4-DNT, 4-NT and NB), present at levels ranging from ppm to ppb in air. As with other polymers, quenching is believed to arise from the formation of charge-transfer complexes between electron-poor nitroaromatic quenchers and electron-rich 47.

Fujiki et al prepared polymers $\mathbf{4 8}$ and $\mathbf{4 9}$ and tested their fluorescent behaviour in the presence of nitroaromatic explosives. ${ }^{50}$ The THF solutions of $\mathbf{4 8}$ showed an intense emission band at $335 \mathrm{~nm}$ which underwent severe quenching in the presence of PA, TNB, 2,4-DNT and 1,3-DNB. Greater quenching was noted for PA and TNB. In spite of these interesting results, the fluorescence of polymer 49 remained unchanged in the presence of nitroaromatic explosives. This fact could be ascribed to the electronwithdrawing character of the $-\mathrm{CF}_{3}$ groups in 48. These groups increase the positive charge on the silicon atom in the backbone facilitating the interaction between this atom and the nitro groups of explosives. This enhanced interaction for 48, if compared with 49, enabled an efficient photo-induced electron transfer, which was the main factor of the observed quenching. The thin films of polymer $\mathbf{4 8}$ were also used for the signalling of PA and 2,4-DNT in water. One of the advantages of the thin films of $\mathbf{4 8}$ was the recovery of the sensory material by rinsing with water or methanol.

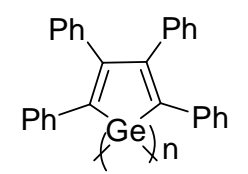

50

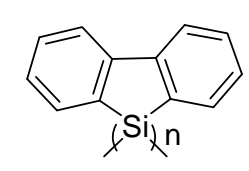

51

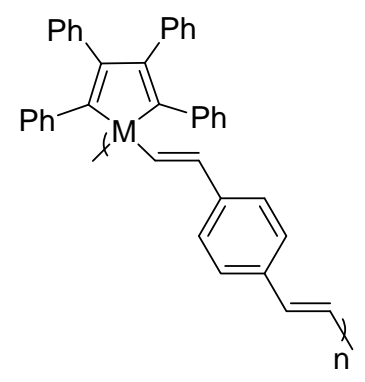

52

Fig. 19 Compounds 50, 51 and 52 
A visual detection of trace residues of nitroaromatic explosives (TNT, 2,4-DNT and PA) on surfaces using the thin films of photoluminescent metallole-containing polymers $(\mathbf{2 7}, \mathbf{4 0}, \mathbf{4 1}, \mathbf{5 0 - 5 2})$ has been achieved (Fig. 19). ${ }^{51}$ In relation to this, the different polymers showed intense emission bands $(510 \mathrm{~nm}$ for $\mathbf{2 7}, 500 \mathrm{~nm}$ for $\mathbf{5 0}, 495$ $\mathrm{nm}$ for 40 and $475 \mathrm{~nm}$ for $52(\mathrm{M}=\mathrm{Ge}$ or $\mathrm{Si})$ and $360 \mathrm{~nm}$ for 51 and $\mathbf{4 1})$ upon excitation at $340 \mathrm{~nm}$. Emissions of the different films were quenched to different degrees in the presence of TNT, DNT and PA. These polymeric films were successfully employed for the naked eye detection of TNT, 2,4-DNT and PA on certain surfaces (porcelain and paper).

\subsection{4.- Molecularly imprinted polymers (MIPs)}

The molecular imprinting technique allows the formation of specific recognition sites in macromolecules. In this process, functional and cross-linking monomers are copolymerised in the presence of a target analyte (template). Functional monomers form a complex with the imprinting molecule, and the functional groups are held in position by highly cross-linked structures in the subsequent polymerisation. Subsequent removal of the template reveals binding sites that are complementary in size and shape to the analyte. Although most MIP applications have been limited to the separation of mixtures, MIP-based sensing systems have also been developed. In the particular case of the detection of explosives, several interesting examples have been reported to date.

For instance, S.A. Grant et al. ${ }^{52}$ designed MIP-based sensors that utilised quantum dots as fluorescent antennas for detecting TNT. These authors employed a methacrylic acidbased polymer containing a methyl group and a carboxylic acid moiety as side groups. Using the carboxylic acid functional group, MIPs were labelled with aminefunctionalised quantum dots. The templates used for the synthesis of MIPs were 2,4DNT and TNT. Among the solvents used in the synthesis (acetonitrile, chloroform, toluene and DMF), chloroform gave rise to the imprinting polymer which decreased the most in fluorescence after treatment with the analyte. Addition of $0.5 \mathrm{mM}$ of 2,4-DNT to the aqueous solutions of the MIP-2,4-DNT sensor induced an immediate drop in fluorescence intensity, with an exponential trend over time. When TNT was used as a template, the addition of this analyte to an aqueous solution containing the MIP sensor gave rise to a similar behaviour to that observed with 2,4-DNT. Detection limits were $30.1 \mu \mathrm{M}$ for 2,4-DNT and $40.7 \mu \mathrm{M}$ for TNT. MIPs' sensing behaviour contrasted with 
that observed for the non-imprinting polymer, which displayed a poor or negligible response in the presence of these nitroaromatic derivatives.

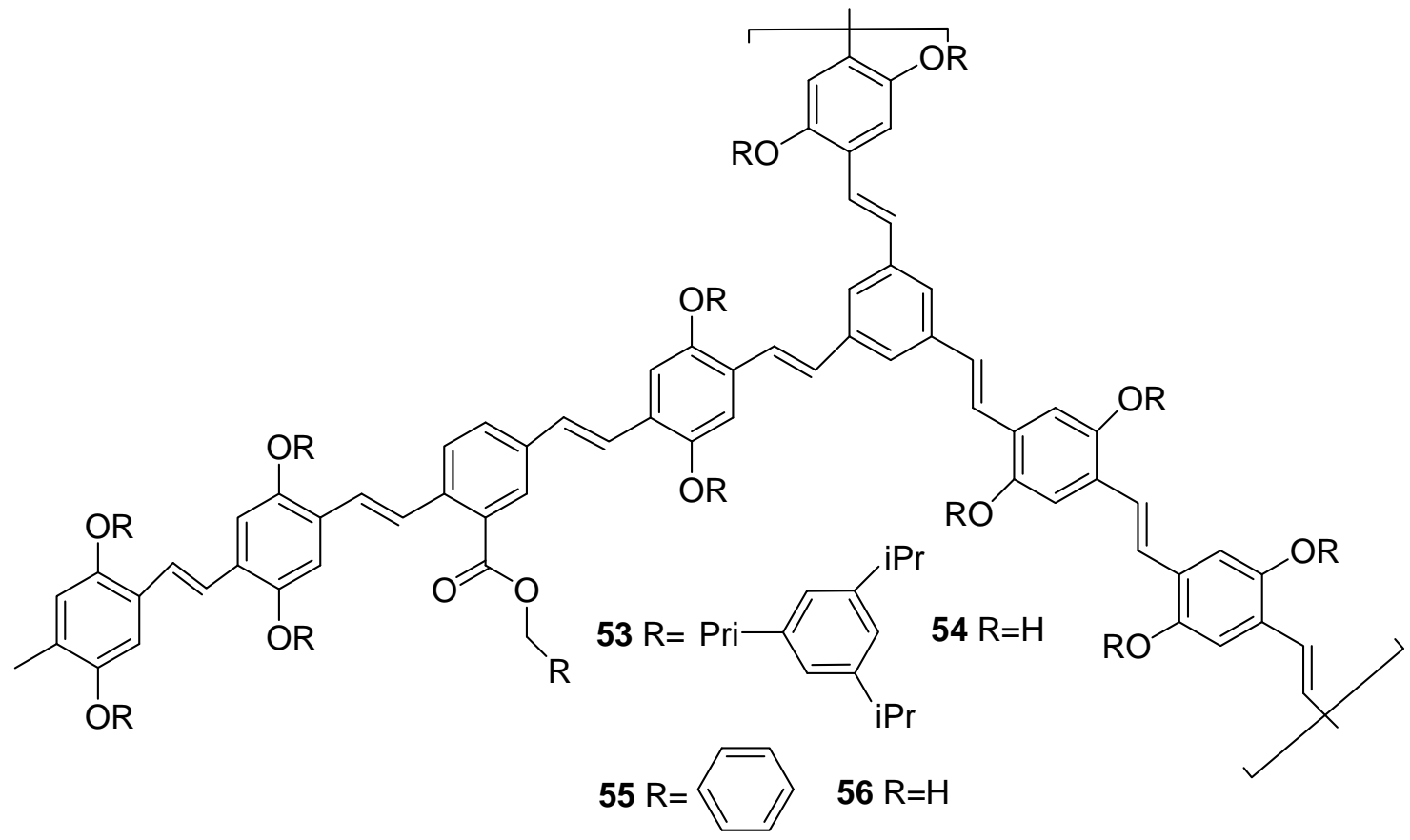

Fig. 20 Compounds 53, 54, 55 and 56

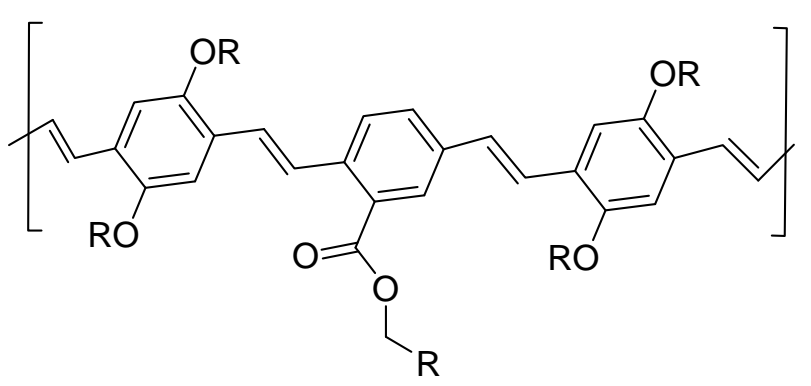<smiles>CC(C)c1cc([Pb]=[SbH])cc(C(C)C)c1</smiles>

Fig. 21 Compounds 57 and 58

As stated in previous sections, conjugated polymers-based sensor devices usually show higher sensitivity than small molecule-based chemosensors due to conjugated polymers' intrinsic ability to provide an amplified response to the analyte-binding event. E. E. Nesterov et al. ${ }^{53}$ described the use of molecularly imprinting polymers using conjugated polymers (MICPs) for the detection of explosives in which the conjugated polymer is used as both imprinting materials and analytical signal transductors. Threedimensionally cross-linked poly(p-phenylene vinylene)s were used as they show some degree of conformational flexibility, required for shaping the imprinting cavity. In addition, poly( $p$-phenylene vinylene)s are highly emissive in the solid state and their 
fluorescent properties are strongly affected by the presence of quenchers. The imprinting cavity was formed between separate poly(p-phenylene vinylene) strands which were held rigidly together by cross-linking units. To avoid problems deriving from the difficulty of template removal, which could give rise to high initial fluorescence quenching, these authors chose 2,4,6-triisopropyltoluene as a nonquenching template to prepare a "TNT imprinting" polymer given the similarity in molecular shape between 2,4,6-triisopropyltoluene and TNT. Following this approach, polymer 53 (Fig. 20) was isolated as non-soluble fluorescent spherically shaped micrometer-sized particles. For comparative studies, both polymers 55 and 57 (Fig. 21) were prepared. 57 was a linear polymer prepared in the absence of the crosslinker, and was soluble in several organic solvents. On the other hand, polymer 55 was prepared under the same conditions used for 53, but on this occasion, the template used was toluene. The intention was to know the real effect of the imprinting mechanism. Removal of the template gave rise to polymers 54, 56 and 58. The degree of template removal was around $50 \%$ for ligand $\mathbf{5 3}$, and was practically quantitative for ligands 55 and 57.

Exposure of these polymers to saturated TNT vapours at room temperature resulted in noticeable fluorescence quenching for them all. As expected, polymer 54 showed better sensitivity to TNT than its non-imprinting linear analogue 58, or template-containing precursors 53, 55 and 57. Polymer 56 imprinted with the wrong template also displayed lesser sensitivity to TNT. One remarkable and clearly unexpected observation was that exposure of $\mathbf{5 4}$ to the vapours of 2,4-DNT caused much stronger fluorescence quenching than TNT. Furthermore, polymer $\mathbf{5 3}$ was slightly affected only by 2,4-DNT. Exposure of polymer 54 to 2,4-DNT or TNT vapours for 10 minutes caused a substantial decrease in fluorescence intensity (which was almost complete for 2,4-DNT). In addition, the fluorescence of the samples markedly recovered after maintaining them for 10 minutes in air, and fluorescence recovered completely in 1 h. This exposure to quenchers with the subsequent recovery of fluorescence can be repeatedly done with no noticeable irreversible quenching. In contrast, linear polymer 58 became irreversibly quenched upon exposure to 2,4-DNT or TNT.

In addition to fluorescent MIP probes, mass sensitive devices (such as quartz crystal microbalances) coated with MIPs have been suggested to provide promising low-cost detection systems for nitroaromatic explosives in the vapour phase. ${ }^{54}$ 


\subsection{5.- Miscellaneous}

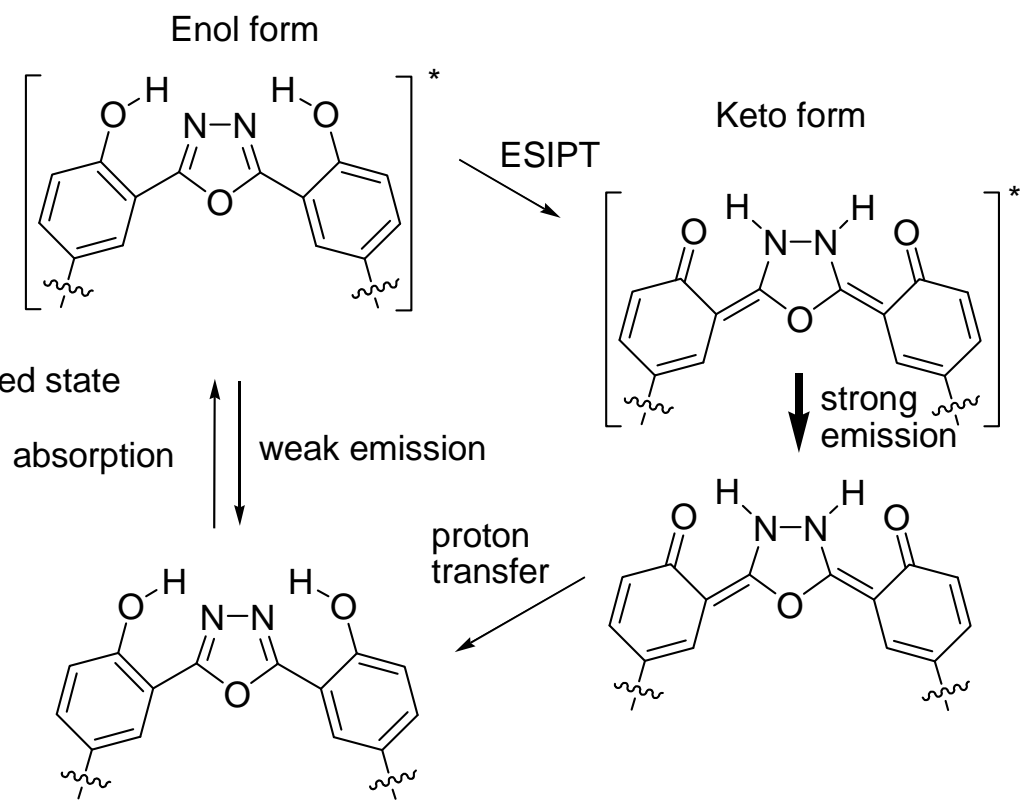

Ground state

Fig. 22 Scheme of an ESPIT process that is active in oxadiazoles functionalised with $o$ hydroxyphenyl groups.

Organic fluorescent conjugated polymers with nitrogen-containing fused-ring systems have recently received much attention because they usually show high fluorescence. ${ }^{55}$ In particular, oxadiazole-based materials have been reported to display a considerable Stokes shift and can be easily prepared with tailor-made structural modifications. Additionally, it has been established that the incorporation of an $O$ hydroxyphenyl group into the 2-position of oxadiazole provides an excited-state intramolecular proton transfer (ESIPT) process involving the tautomeric enol and keto forms (Fig. 22). ESIPT provides two emission bands from the enol and keto structures; the keto form is responsible for longer wavelength emission. 

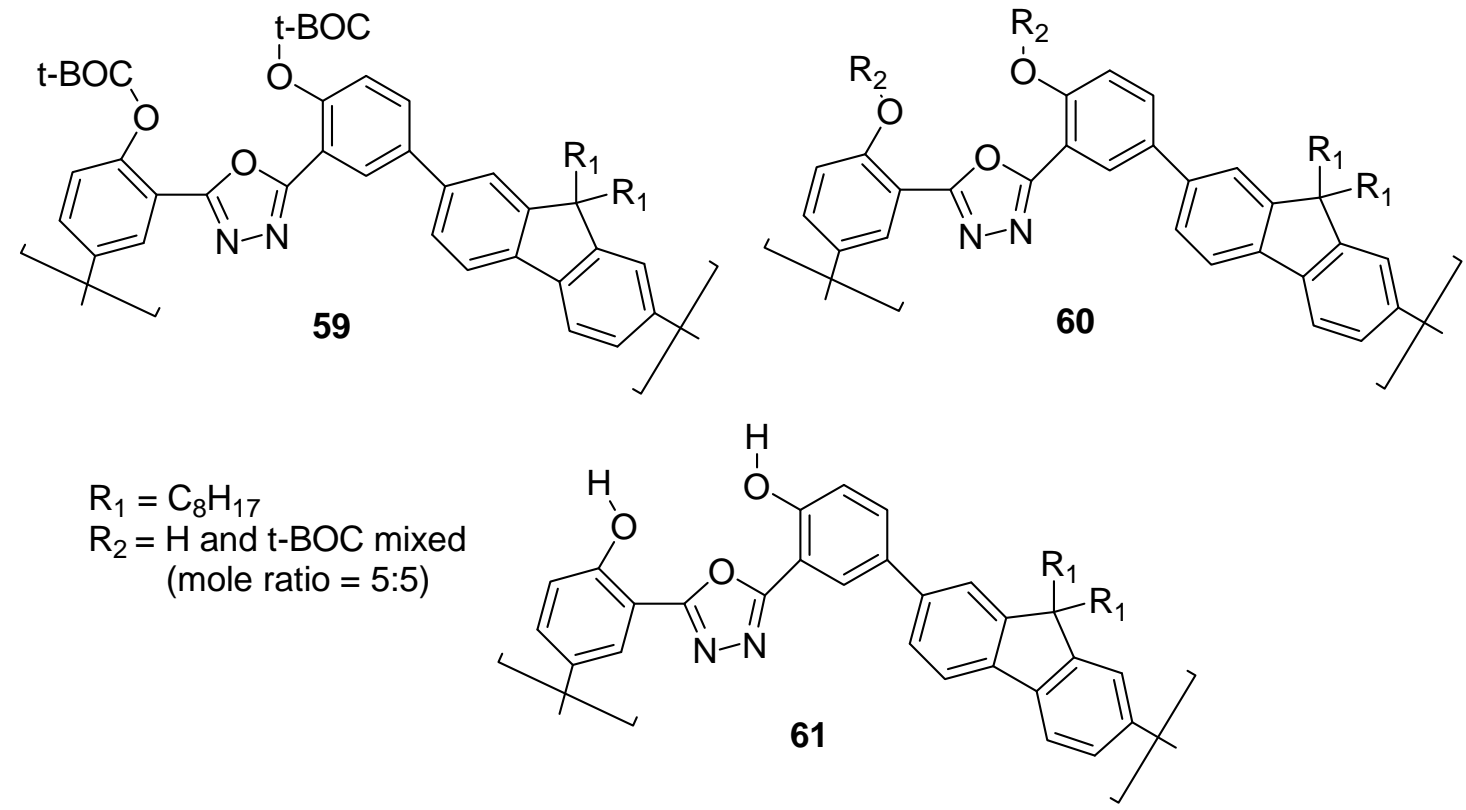

Fig. 23 Compounds 59, 60 and 61

Based on these ideas, T. S. Lee et al. prepared oxadiazole-based fluorescent conjugated polymers 59-61 (Fig. 23) and investigated their chemosensing properties to electron-deficient nitroaromatic derivatives. ${ }^{56}$ All three polymers presented a low energy absorption band at $330 \mathrm{~nm}$ in chloroform. Additionally, a long wavelength emission at $528 \mathrm{~nm}$ was found for $\mathbf{6 0}$ and $\mathbf{6 1}$ which resulted from ESIPT. Polymer $\mathbf{5 9}$ did not exhibit the keto form emission because of the effective blocking of proton transfer processes by the t-BOC group. Apart from the strong emission at $528 \mathrm{~nm}$, a significantly weak emission at a short wavelength of around $410 \mathrm{~nm}$ was also observed in 60 and 61, which results from enol form relaxation. The solutions of polymer 60 in chloroform were titrated with nitroaromatic derivatives such as PA, 2,4-DNT and 1,3DNB. A dramatic quenching of the emission behaviour of $\mathbf{6 0}$ was noted in these experiments. Concomitantly, absorption of the polymer presented a hyperchromic effect upon exposure to PA and DMB without a red shift or a blue shift. This effect is presumably the result of an electron transfer occurring within the electron-deficient nitroaromatic compounds and the bis(hydroxyphenyl)oxadiazole moiety. These studies indicated that PA was the most efficient quencher, followed by 1,3-DNB and 2,4-DNT. 


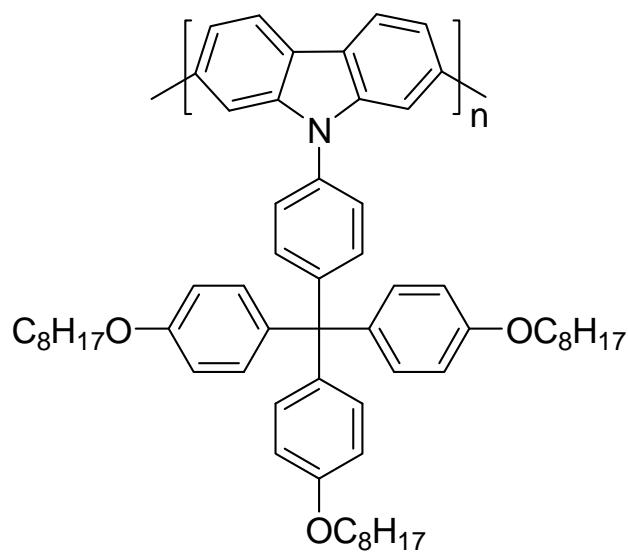

62

Fig. 24 Compound 62

Zhang et al. reported the use of conjugated carbazole polymer 62 (Fig. 24)as an efficient sensory material for the detection of TNT and 2,4-DNT traces. ${ }^{57}$ The presence of the bulky side chain in the structure not only prevents the $\pi$-staking interaction between polymer chains, which contributes to high fluorescence efficiency, but also generates an efficient pathway and benefits the diffusion of explosives. Furthermore, the moiety of the side chain endows the polymer good solubility and helps form high quality films by spin-coating. The absorption spectrum of the films of $\mathbf{6 2}$ showed two maxima at 270 and $395 \mathrm{~nm}$. In the fluorescence spectrum, 62 showed a blue emission at $417 \mathrm{~nm}$. The time-dependent fluorescence intensity of the film of $62(7 \mathrm{~nm})$ upon exposure to TNT vapours revealed a reduction of $57.4 \%$ in $30 \mathrm{~s}$ and of $73.0 \%$ in $60 \mathrm{~s}$. This suitable performance resulted from carbazole's good donor ability and the film's non-compact structure. In addition, 62 displayed good selectivity to TNT if compared to some potential interfering gases such as $\mathrm{HCl}, \mathrm{NO}_{2}, \mathrm{SO}_{2}, \mathrm{CO}_{2}$ and alcohol. The films of 62 also gave a response to 2,4-DNT vapours, although the response was not as sensitive as it was for TNT. The authors suggested that this might be due to TNT's electron accepting capability, which is higher than that of 2,4-DNT. 


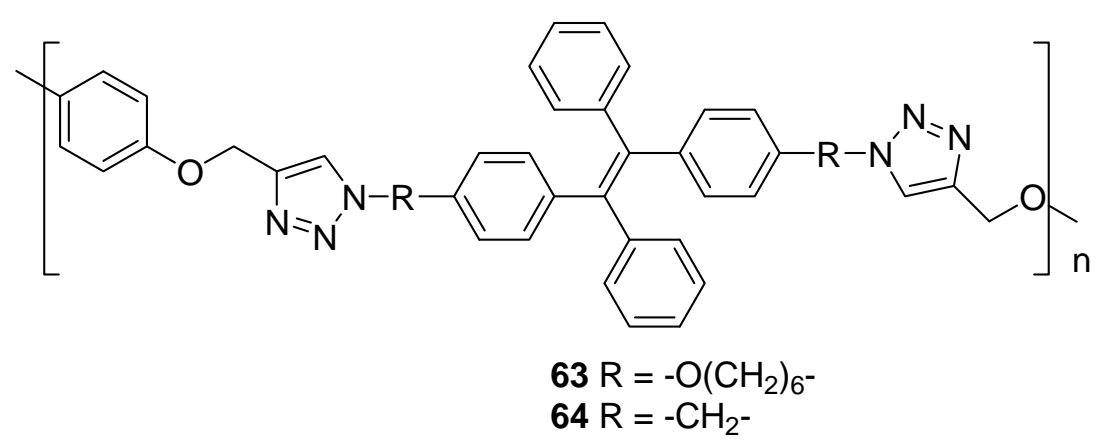

Fig. 25 Compounds 63 and 64

Tang et al. ${ }^{58}$ reported a family of new tetraphenylethene-containing polytriazoles in which light emission was dramatically enhanced by aggregate formation. For instance, polymers 63 and 64 (Fig. 25) were not emissive in THF solution, but strong emission bands were observed at around $480 \mathrm{~nm}$ after addition of water ( $>60 \mathrm{vol} \%$ and 10 vol \%, respectively). These results confirm that both polymers presented an aggregation-induced emission which is characteristic of tetraphenylethene derivatives. 63 and 64 in the THF/water and DMF/water mixtures were used as probes for the detection of explosives. In these studies, PA was used as a model. Addition of PA to solutions of $\mathbf{6 3}$ or $\mathbf{6 4}$ gave rise to fluorescence quenching at concentrations as low as 0.1 ppm. A lineal correlation was observed in the $10-30 \mu \mathrm{g} / \mathrm{mL}$ concentration range. The overlap between the bands of PA and the polymers in the wavelength region of 385-485 $\mathrm{nm}$ facilitated the energy transfer from the excited states of polymers to the ground state of PA, resulting in fluorescent quenching. These authors also adsorbed polymers on filter papers and demonstrated their use for the detection of PA in toluene solutions.<smiles>Cc1c(CCC(=O)OC(C)COCC(C)(CC(C)(O)CC(C)(C)C)c2ccccc2)c(=O)oc2c(C)c(OCC(=O)OC(C)(C)C)ccc12</smiles>

65<smiles>Cc1c(CCC(=O)NCCC[Si](C)(C)O[Si](C)(C)O[Si](C)(C)C)c(=O)oc2c(C)c(OCC(=O)OC(C)(C)C)ccc12</smiles>

66

Fig. 26 Compounds 65 and 66 
Kumar et al synthesised 65 and 66 (Fig. 26), which are co-polymers of 4,8dimethylcoumarins with poly(ethyleneglycol) (PEG) and poly(dimethylsiloxane) (PDMS), respectively. ${ }^{59}$ The chloroform solutions of polymer 65 showed an intense emission at $420 \mathrm{~nm}$, which was assigned to the coumarin fluorophore. The emission band was progressively quenched upon the addition of increasing amounts of TNT and 2,4-DNT in chloroform. The thin films of polymer 65 also displayed emission quenching in the presence of TNT and 2,4-DNT vapours (the quenching efficiency order was 2,4-DNT $>$ TNT). Practically the same response was observed for polymer 66 in both solution and vapour studies.

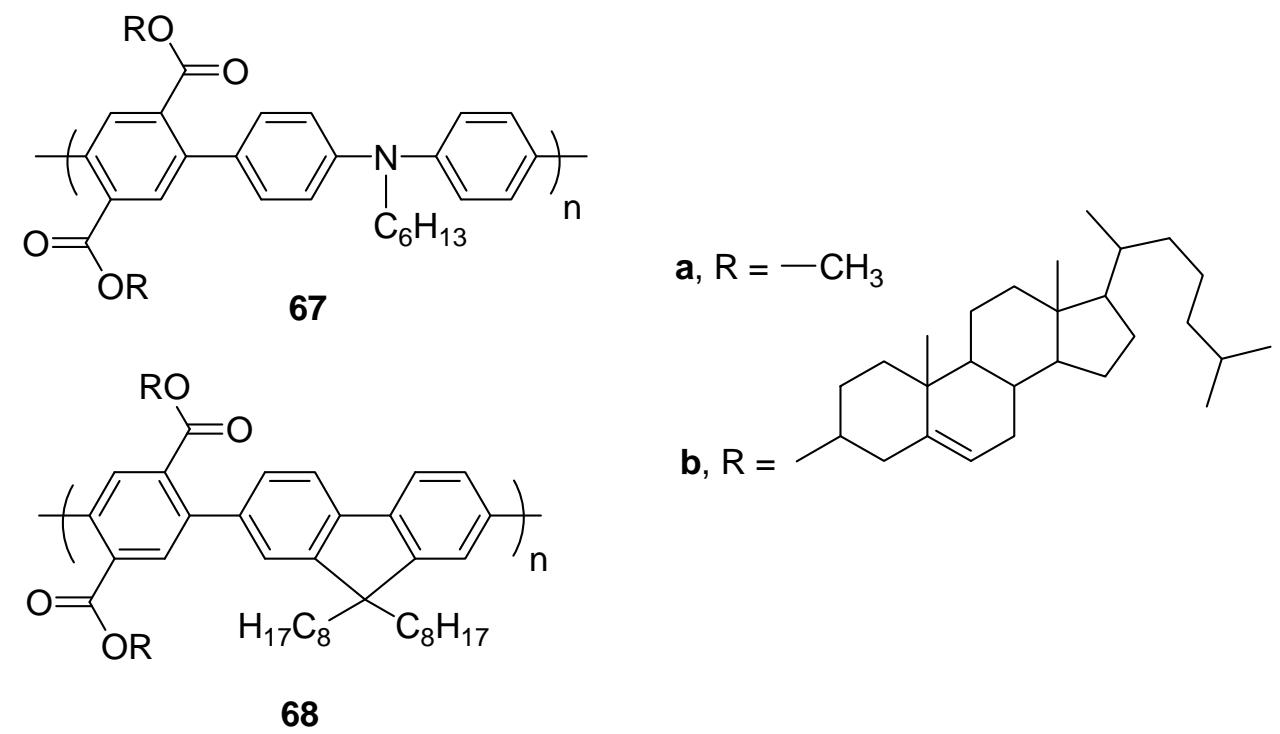

Fig. 27 Compounds 67 and 68

Polymers 67a, 67b, 68a and 68b (Fig. 27), coated on a glass substrate, showed intense emission bands at 532, 504, 451 and 438, respectively. Exposure of the four polymers to 2,4-DNT vapours induced different degrees of quenching. The performance of all these polymers mounted on the end tip of an optical fibre was also studied. The best results were obtained with the optical fibre containing polymer $\mathbf{6 8 b}$; quenching of $65 \%$ was observed after $1 \mathrm{~min}$ in the presence of 2,4-DNT vapours. ${ }^{60}$ 


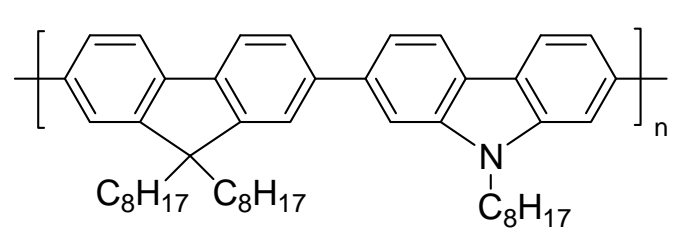

69

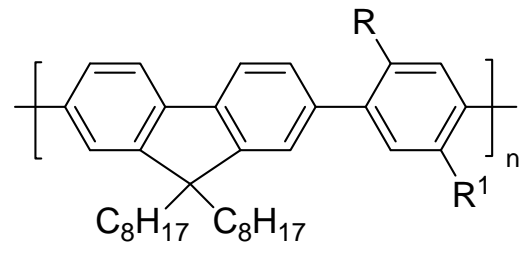

$70 \mathrm{R}=\mathrm{R}^{1}=\mathrm{OC}_{8} \mathrm{H}_{17}$

$71 \mathrm{R}=\mathrm{N}\left(n-\mathrm{C}_{8} \mathrm{H}_{17}\right)_{2} \mathrm{R}^{1}=\mathrm{H}$

$72 \mathrm{R}=\mathrm{R}^{1}=\mathrm{N}\left(\mathrm{n}-\mathrm{C}_{8} \mathrm{H}_{17}\right)_{2}$

Fig. 28 Compounds 69-72

The poly(phenylene)s 69-72 (Fig. 28) derivatives bearing fluorine moieties have been reported to display moderate quenching (20\% deactivation for 70 ) in the presence of DMNB vapours. This quenching was ascribed to a photo-induced electron transfer process from the excited fluorophore to the DMNB quencher. ${ }^{28}$

\section{2.- Hybrid materials}

Different approaches for the development of fluorogenic hybrid systems to detect explosives involving the use of silica microspheres, silica nanoparticles, films, fibres and silicon wafers have been described.

Walt and co-workers ${ }^{61}$ reported an optical microsensor for the high-speed detection of explosives in the vapour phase using the simple porous silica microsphere packing material from HPLC columns (3-5 $\mu \mathrm{m})$ as a support in which a fluorescent dye (red nile) was physisorbed. Differences in the excitation and emission spectra of the physisorbed dye were found with the different functionalisations (amino, cyano, butyl and hydroxyl groups) of the microspheres surface due to different polarity. Each microsphere was attached to the surface of a glass cover slip. Hence, each microsensor's individual response was recorded with a fluorescence microscope and a high performance CCD camera. In this way, the authors obtained images containing 1000 sensors which can be addressed individually. These authors also used microspheres as individual sensors positioned in the distal wells on the surface of an etched imaging fibre, which was utilised for remote sensing tasks. Vapour exposures to a single element sensor array revealed that the response profiles were highly reproducible from sensor to sensor and that the response profiles resulting for each analyte differed. One important advantage of employing high density sensor arrays is the ability to combine sensor 
responses in an averaged response, giving rise to signal-to-noise ratio enhancement. Incorporating many identical sensor responses allowed the detection of $23 \mathrm{ppb}$ of 2,4DNT and $80 \mathrm{ppb}$ of 1,3-DNB.

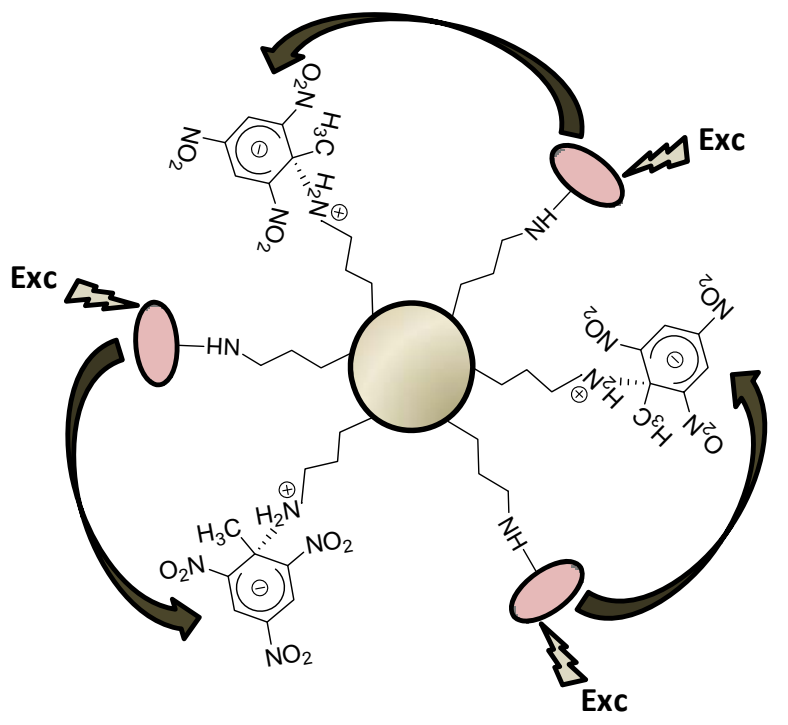

Fig. 29. FRET-based silica nanoparticles sensors for TNT detection

In a more recent paper, silica particles were also employed as a support for the ultrasensitive detection of TNT in both solution (ethanol/acetonitrile) and the vapour phase. ${ }^{62}$ On this occasion, a FRET-based mechanism was proposed as the signalling paradigm. The surface of nanoparticles was functionalised with both a fluorophore (6carboxy-rhodamine) and amines. Amines were able to bind TNT molecules, while the TNT-amine complex formed in close proximity to the fluorophore enhanced quenching of the fluorescence by a FRET mechanism (Fig. 29). The authors also used functionalised nanoparticles on a silicon wafer with etched microwells, and found that this format enabled a TNT detection as low as $1 \mathrm{nM}$ in solution. A better performance of this assembly was noted when compared with nanoparticles in solution. For the detection of TNT in the vapour phase, a piece of filter containing nanoparticles was used. The response to TNT vapours was higher than for other explosives such as 2,4DNT, NB or RDX. 


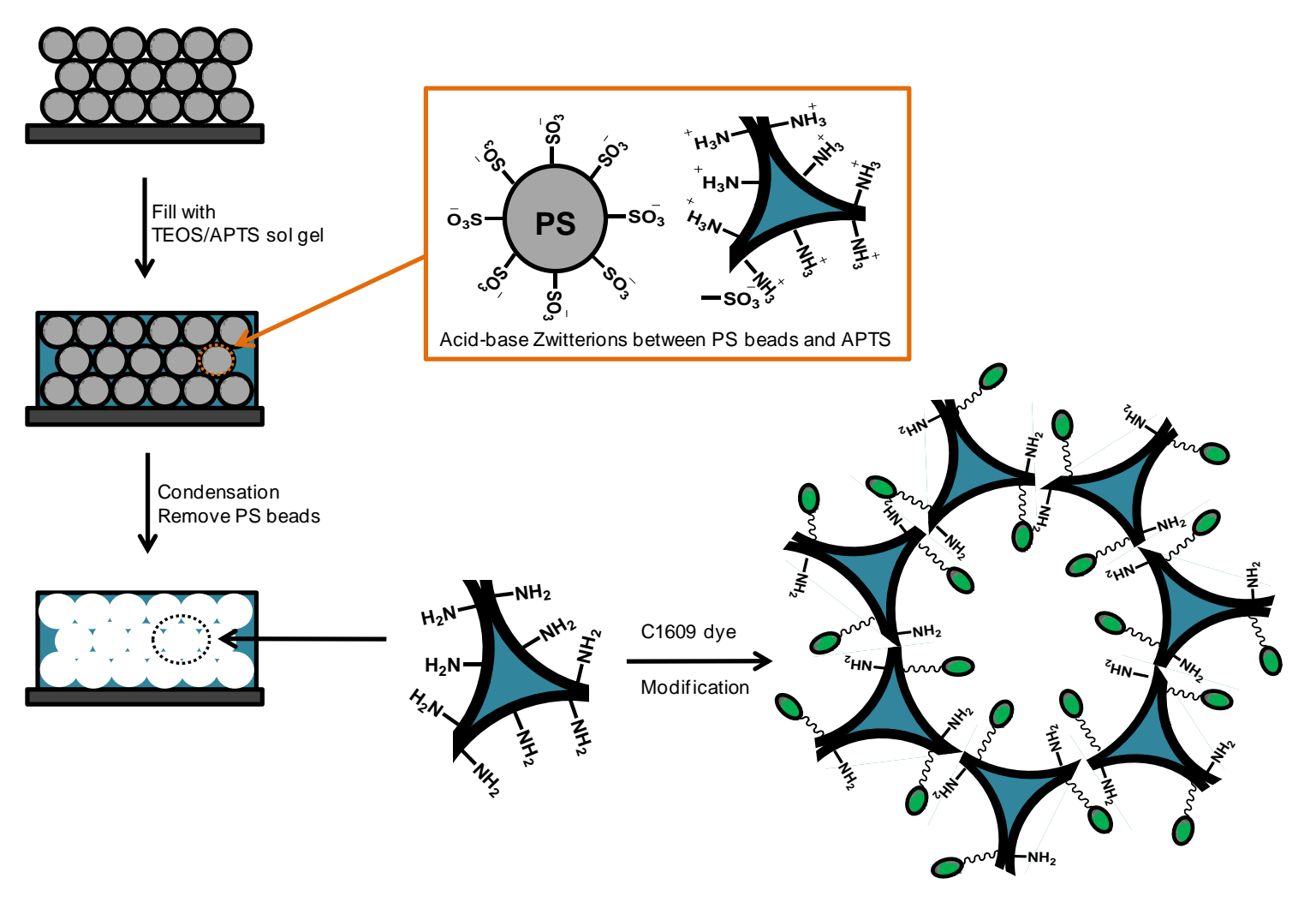

Fig. 30 Fabrication and modification with a dye of the inverted opal silica film containing a hybrid monolayer of amino groups.

A similar approach was used by Zhang et al. who developed a fluorescence chemosensor for the ultrasensitive detection of explosive nitroaromatic vapours by also using a FRET-based quenching mechanism. ${ }^{63}$ In this case, sensing material consisted in an inverted opal silica film containing a hybrid monolayer of amino groups and dye molecules (Fig. 30). The amino groups selectively captured TNT vapour molecules through the formation of TNT-amino complexes. Fluorescein 5(6)-carboxyfluoresceinN-hydroxysuccinimide ester (C1609 dye) was used as a signalling unit because its emission band completely overlapped the absorption band of the TNT-amino ensemble. The fluorescent response of the C1609-( $\left.\mathrm{NH}_{2}\right)$-silica inverted opal film to saturated nitroaromatic derivatives was NB $>2,4-\mathrm{DNT}>\mathrm{TNT}$. However, the quenching percentage for TNT was surprisingly higher than that expected for the relative vapour pressures and diffusion velocity of this analyte. 


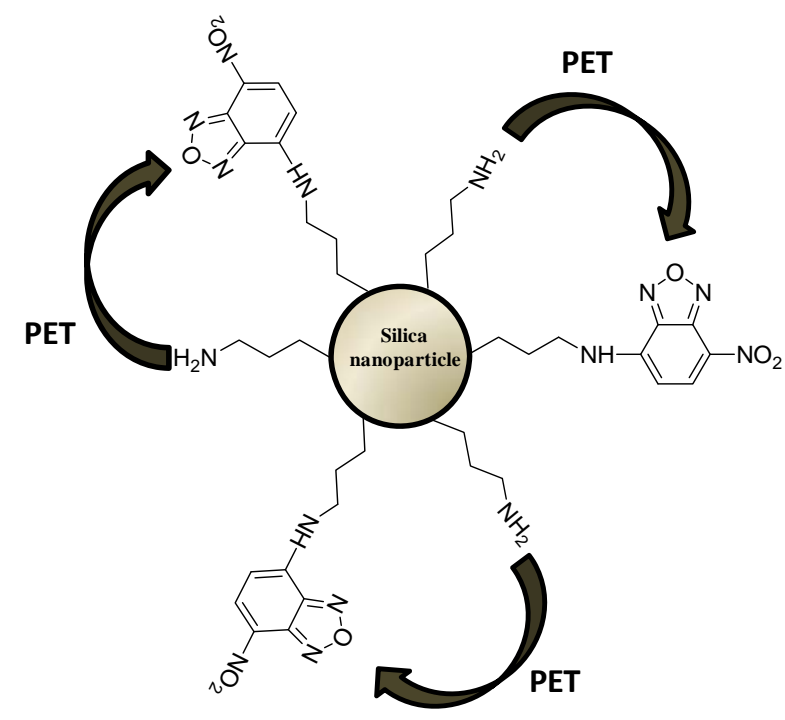

Fig. 31 Schematic illustration of NBD-( $\left.\mathrm{NH}_{2}\right)$-silica nanoparticles

The same group prepared silica nanoparticles functionalised with both free amines as receptors and nitrobenzoxadiazole (NBD) as a signalling unit. ${ }^{64}$ The NBD-( $\left.\mathrm{NH}_{2}\right)$ silica system displayed reversible and controllable emission changes in the presence of protons or TNT. Protons increased fluorescence intensity (fluorescence ON) when binding to the nitrogen atoms of the anchored amines. Inhibition of a PET process from the nitrogen to the excited NBD was proposed as the mechanism to explain emission enhancement (Fig. 31). However, when TNT binds anchored amines, fluorescence quenching was observed (fluorescence OFF) due to a FRET mechanism based on the strong charge-transfer interaction between the NBD and the Meisenheimer complex formed between TNT and amines. As regards the interaction with protons, fluorescence intensity increased with the addition of successive $\mathrm{HCl}$ aliquots to the system. This enhancement of fluorescence was also applied to determine herbicide 2,4dichlorophenoxyacetic acid (2,4-DCAA). A detection limit of $10^{-7} \mathrm{~mol} \mathrm{\textrm {dm } ^ { - 3 }}$ was determined. As stated above in the interaction with TNT, quenching was observed. The authors developed two inexpensive solid-state chips for the sensitive detection of 2,4DCAA and TNT by the assembly of the NBD- $\left(\mathrm{NH}_{2}\right)$-silica nanoparticles in the etched microwell array of a silicon wafer. 


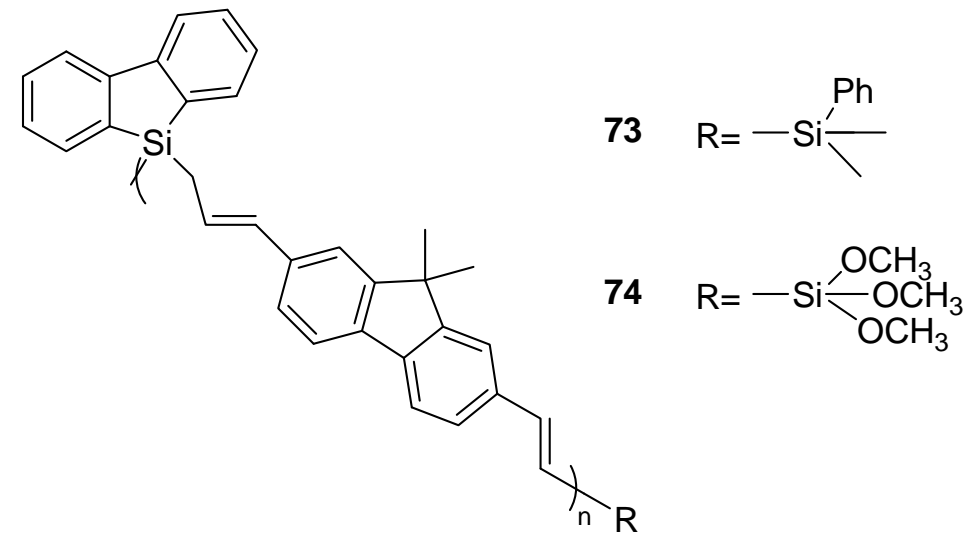

Fig. 32 Compounds 73 and 74

Trogler et al described the design of a blue emitting silafluorene-fluorene polymer (poly(silafluorene-(9,9-dimethyl-9H-fluorene)divinylene, PSFF) (73-74) (Fig. 32) on hollow silica nanoparticles as a sensing system for the detection of TNT and RDX in aqueous solutions. ${ }^{65}$ Fluorescence of the polymer in the solid state was attributed to aggregate-induced emission given the restricted intramolecular rotation. Three different methods were followed to include the PSFF polymer in silica nanoparticles: (a) polymer adsorption on the mesoporous surface (73); (b) covalent bonding of the polymer to the NPs surface (74); and (c) adsorption of the polymer onto octadecyl modified silica NPs (73). The highest quantum yield (0.13) was shown by the material to which PSFF was covalently attached. $\mathrm{K}_{\mathrm{SV}}$ (Stern-Volmer constant) for TNT detection with this system was 35 times higher with PSFF-coated silica NPs in water than with the toluene solution of the pure polymer. The authors reported detection limits of $200 \mathrm{ppb}$ for TNT and 800 ppb for RDX in aqueous solutions.
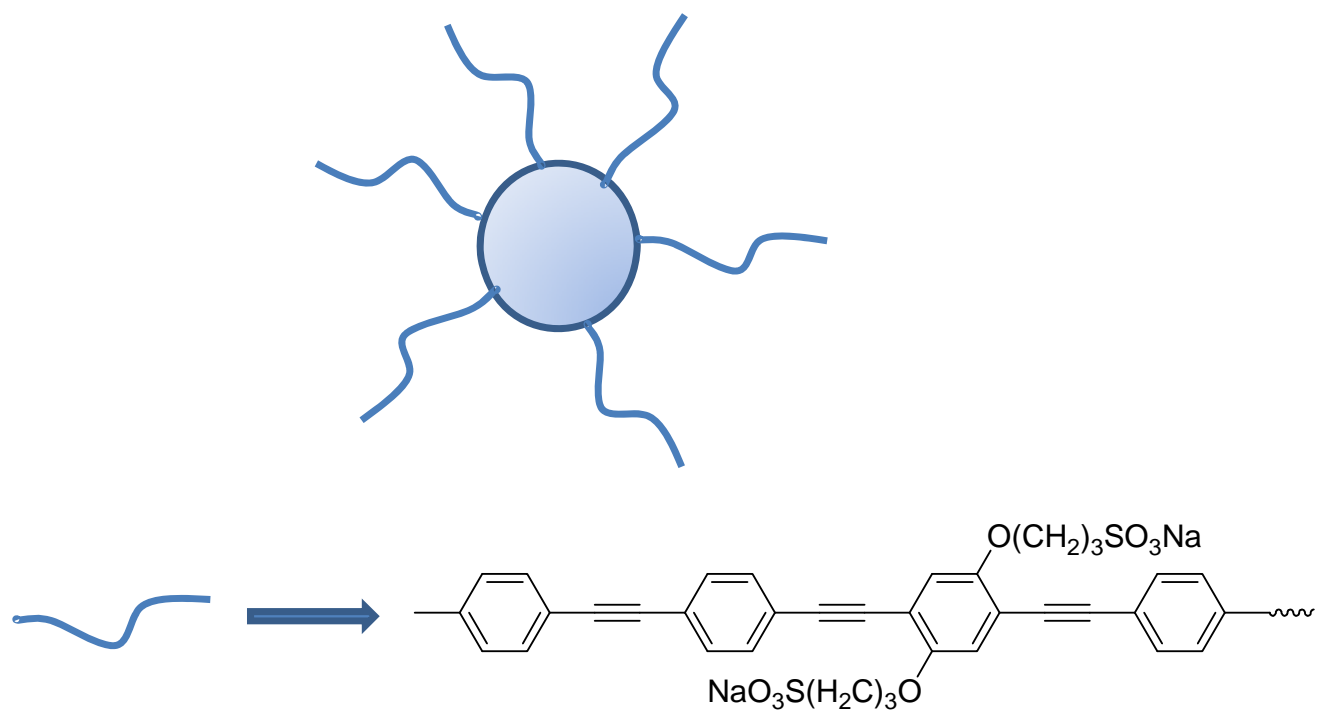

Fig. $33 \mathrm{SiO}_{2}$-PPS nanoparticles 
Recently, Yang and Li et al prepared different sized silica nanoparticles grafted with a derivative of polyphenyl acetylene. ${ }^{66}$ The response to TNT was examined in various solvents (methanol, DMSO): the $\mathrm{K}_{\mathrm{SV}}$ value increased when the size of the polymer-grafted silica nanoparticles decreased. Particles (Fig. 33) exhibited high sensitivity to TNT with a detection limit as low as $1 \mu \mathrm{M}$.

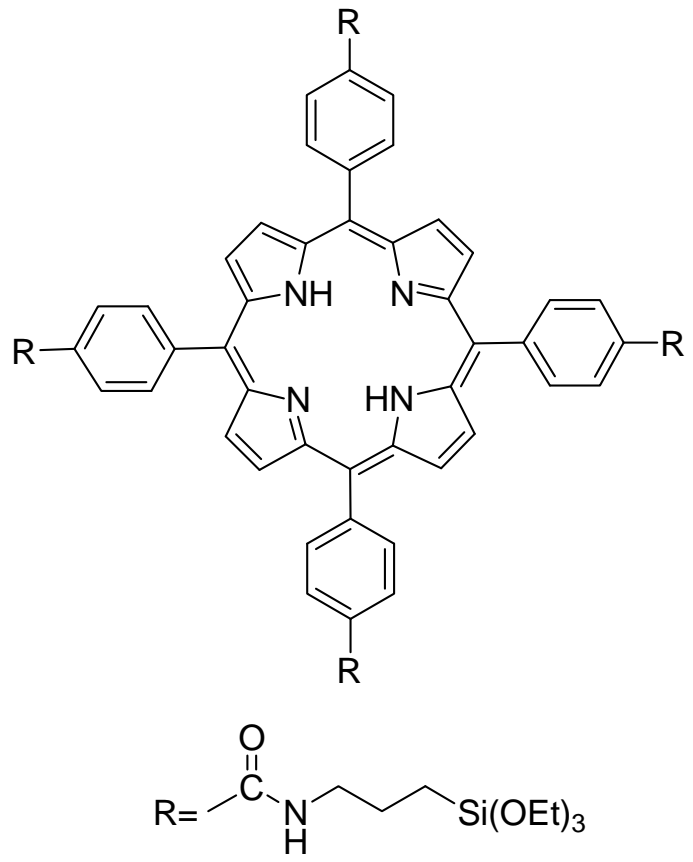

75<smiles></smiles>

76

Fig. 34 Compound 75 and 76

Dye-functionalised silica films used as materials for the detection of explosives have been reported by several authors. In an early work, Li and coworkers developed a hierarchically structured silica nanocomposite consisting of ordered macropores, surrounded by mesostructured walls of porphyrin (75)-functionalised silica as a highperformance chemosensory material (Fig. 34) showing considerably enhanced sensing behaviour for the detection of TNT vapours; i.e., sensitivity was nearly twice that observed in conjugated polymer-based TNT sensors. ${ }^{67}$ The interaction of TNT with the material was explained by the well-known tendency of the imino hydrogen of the porphyrin (75) to form hydrogen bonds with the nitro groups of the nitroaromatics and via $\pi$-stacking interactions of porphyrin with TNT. Using the nanocomposite, quenchings of $55 \%$ after a 10 -s exposure to TNT vapours and of $97 \%$ after a 2 -min 
exposure were observed. Apparently, the unique bimodal porous structure provided the necessary condition for the facile diffusion of analytes, while the large surface area substantially enhanced the interaction between TNT and the sensing elements. In a subsequent study, the same authors reported the successful synthesis of a series of porphyrin- and metalloporphyrin-doped silica films with a bimodal porous structure, which was constructed using polystyrene spheres and a surfactant (CTAB) as the structure-directing agent. ${ }^{68}$ This new kind of porphyrin-doped silica film displayed excellent fluorescence quenching in the presence of trace TNT vapours (55\% quenching after $10 \mathrm{~s}$ for $10 \mathrm{ppb}$ of TNT). As in the above case, the hierarchically porous structure provided a necessary condition for the facile diffusion of analytes, while the large surface area considerably enhanced the number of accessible sites.

Using a similar approach, the same authors described the preparation of porphyrin (75) and metalloporphyrin (cobalt and cadmium)-doped mesostructured silica films using small molecular or macromolecular surfactants as structure-directing agents. ${ }^{69}$ Two main key factors were investigated: the appropriate permeation of the analyte through the film and the analyte's affinity with the emissive centres. To study the first factor, a comparison of the time-dependent fluorescence quenching of the different silica matrixes upon exposure to nitroaromatic vapour was carried out. The worst response was obtained for amorphous silica films, while the response for mesoporous silica films was seen to be dependent on the surfactant used. In relation to the affinity between analytes and emissive components, the study demonstrated that the signal was better for those films containing metalloprophyrin units than for those containing freebase porphyrin. The cadmium-porphyrin-doped film presented the highest fluorescence quenching (near $56 \%$ after 10 s of exposure). In another work, the same authors obtained similar results when the dip-coating technique was used for film preparation instead of the spin-coating procedure, as described in the example above. Hence, the authors reported the synthesis of porphyrin (75)-doped silica films with a mesoporous structure which showed high fluorescence quenching sensitivity to the vapours (10 ppb) of TNT and other explosives. ${ }^{70}$ The mesostructure of the deposited silica film was strongly dependent on the reactants' mole ratio, and two different mesostructured films were prepared: one with one-dimensional pore channels oriented in parallel to the substrate; the other with a worm-like mesostructure. The latter film exhibited the highest response: $27 \%$ quenching after 10 s of exposure. 
In all the examples used to demonstrate performance as sensors of functionalised silica films, it is quite clear that the porous structure controlled the explosive molecules' accessibility to the signalling centres. Hence, special care has been paid to control film porosity, normally through the use of several different types of templates. However, it is also possible to prepare porous silica films using template-free sol-gel methods. Along this line, Bayindir et al prepared highly porous and transparent organic-modified silica aerogel films with stable fluorescence properties for the detection of gaseous TNT and 2,4-DNT. ${ }^{71}$ Maintenance of a porous network during solvent evaporation under ambient conditions was achieved by using the monomer methyltrimetoxysilane, which also provided the network with hydrophobic behaviour. A porphyrin derivative (76) was used as a fluorescent dopant with no modifications made for covalent linking.

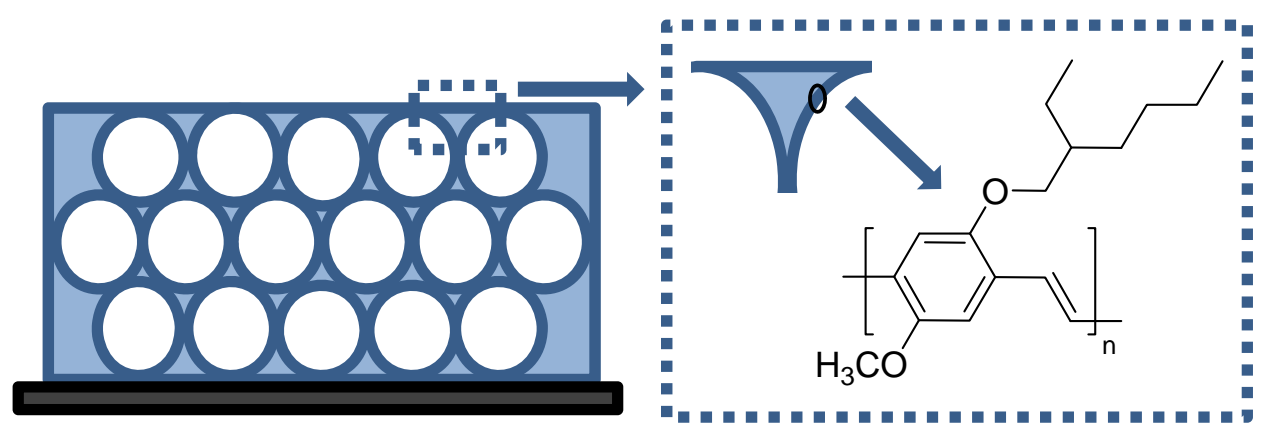

Fig. 35 Inverse opal photonic crystals coated with 21 on a porous surface for TNT detection

In another interesting work, Wang and co-workers reported the application of the light manipulation property of inverse opal photonic crystals for TNT detection. ${ }^{72}$ In order to obtain information about the porous structure's influence on fluorescence amplification, these authors prepared inverse opal photonic crystals in a range of macropore diameters that went from 225 to $520 \mathrm{~nm}$ (Fig. 35). The fluorescence intensities of all the photonic crystal-based sensors were obviously higher than those of the control sample (a porous non-periodic material). Those with the lesser increased fluorescence (18.6-fold enhancement) were photonic crystal whose stopband was far away from the absorption band of the deposited fluorophore (poly-[2-methoxy-5-(2ethylhexyloxy)-p-phenylenevinylene], 21). The films with the greatest enhancement (39.4-fold and 60.6-fold) were those whose stopband overlapped the emission spectrum of 21. The film's sensing behaviour with the highest fluorescent amplification was 
tested in the presence of TNT-saturated vapour, and a quenching efficiency of ca. $80 \%$ was found.

Li et al. also used 75 to prepare porphyrin-doped silica nanoafibres to detect TNT. Fibres were generated by electrospinning silica precursor sols. The authors demonstrated that doped nanofibres were efficient sensing materials for the detection of trace vapours $(10 \mathrm{ppb})$ of $\mathrm{TNT}^{73}$ Incorporation of a small portion of organic components into silica gel can considerably improve fibre formability, and allowed the preparation of a non-woven mesh of functionalised silica fibres, which can be handled like tissue paper. Membranes showed enough mechanical strength and flexibility, which are crucial for most applications. The nanofibres in fluorescence-based sensing materials with smaller diameters presented higher sensitivity, which was probably due to a larger amount of porphyrin molecules on the surface.

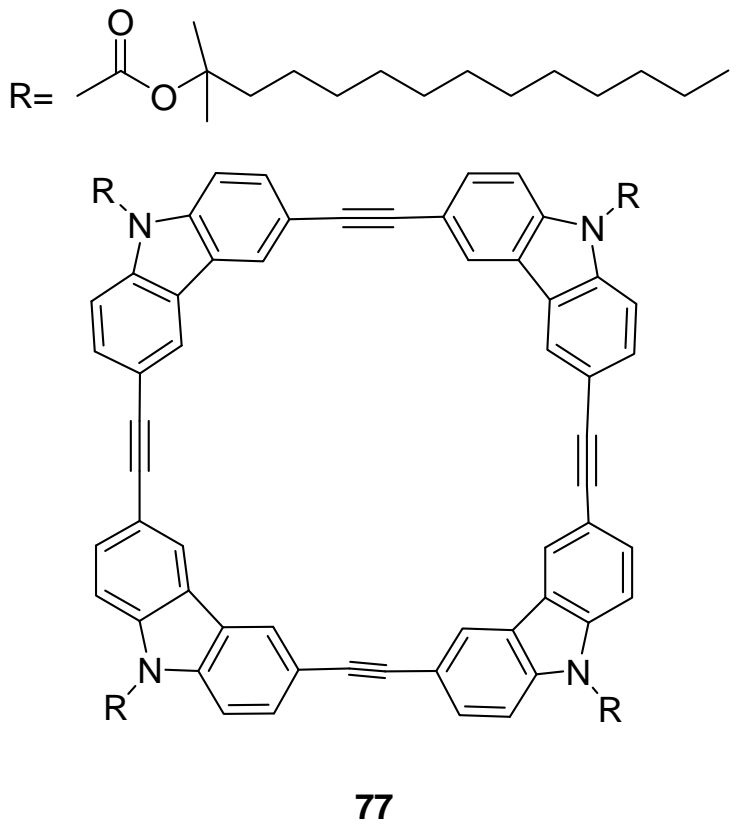

Fig. 36 Compound 77

In relation to the above functionalised silica films, a different type of fluorescence sensory material, with no silica support, was prepared using an arylene-ethynylene macrocycle (77) (Fig. 36). By means of the surface casting method, the authors prepared films of different thicknesses based on the micrometric nanofibres of the macrocycle. ${ }^{74}$ These films presented very effective fluorescence quenching upon exposure to the saturated vapour of 2,4-DNT and TNT. Quenching displayed little dependence on film thickness due to macrocycle's porous morphology and extended one-dimensional $\pi-\pi$ staking, which facilitated quencher molecules' access to excited states. Film 
fluorescence was slowly recovered by exposure to air or was quickly recovered by exposure to hydrazine vapour. The film showed great stability against photobleaching and a similar efficient quenching response was obtained with repeated use.

A different approach to the detection of nitroaromatics (TNT, 2,4-DNT, and NB) reported by Trogler, Sailor et al was based on the photoluminiscence decrease of porous silicon materials in the presence of these compounds. ${ }^{75}$ Porous silicon is a high surfacearea network of $\mathrm{Si}$ nanocrystallites, and is produced by an electrochemical etching of single crystal Si wafers. These nanocrystals exhibit quantum confinement effects and emit photoluminiscence. Photoluminiscence intensity depends on the presence of surface adsorbates and can also be quenched by charge acceptors and/or corrosive molecules. Nitroaromatic molecules have two of these features which can be applied to their detection: first, they are good electron acceptors ("direct detection") and, second, nitro-compounds can be catalytically oxidised to $\mathrm{CO}_{2}, \mathrm{H}_{2} \mathrm{O}$ and $\mathrm{NO}_{2}$ at temperatures lower than those needed to oxidise almost all other potential organic interferents. The authors found that using photoluminiscence measurements on porous silicon discrimination between the nitroaromatic derivatives and several common atmospheric interferents such as benzene, hexane, toluene, isooctane and isoprene could be achieved. The detection limits for 2,4-DNT and TNT using this system were $2 \mathrm{ppb}$ and $1 \mathrm{ppb}$, respectively.

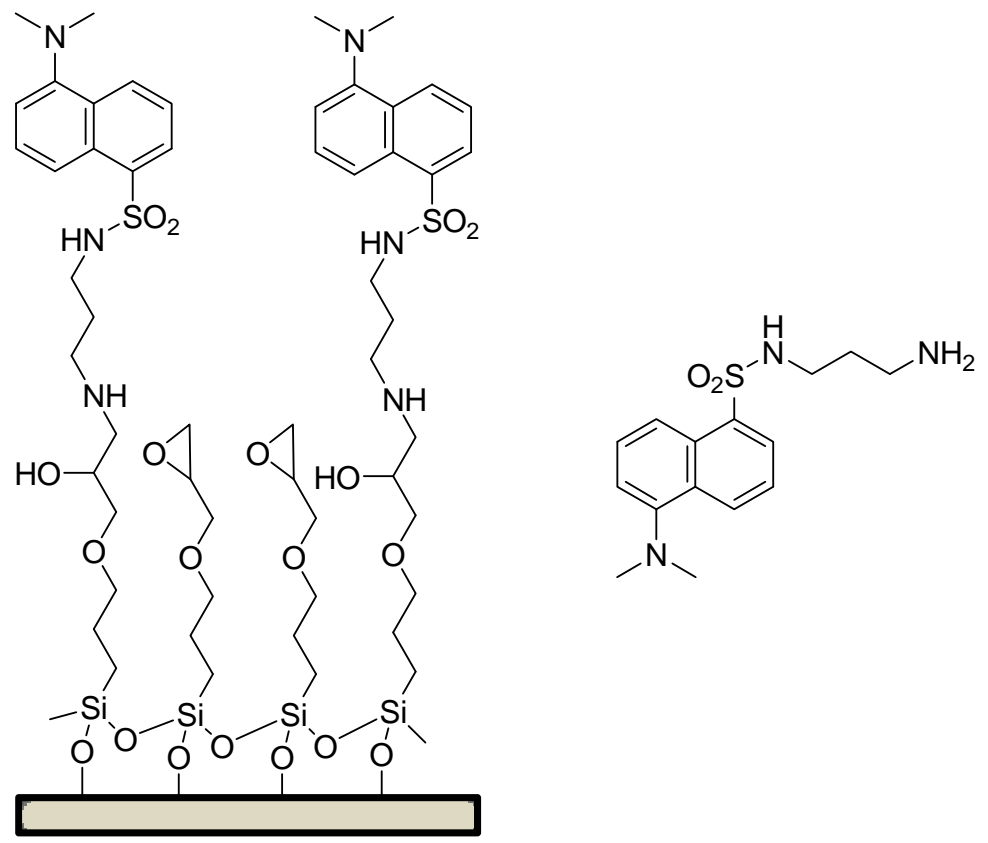

Fig. 37 Representation of the covalent immovilisation of dansyl on a glass slide surface 
Covalent attachment of different fluorophores to glass plate surfaces was a strategy applied by the group of Lü and Fang to accomplish sensing films for nitroaromatics (Fig. 37). One of the reported works was devoted to the study of a glass slide covalently functionalised with the small dansyl fluorophore via a relatively long, flexible spacer. ${ }^{76}$ By means of fluorescence quenching studies, the authors demonstrated how the system was not only selective to nitroaromatics in the aqueous phase, but was also quite sensitive as the detection limit of the film for TNT was as low as $10^{-6} \mathrm{~mol} \mathrm{~L}^{-1}$. The authors also found that nitroaromatics exhibited much greater quenching efficiencies in the emission of films than those observed for aromatics with no electronwithdrawing groups. They also observed that the higher the number of nitro-groups in the nitroaromatic derivative, the greater the quenching efficiency.

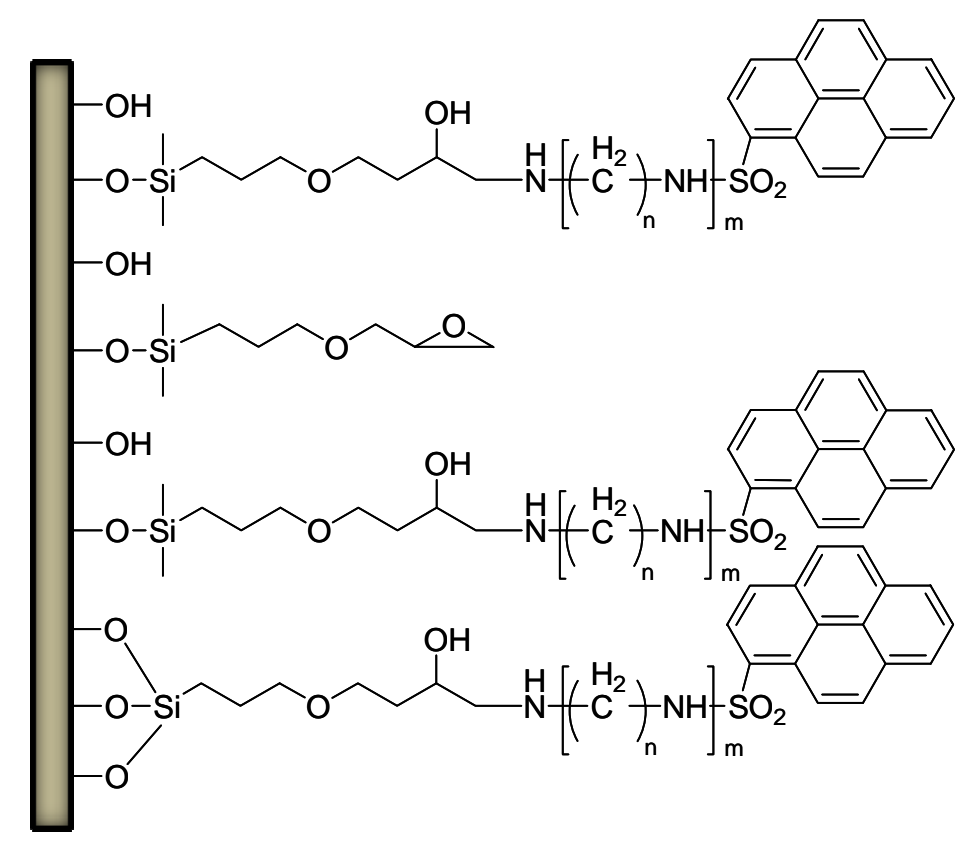

Fig. 38 Pyrene functionalised fluorescent film

The same group also reported a different functionalisation, employed to investigate the effect of some typical nitroaromatics in the vapour phase on a series of pyrene-anchored functionalised films with different densities and/or different spacer lengths (Fig. 38). ${ }^{77}$ The authors found that the greater the loading density, the higher the excimer/monomer intensity ratio of the pyrene. Fluorescence quenching was observed in the presence of NB, 2,4-DNT and TNT. The authors also observed that the response rate of films followed an order of NB $>2,4-\mathrm{DNT}>\mathrm{TNT}$, which is in accordance with the saturated vapour pressure order. In relation to the spacer effect, the intermediate 
length system (diamino propane subunit) proved the most sensitive to the presence of TNT and NB. On the other hand, better performance was seen with the lower loaded film as this consumes less quencher molecules to achieve similar quenching efficiency. The response of films was reversible and their performance was not affected by common interferents such as vapours of organic solvents, benzene, toluene, ethanol, perfume and smoke.

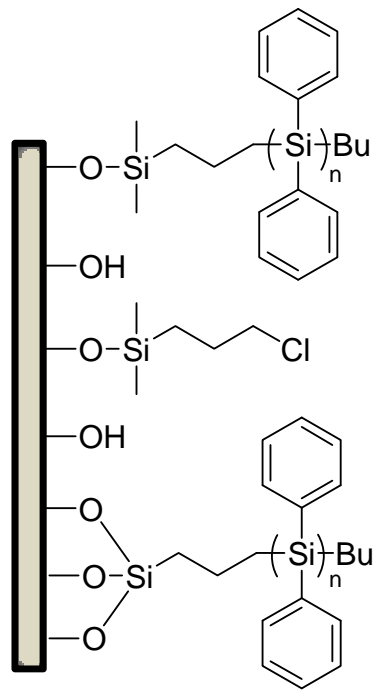

Fig. 39 Poly(diphenylsilane)s coupling onto a glass plate surface.

The same authors reported the detection of nitroaromatic compounds in the vapour phase by means of a fluorescence film sensor prepared by the chemical assembly of oligo(diphenylsilane)s on a glass plate surface (Fig. 39) ${ }^{78}$ For TNT, 55\% reduction of fluorescence emission after 10s of exposure was reported. By comparing the quenching results from static measurements with those of fluorescence lifetime measurements, the authors concluded that quenching was mainly caused by formation of a non-fluorescent donor-acceptor complex between conjugated polymers and nitroaromatic derivatives.

\section{3.- Single fluorophores}

\subsection{1.- Polycyclic aromatic hydrocarbons}

Polycyclic aromatic hydrocarbons are organic molecules that consist in fused aromatic rings without the presence of heteroatoms. Their fairly well-known photophysical properties, relatively high quantum yields and the fact that there is a large number of commercially available derivatives, make them a clear choice for the 
preparation of fluorescent receptors to detect neutral and charged species. One remarkable feature of polycyclic aromatic hydrocarbons is their electron donor nature due to their electron-rich structure, which allows the formation of charge transfer complexes with electron acceptor molecules. For this reason, polycyclic aromatic hydrocarbons (such as pyrene, perylene, anthracene, binaphtyl and fluorene) have been employed as receptors for nitrated explosives by means of a signalling event based on the latter's ability to quench the excited state of the former. This simple approach has been explored by McGuffin et al who reported the separation of complex explosive mixtures (also containing their degradation products) with capillary liquid chromatography and the individual detection of nitrated aromatic derivatives by measuring pyrene emission quenching by laser-induced fluorescence. ${ }^{79}$ The interaction mechanism relies on the formation of charge-transfer complexes between the electronrich pyrene and the electron withdrawing nitrated explosives. For nitroaromatic explosives, these authors found that the Stern-Volmer quenching constants increased with the number of nitro groups (TNT $>2,4-\mathrm{DNT}>2-\mathrm{NT}$ ), whereas the effectiveness of quenching did not follow this trend for non-aromatic nitrated explosives. The method showed certain interferences and other species such as organic amines, nitriles, halides and inorganic anions also induced a partial quenching of pyrene emission. This method was successfully applied to determine RDX, HMX, TNT, NM and ammonium nitrate in various commercial explosive samples.

Anslyn et al used simple fluorescent polycyclic aromatic hydrocarbons (pyrene, perylene and diphenylanthracene) located inside micelles to develop a differential array for the detection and identification of TNT, Tetryl, RDX and HMX explosives. ${ }^{80}$ These authors studied pyrene emission quenching efficiency by nitrated explosives in aqueous solutions containing Tween 80. Under these conditions, the monomer emission of pyrene was quenched to different extents upon the addition of TNT, Tetryl, RDX and HMX. However, the emission modulation observed was insufficient for accurate differentiation. It was observed that the excimer emission of the pyrene in aqueous solutions containing Tween 80 was also quenched upon the addition of TNT and RDX. TNT and RDX could be differentiated by using the ratio between monomer and excimer emission. The authors also studied the quenching of pyrene and perylene fluorophores co-dissolved in Tween 80 in the presence of nitrated explosives. In this system, excitation of pyrene resulted in some relaxation via the emission of the pyrene monomer along with some FRET to excite perylene, which then relaxes through its own emission. 
Addition of increasing quantities of TNT and RDX induced the gradual quenching of both emission bands. Finally, diphenylanthracene was also included in the Tween 80 micelles, and the changes in emission in the presence of nitrated derivatives were studied. In this case, addition of RDX and HMX induced negligible changes in the emission profile of the fluorophore, whereas the addition of TNT resulted in quenching due to a simple absorption of excitation light by TNT. The authors designed a sensory array consisting in several solutions of the above-cited fluorophores in Tween 80 . Examination of the array's fluorescence response in the presence of nitrated explosives using linear discriminant analysis (LDA) resulted in a "fingerprint" for each analyte.

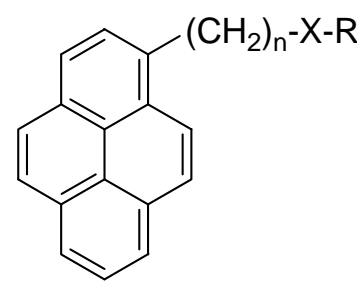

$78 \mathrm{n}=0, \mathrm{R}=\mathrm{Ph}$

$79 \mathrm{n}=1, \mathrm{R}=\mathrm{Ph}$

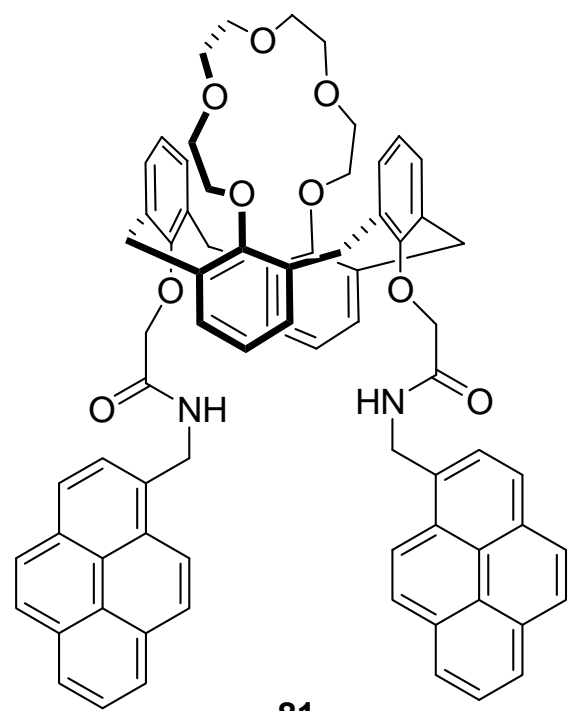

$80 \mathrm{n}=1, \mathrm{R}=\mathrm{p}-\mathrm{PhOMe}$

$$
\begin{aligned}
& \text { a } X=S \\
& \text { b } X=S(O) \\
& \text { c } X=S_{2}
\end{aligned}
$$

Fig. 40 Compounds 78-81

Pyrene derivatives containing sulfoxide moieties (78b, 79b and 80b) (Fig. 40) were used for the indirect fluorescence detection of TATP in dichloromethane. ${ }^{81}$ The authors demonstrated how the UV irradiation of TATP and MTO (methyltrioxorhenium) in dichloromethane solutions of $\mathbf{8 0 b}$ induced photolysis of TATP, mediated by MTO, to yield $\mathrm{H}_{2} \mathrm{O}_{2}$, which induced the oxidation of sulfoxide $\mathbf{8 0 b}$ to yield the highly emissive sulfone 80c. A maximum 50-fold increase in the emission intensity at $400 \mathrm{~nm}$ after a 90 -minute reaction was observed. Almost the same response was obtained with 78b and 79b. By using this indirect method, TATP concentrations as 
low as $100 \mathrm{nmol}$ were detected. The selectivity of the method proved remarkable because other oxidants such as $t-\mathrm{BuOOH}, \mathrm{NaOCl}, \mathrm{LiClO}_{4}, \mathrm{~K}_{2} \mathrm{Cr}_{2} \mathrm{O}_{7}$, or air, were unable to oxidise sulfoxides to the corresponding emissive sulfones, whereas $\mathrm{KMnO}_{4}$ was a clear interferent (it was able to induce the sulfoxide-sulfone reaction).

Scaiano et al. also employed pyrene as an electron-rich molecule to detect nitro explosives by emission quenching. ${ }^{82}$ In this case, ethanolic solutions of pyrene excited at $355 \mathrm{~nm}$ showed two distinct emission bands: one finely structured band below 400 $\mathrm{nm}$ corresponding to the emission from the singlet excited state monomer; one broad band at $470 \mathrm{~nm}$ due to the formation of excimers. Both emission bands were quenched upon the addition of electron-deficient nitro derivatives such as NB, 1,4-DNB, 5-NX, 1,3-DNB, NM and NE, whereas electron-rich derivatives such as $N, N$-dimethylaniline, trioctylamine and $N, N$-dimethyl-2,6-diisopropylaniline did not significantly quench the pyrene emission. From an analytical point of view, the ratio between monomer intensity and excimer emission $\left(\mathrm{F}_{\mathrm{M}} / \mathrm{F}_{\mathrm{E}}\right)$ as a function of the quencher concentration allowed the discrimination of different nitro derivatives.

The dipyrenylamidocalix[4]arene-[15]crown-5 (81) receptor was used for the colorimetric and fluorimetric detection of nitrated explosives. ${ }^{83}$ Acetonitrile solutions of receptor 81 presented two emission bands at 375 (monomer) and $450 \mathrm{~nm}$ (excimer), when excited at $343 \mathrm{~nm}$. Addition of TNB and TNT to acetonitrile solutions of $\mathbf{8 1}$ induced a significant quenching of both emission bands, whereas addition of 2,6-DNT, 1,3-DNB and NB resulted in only moderate quenching. With receptor $\mathbf{8 1}$, TNT could be detected at concentrations as low as 1 ppb. Receptor $\mathbf{8 1}$ could also be used for the colorimetric detection of TNT and TNB. Thus, chloroform solutions of receptor $\mathbf{8 1}$ showed the typical intense absorption bands of pyrene in the $320-360 \mathrm{~nm}$ interval. With the addition of TNT, the initial colourless solution turned yellow, whereas it turned reddish-orange when TNB was added.

Seitz et al. prepared cellulose triacetate membranes which were plasticised with isodecyl diphenylphosphate, containing pyrenebutyric acid as a fluorophore for the detection of TNT, 2,4-DNT and RDX in water. ${ }^{84}$ The membrane was able to extract nitrated derivatives from water to induce the quenching of the emission band of pyreneburyric acid centred at $412 \mathrm{~nm}$ (excitation at $354 \mathrm{~nm}$ ). The detection limits obtained with this membrane were 2 ppm of TNT and 2,4-DNT and 10 ppm of RDX. Membrane sensitivity (TNT $>2$,4-DNT $>$ RDX) correlated well with the octanol-water partition coefficients of nitrated derivatives. 


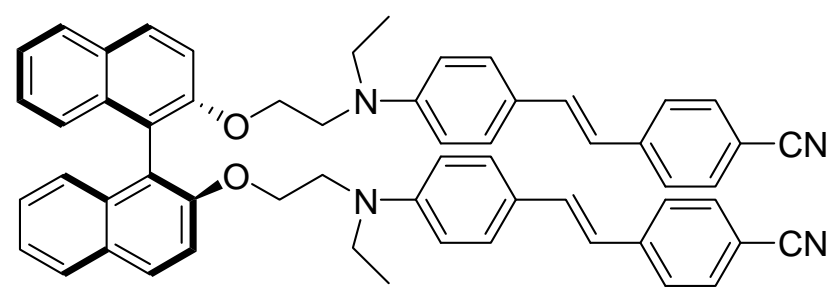

82

Fig. 41 Compound 82

Binaphtyl derivative 82 (Fig. 41), functionalised with donor-acceptor substituted trans-stilbenes, was used for the detection of 2,4-DNT and TNT. ${ }^{85}$ The films of 82, drop-casted from 1:3 v/v chloroform-toluene mixtures, showed an intense emission band at $510 \mathrm{~nm}$ which was quenched in the presence of 2,4-DNT (91\% quenching after a 10-minute exposure to saturated vapours) and TNT (72\% quenching after a 10-minute exposure to saturated vapours). The different quenching degree clearly related with the higher vapour pressure of 2,4-DNT when compared to that of TNT. The observed quenching was ascribed to a photo-induced electron transfer process from 82 to nitroaromatic quenchers.

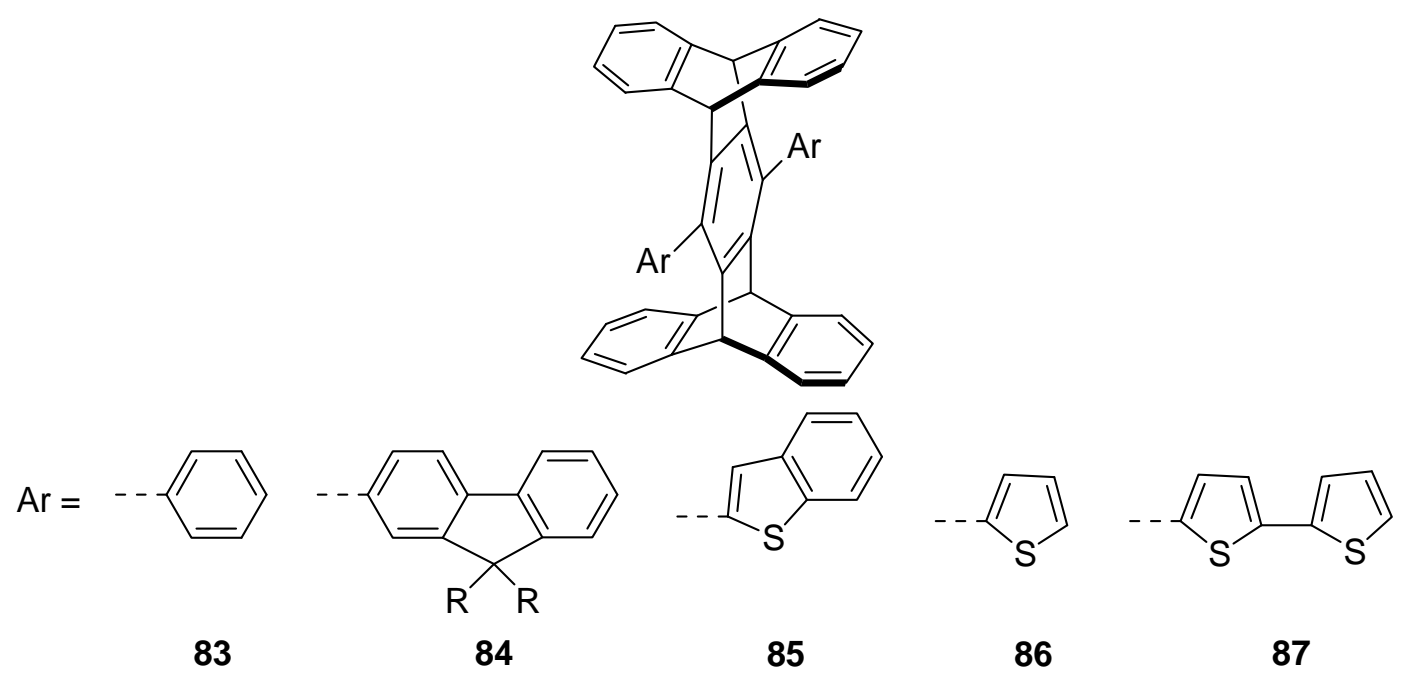

Fig. 42 Compounds 83-87

Anzenbacher Jr. et al reported a series of 1,4-diarylpentiptycenes 83-87 (Fig. 42) for the fluorescence detection of nitroaromatic derivatives. ${ }^{86}$ The dichloromethane solutions of receptor 87 showed an emission band at $450 \mathrm{~nm}$, which was gradually quenched upon the addition of increasing quantities of NB, 2,4-DNT, TNT, NN, 
DMNB. Virtually the same results were obtained with the dichloromethane solutions of 83-86 receptors. The authors also embedded receptors $\mathbf{8 6}$ and $\mathbf{8 7}$ in polyurethane films and demonstrated that emission was quenched upon exposure to 2,4-DNT and TNT vapours. Moreover, the fluorescence of the films was restored upon washing with a stream of clean air.

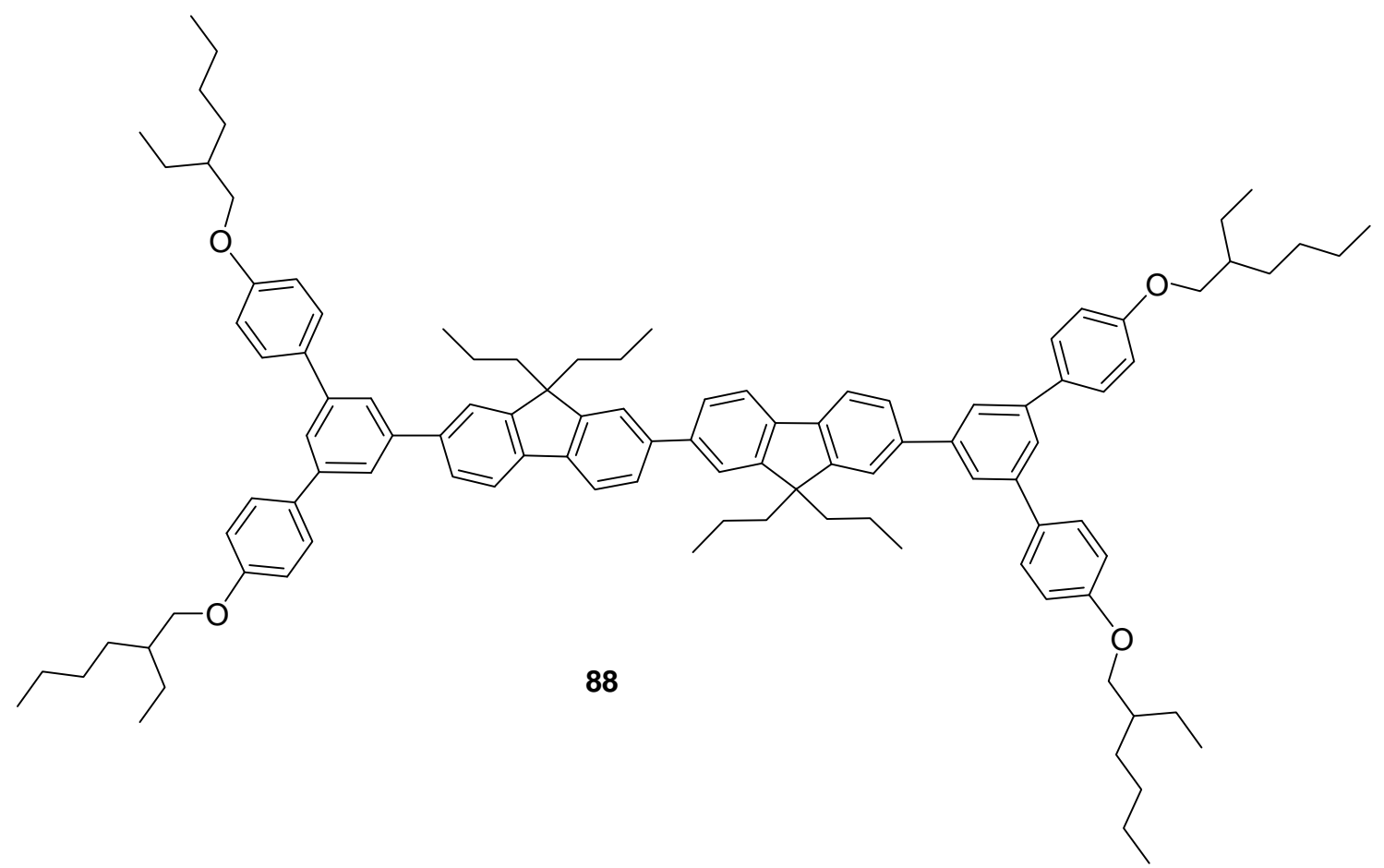

Fig. 43 Compound 88 


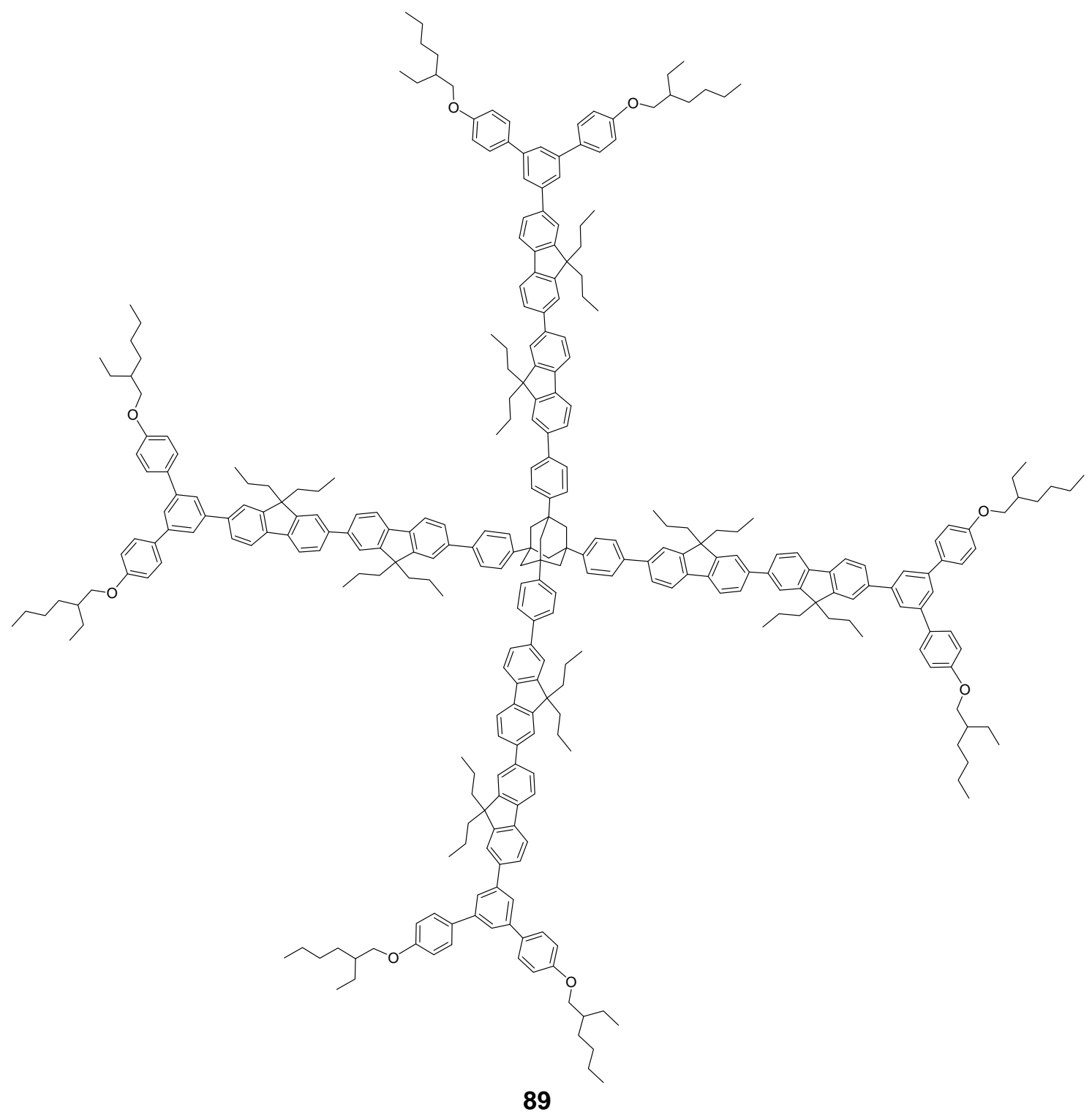

Fig. 44 Compound 89

Fluorescent dendrimers have been suggested as an alternative to organic polymers in the development of sensory materials for nitrated explosives. Although less accessible, they offer the advantage of being monodisperse and their structure can be modulated to control their response. Recently, a study into the photophysical properties of the bifluorene-containing dendrimers 88 (Fig. 43) and 89 (Fig. 44) as fluorescent materials to detect explosives, has been published. ${ }^{87}$ The spectroscopic behaviour of both dendrimers 88-89 was identical and the THF solutions of both derivatives showed an intense emission band at $393 \mathrm{~nm}$ with quantum yields of $90 \%$. The emission of the THF solutions of $\mathbf{8 8}$ and $\mathbf{8 9}$ was quenched upon the addition of 1,4-DNB, 2,4-DNT and 4-NT. Moreover, quenching efficiency was greater for the 3-dimensional dendrimer 89 than it was for $\mathbf{8 8}$. This enhanced quenching efficiency was ascribed to an amplification 
effect in 89, which has multiple identical chromophores that can all be quenched by a single analyte molecule. Emission quenching was ascribed to an efficient photo-induced electron transfer from excited bifluorene moieties to nitroaromatic explosives.

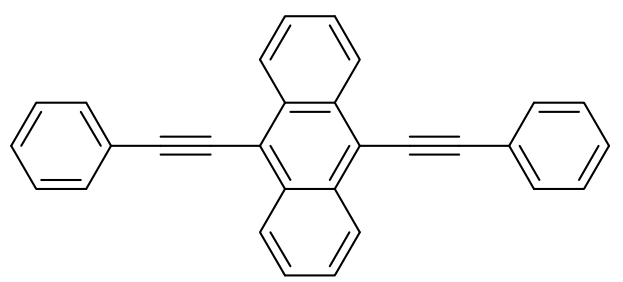

90

Fig. 45 Compound 90

The formation of inclusion complexes by $\gamma$-CD was used by the same authors for the fluorescence recognition of nitroaromatic explosives. ${ }^{88}$ Water-THF 95:5 v/v solutions of 9,10-bis(phenylethynyl)anthracene (90) (Fig. 45) presented an intense emission band upon excitation at $495 \mathrm{~nm}$, which was moderately quenched upon the addition of increasing quantities of $\gamma-\mathrm{CD}$. This emission quenching was ascribed to the formation of an inclusion complex between 90 and $\gamma$-CD. Upon the addition of nitroaromatic explosives, TNT and Tetryl, to solutions of the $\mathbf{9 0} \cdot(\gamma-\mathrm{CD})$ complex, the quenching of the fluorophore emission intensity was seen to become further intense. This quenching was ascribed to the formation of a charge transfer complex between the nitroaromatic explosive and $\mathbf{9 0}$ in the cavity of the $\gamma-\mathrm{CD}$. The same complex was formed in the absence of $\gamma-\mathrm{CD}$, but the observed emission quenching was poorer. Addition of RDX, HMX and PETN to the solutions of $\mathbf{9 0} \cdot(\gamma-\mathrm{CD})$ complex induced negligible changes in emission intensity.

\subsection{2.- Heterocycles}

Heterocycles containing more than one fused cycle (carbazoles, siloles, thiophenes, benzothiophenes and porphyrins) have been used as fluorophores in the design of receptors for the detection of explosive molecules. 

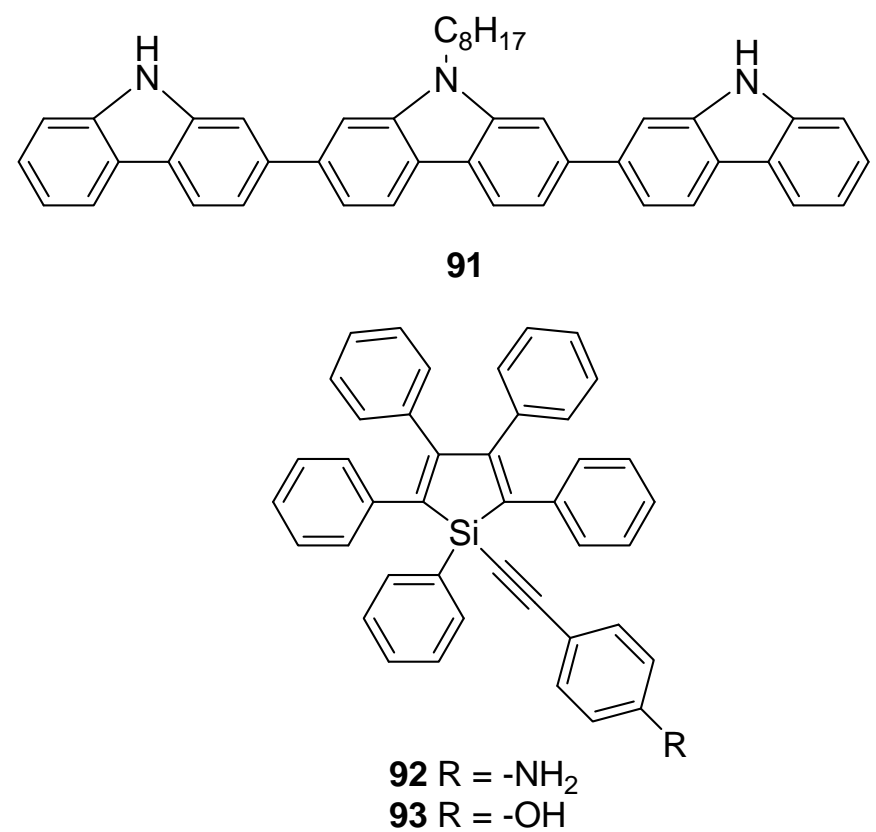

Fig. 46 Compounds 91-93

The linear 2,7-linked carbazole trimer (91) (Fig. 46) was used to prepare fluorescent nanofibrils (ca. $30 \mathrm{~nm}$ and lengths of several microns) which formed nanoporous films consisting of entangled fibril networks. ${ }^{89}$ The nanofibrils of $\mathbf{9 1}$ exhibited strong blue fluorescence at $438 \mathrm{~nm}$ upon excitation with UV light. These systems possess long-range exciton diffusion due to the extended intermolecular $\pi-\pi$ electronic interaction, tunable porosity and the large surface area:volume ratio, which is intrinsic to the nanofibril film. Films displayed potential applications in sensor devices for on-site explosives monitoring. Thus, efficient fluorescent quenching in the presence of TNT (70\% quenching after 60 seconds) and 2,4-DNT (50\% quenching after 10 seconds) vapours was observed. The same sensing films containing 91 were also used for the fluorescent detection of nitromethane. In all cases, the sensing mechanism relies on the photo-induced electron transfer between the carbazole trimer and explosives. Finally, fluorescence of nanofibrils can be recovered by heating films at $50^{\circ} \mathrm{C}$ in vacuum for 10 minutes.

As described above, siloles are a class of organometallic molecules which possess a unique silacyclopentadiene core structure and exhibit a number of interesting properties. One characteristic of silole derivatives is their aggregation-induced emission phenomenon; i.e., non-emissive molecules are induced to emit efficiently by aggregate formation, a totally opposite effect to that typically observed in common luminophores. 
Silole derivatives 92 and 93 were prepared by employing the terminal alkyne 1ethynylpentaphenylsilole via a palladium catalysed cross-coupling reaction with aryl halide derivatives. ${ }^{90}$ The THF-water 1:9 v/v solutions of 92 showed an intense emission band at $500 \mathrm{~nm}$ upon excitation at $370 \mathrm{~nm}$ due to the formation of highly luminescent aggregates. This emission band was quenched upon the addition of increasing quantities of PA. Nearly the same emission quenching with PA was obtained with receptor 93. The response of both receptors was quite selective to PA and addition of 2,4-DNT to THF-water 1:9 v/v solutions only induced moderate quenching. Moreover, the detection limits obtained for both receptors were ca. $0.1 \mathrm{ppm}$ of PA. The observed emission quenching was ascribed to the rupture of the aggregates of 92 and 93 upon coordination with PA.

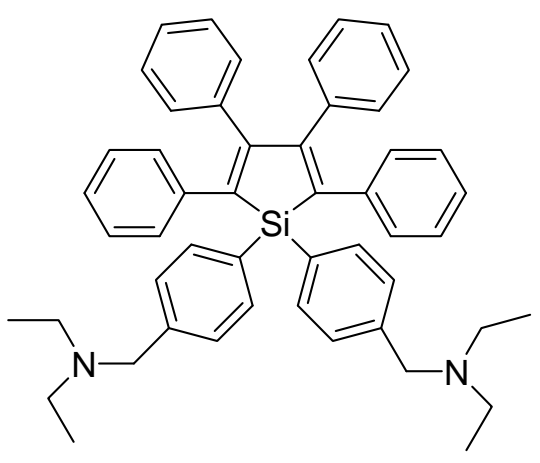

94

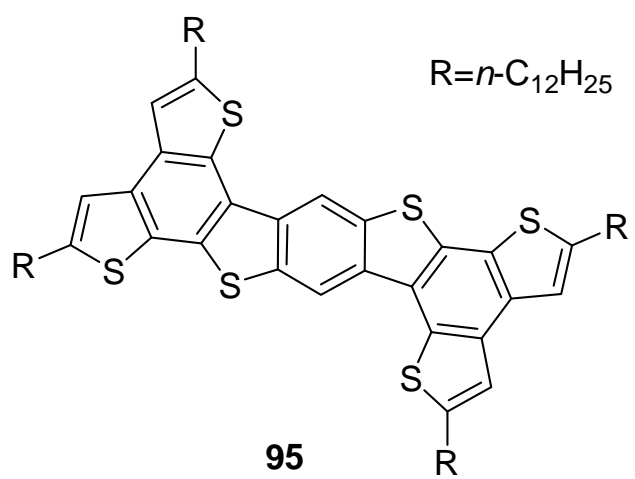

95

Fig. 47 Compounds 94 and 95

The aggregation-induced emission phenomenon was also observed for silole derivative 94 (Fig. 47). ${ }^{91}$ The THF-water 1:100 v/v solutions of receptor 94 showed an intense emission band centred at ca. $500 \mathrm{~nm}$ due to the formation of highly dispersed nano-sized aggregates. Addition of increasing quantities of PA to the solutions of receptor 94 induced a progressive quenching of the emission band and complete quenching was observed upon the addition of $5 \mu \mathrm{g} / \mathrm{mL}$ of PA. As in the previous example, the observed emission quenching was ascribed to the rupture of the aggregates of 94 upon coordination with PA.

Planar benzothiophene compound 95 was used for the preparation of 1D microbelts and 3D flower-shaped supernanostructures through $\pi$ - $\pi$ stacking and van der Waals interactions. ${ }^{92} 1 \mathrm{D}$ microbelts were self-assembled from 95 in 1,4-dioxane after the solution was drop-casted onto a glass substrate and the solvent was evaporated. By 
replacing the 1,4-dioxane with THF and $n$-decane, and after drop-casting onto glass substrates, two 3D flower-shaped supernanostructures (A and B, respectively) were obtained. Upon excitation of the films of these nanostructures, intense emission bands in the 450-480 nm interval were observed. Exposure of the films of flower A and flower B to 2,4-DNT vapours (ca. $100 \mathrm{ppb}$ ) induced a quenching of emission intensity to half the original intensity after 330 and 30 seconds for flower A and flower B, respectively. However for the 1D microbelts, a half emission quenching was achieved after 22000 seconds. These high response speeds observed for flowers A and B, when compared with those obtained with 1D microbelts, were ascribed to the large surface area and the more porous morphology of the former. Practically the same quenching effects, but at lower speeds, were obtained for the three nanomaterials in the presence of TNT vapour (ca. $5 \mathrm{ppb}$ ). The detection mechanism was attributed to an electron transfer process from the electron-rich organic materials to electron-deficient nitroaromatic compounds. This is a good example of how morphology control through self-assembly provides an interesting platform to develop organic crystalline microstructures to design chemosensors in which differences in film morphologies lead to dramatic changes in explosive detection speed.

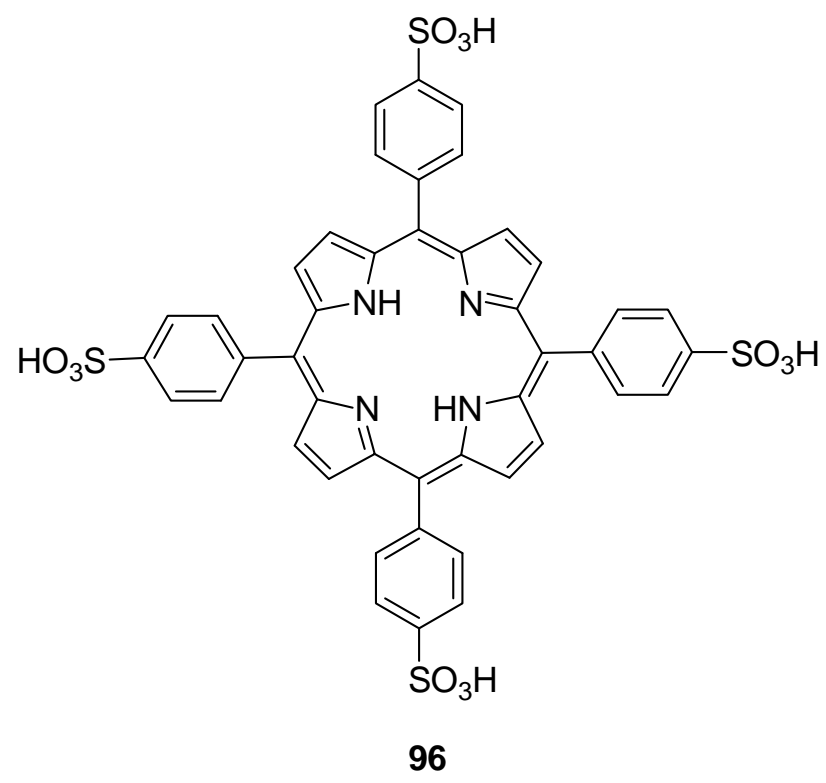

Fig. 48 Compound 96

Meso-tetra(4-sulfonatophenyl)porphyrin (96) (Fig. 48) was prepared and used for the fluorescence detection of TNT in water. ${ }^{93}$ Aqueous solutions of 96 buffered at pH 7.0 showed two emission bands at 645 and $702 \mathrm{~nm}$ upon excitation at $413 \mathrm{~nm}$. Addition 
of increasing quantities of TNT resulted in the formation of a non-emissive 96-TNT complex. By taking emission quenching measurements, the authors reported a detection limit of $200 \mathrm{ppb}$ for TNT.

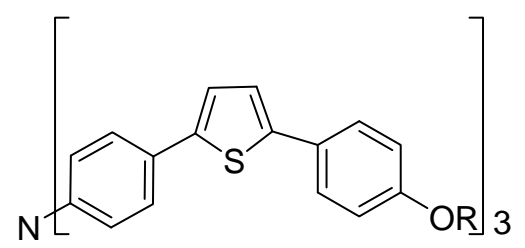

97

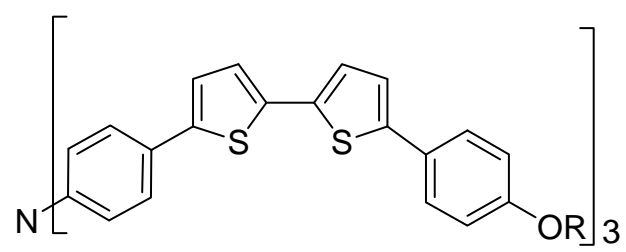

98<smiles>CCCCC(CC)CC</smiles><smiles>[R]Oc1ccc(-c2cc(-c3ccc(O)cc3)cc(-c3ccc(-c4ccc(O)cc4)s3)c2)cc1</smiles>

99<smiles>[R]Oc1ccc(-c2cc(-c3ccc([R])cc3)cc(-c3ccc(-c4ccc(-c5ccc(N)cc5)s4)s3)c2)cc1</smiles>

100

Fig. 49 Compounds 97-100

Branched fluorescent receptors containing thiophene units in the arms and triphenylamine centres, 97-100 (Fig. 49), were prepared and used for the detection of nitrated compounds such as 2,4-DNT, 1,4-DNB, plastic explosive DMNB and 4-NT. ${ }^{94}$ The THF solutions of 97-100 exhibited intense emission bands in the 450-650 nm range upon excitation at $400 \mathrm{~nm}$. Addition of increasing quantities of 2,4-DNT, 1,4-DNB, 4NT and DMNB induced different degrees of quenching (more intensity for the thiophene-containing $\mathbf{9 7}$ and 99 dendrimers) due to an efficient photo-induced electron transfer involving electron-rich dendrimers and nitro derivatives. The greatest quenching was observed for 1,4-DNB followed by 2,4-DNT, 4-NT and DMNB, the observed trend clearly relating with the reduction potential of nitrated compounds. Time-resolved measurements demonstrated that, in contrast to conjugated polymers, quenching is dominated by collisional interactions between the dendrimers and the nitro derivatives. 


\subsection{3.- Metal complexes}

Fluorophores containing metal complexes have also been used for the detection of nitrated explosives by emission quenching. One of the first examples used poly(amidoamine) dendrimers (PAMAM) functionalised with pendant $\left[\mathrm{Ru}(\mathrm{bpy})_{3}\right]^{2+}$ fluorophores of G0-to-G4 generations, which were employed for the detection of TNT and 2,4-DNT. ${ }^{95}$ Ethanol-water 3:7 v/v solutions of dendrimers showed the typical emission band of the $\left[\mathrm{Ru}(\mathrm{bpy})_{3}\right]^{2+}$ complex in the $575-640 \mathrm{~nm}$ range. Addition of increasing quantities of TNT and DNT induced the quenching of the emission intensity of dendrimers, whereas addition of NT induced negligible changes. The most intense quenching was observed for TNT. Additionally, the greatest quenching efficiency was noted for dendrimer G2, which was the dendrimer with a more "open" structure, thereby exhibiting fine accessibility to the pendant $\left[\mathrm{Ru}(\mathrm{bpy})_{3}\right]^{2+}$ fluorophores.

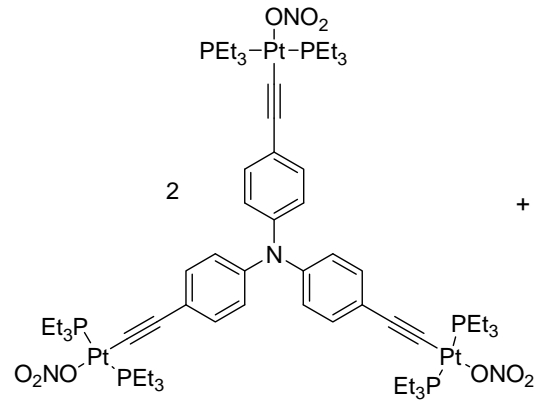

M

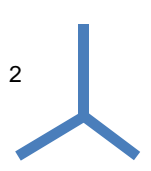

(c)

$\mathrm{M}_{\mathrm{b}}$

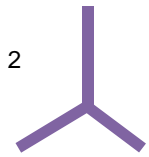

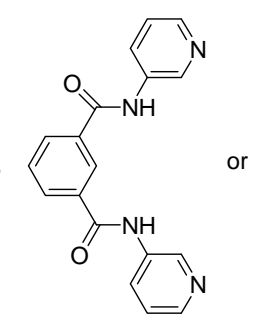

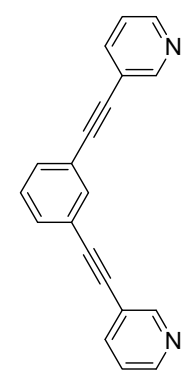

$\mathrm{L}_{\mathrm{a}}$

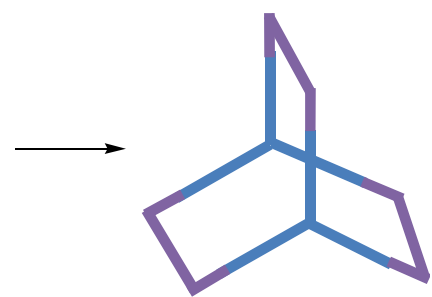

$\mathrm{L}$

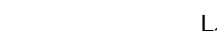

3
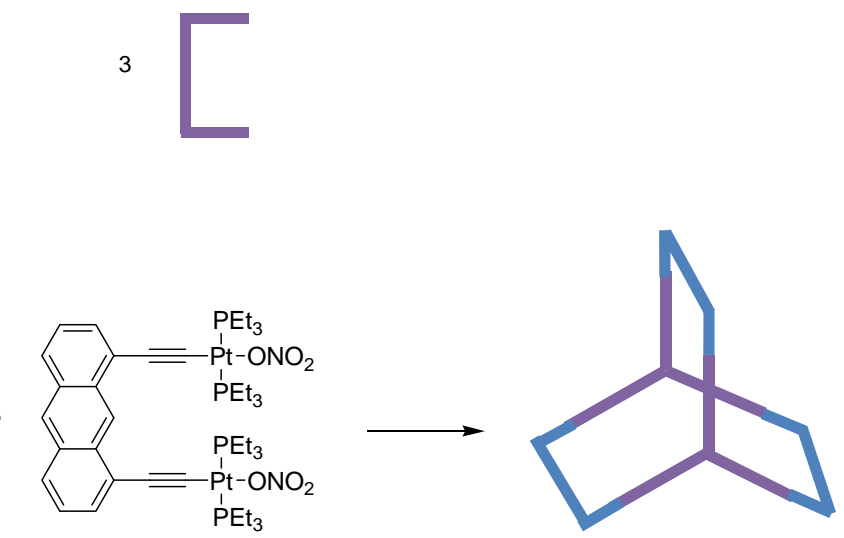

3

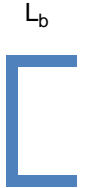

$101 b$ 


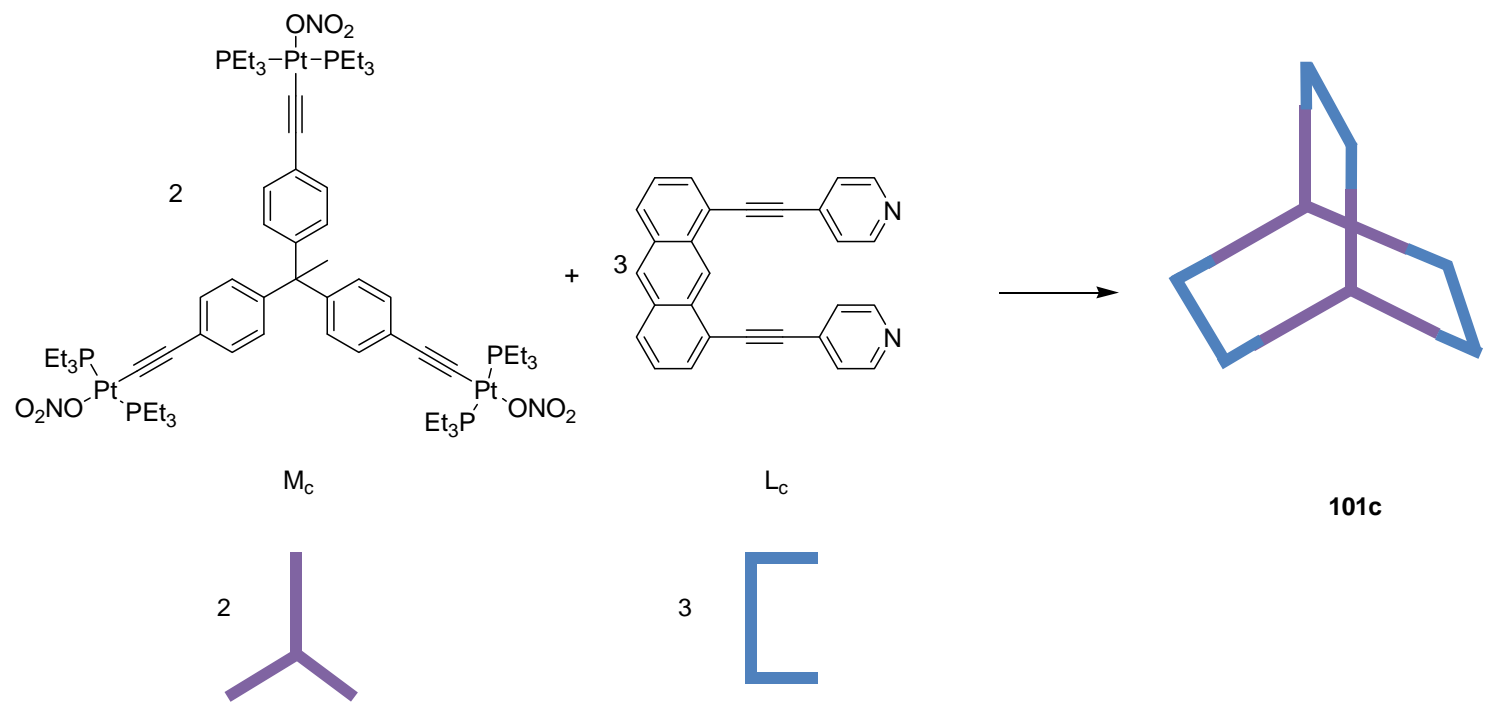

Fig. 50 Schematic representation of the formation of 101, 101a, 101b and

101c from $M$ and $L$ respectively

Mukherjee et al reported some $\mathrm{Pd}$ and $\mathrm{Pt}$ complexes for the detection of nitroaromatic explosives. In a first report, they designed and synthesised a nanoscopic trigonal prismatic cage $\mathrm{M}_{2} \mathrm{~L}_{3}$ (101) obtained by the self-assembly of the organometallic $\mathrm{Pt}_{3}$ tritopic planar acceptor 4,4',4',-tris[ethynyl-trans-Pt $\left(\mathrm{PEt}_{3}\right)_{2}\left(\mathrm{NO}_{3}\right)$ ] triphenylamine (M) with a clip-type amide containing the ligand 1,3-bis(3-pyridyl)isophthalic amide (L) (Fig. 50). ${ }^{96}$ Ethynyl functionality was introduced to make the linker rigid and planar, and to make it particularly fluorescent since it has been found that coordination complexes containing this functional group frequently show fluorescent/luminescent behaviour. Complex 101 in $\mathrm{CH}_{2} \mathrm{Cl}_{2}$-DMF 4:1 v/v solution showed two absorption bands at $275 \mathrm{~nm}$ (related with a $\pi-\pi^{*}$ transition) and $368 \mathrm{~nm}$ (attributed to a metal-to-ligand charge transfer). Upon excitation at $400 \mathrm{~nm}$, a broad emission band at $546 \mathrm{~nm}$ was observed. This emission was gradually quenched upon the addition of increasing quantities of TNT. The quenching of the emission intensity of $\mathbf{1 0 1}$ was ascribed to a photo-induced electron transfer from the excited state of the complex to the nitroaromatic compound.

In another work, the same authors reported the synthesis of a new trigonal prism 101a from $\mathbf{M}$ using the ethynyl-containing donor clip 1,3-bis(3-pyridylethynyl)benzene) (La). A complementary approach was also followed to prepare not only $\mathbf{1 0 1 b}$ from the 
organic tritopic planar donor 4,4',4',-tris(4-pyridylethynyl)triphenylamine (Mb) in combination with an organometallic $\mathrm{Pt}_{2}$-clip containing an anthracene with ethynyl functionality (Lb), but also 101c from 4,4',4',-tris[ethynyl-trans$\left.\mathrm{Pt}\left(\mathrm{PEt}_{3}\right)_{2}\left(\mathrm{NO}_{3}\right)\right]$ triphenylethane (Mc) upon the reaction with [1,8-bis(4pyridylethynyl)anthracene] (Lc). ${ }^{97}$ The conjugated ethynyl bridges in 101a, 101b and 101c make these assemblies $\pi$-electron-rich and possible hosts for electron-deficient nitroaromatic compounds. DMF solutions of complexes 101a, 101b and 101c showed broad intense emission bands in the 420-700 $\mathrm{nm}$ range when excited at $400 \mathrm{~nm}$. The fluorescence of the DMF solutions of 101b and 101c was quenched upon the gradual addition of TNT. The quenching mechanism was ascribed to the formation of a charge transfer complex between the $\pi$-electron-rich cages and the electron-deficient TNT. The formation of these complexes favours a photo-induced electron transfer process from the excited states of cages to the ground state of TNT. The authors also studied the performance of spin-cast films prepared by the spin-coating of DMF solutions of 101c over quartz, and they observed a fluorescent quenching on exposure to TNT vapours.

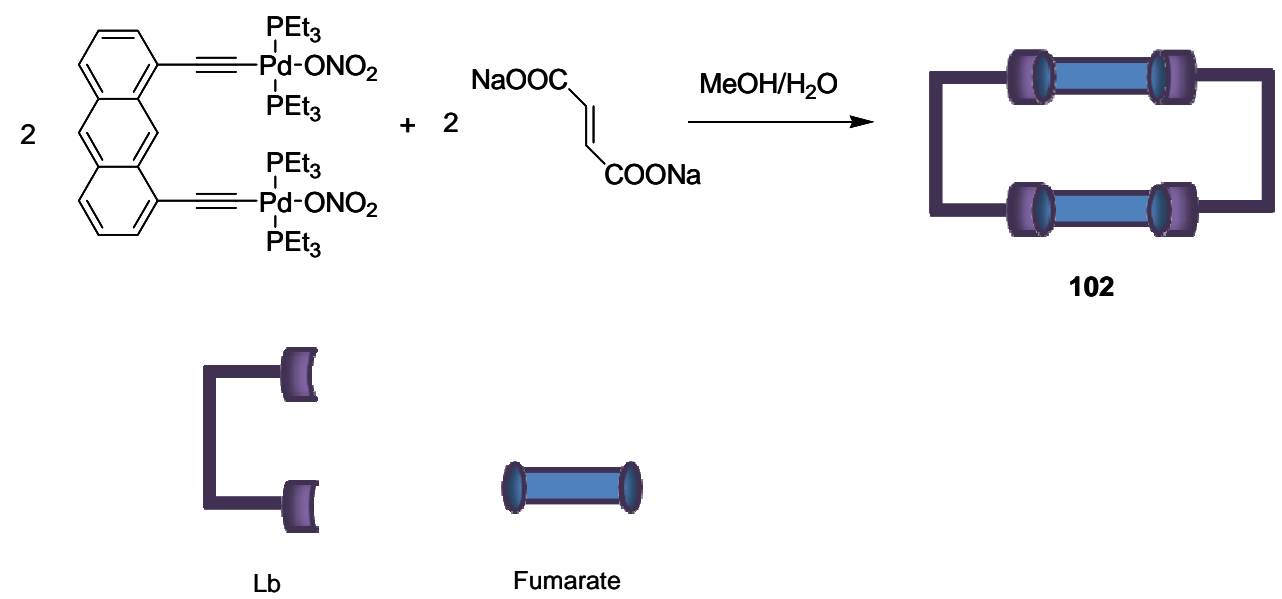

Fig. 51 Schematic representation of the formation of $\mathbf{1 0 2}$ from Mc and Fumarate

The same group also reported the synthesis of the molecular rectangle $\mathbf{1 0 2}$ through the [2+2] self-assembly of a $\mathrm{Pd}_{2}^{\mathrm{II}}$ shape selective organometallic molecular "clip" (Lb) with the linear dicarboxylate donor fumarate (Fig. 51). ${ }^{98}$ Chloroform solutions of rectangle 102 showed an intense emission band at $440 \mathrm{~nm}$, which was efficiently quenched upon the addition of increasing quantities of TNT with a detection limit of $1.0 \times 10^{-5} \mathrm{~mol} \mathrm{dm}^{-3}$. 


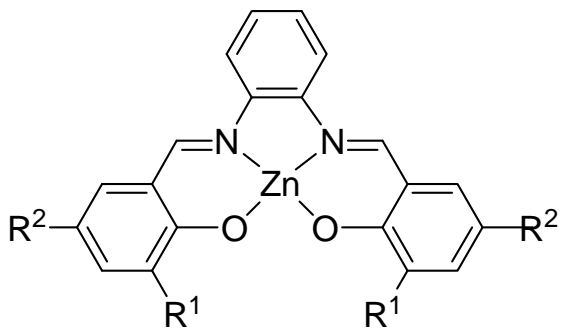

$103 \mathrm{R}^{1}=\mathrm{tBu}, \mathrm{R}^{2}=\mathrm{tBu}$

$104 \mathrm{R}^{1}=\mathrm{DMA}, \mathrm{R}^{2}=\mathrm{tBu}$

$105 \mathrm{R}^{1}=\mathrm{H}, \mathrm{R}^{2}=\mathrm{tBu}$

$106 \mathrm{R}^{1}=\mathrm{H}, \mathrm{R}^{2}=\mathrm{Cl}$

$107 \mathrm{R}^{1}=\mathrm{H}, \mathrm{R}^{2}=\mathrm{NO}_{2}$

$108 \mathrm{R}^{1}=\mathrm{H}, \mathrm{R}^{2}=\mathrm{H}$

$109 \mathrm{R}^{1}=\mathrm{H}, \mathrm{R}^{2}=\mathrm{OMe}$

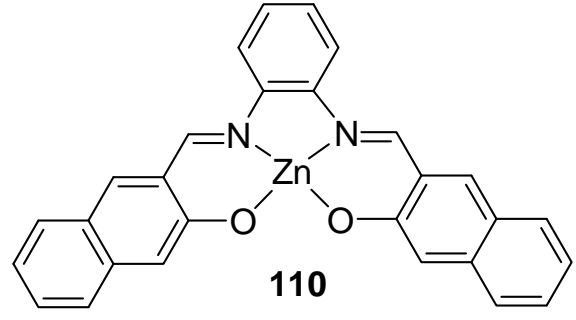

DMA = Dimethylamine

Fig. 52 Compounds 103-110

Knapp et al prepared Zn-salophen complex 103 (Fig. 52) and studied the fluorescent behaviour in the presence of nitroaromatic compounds and DMNB. ${ }^{99}$ The authors reported that addition of increasing quantities of DMNB, NX, NT, NB and NQ induced different quenching degrees of the emission band at $538 \mathrm{~nm}$ of the acetonitrile solutions of complex 103. Stern-Volmer plots indicated a dynamic quenching process in which nitro derivatives accepted an electron from the excited state of 103. In an additional work, the same authors prepared a family of $\mathrm{Zn}$ (salicylaldimine) derivatives (104-110). They were organised in a sensory array which was able to display differential responses to closely related nitroaromatics. ${ }^{100}$ The authors found that the emission of the $\mathrm{Zn}$ complexes was quenched to different extents upon the addition of nitro derivatives such as NX, 4-NT, NB, CINB, 2,4-DNT, 1,3-DNB, ClAmp, NQ and PA. In the score plot, the mononitroaromatic compounds were separated from not only dinitroaromatics, but also from ClAmp and PA. The emission quenching mechanism of complexes 104-110 by nitroaromatic derivatives was studied by means of emission lifetime measurements, EPR and DFT calculations. ${ }^{101}$

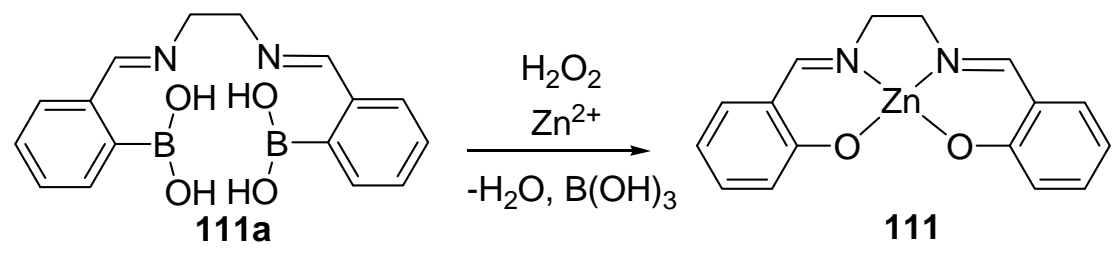

Fig. 53 Oxidative deboronation of the receptor 111a to obtain fluorescent 111 
The same group reported a highly sensitive system based on a turn-on fluorescence approach to detect low levels of $\mathrm{H}_{2} \mathrm{O}_{2}$ and $\mathrm{BP} .{ }^{102}$ The signalling protocol relied on an oxidative deboronation of a boronic acid derivative (111a) (Fig. 53), resulting in the formation of $\mathrm{H}_{2}$ Salen which is able to complex $\mathrm{Zn}^{2+}$ to form the corresponding $\mathrm{Zn}$ (Salen) fluorophore (111). Oxidative deboronation (see Figure 51) is chemically selective for peroxides and involved the conversion of a C-B bond into a $\mathrm{C}$ $\mathrm{O}$ bond by $\mathrm{H}_{2} \mathrm{O}_{2}$ and BP. The authors demonstrated that the methanolic solutions of 111a containing $\mathrm{Zn}(\mathrm{OAc})_{2}$ showed negligible fluorescence at $440 \mathrm{~nm}$, whereas the addition of increasing quantities of $\mathrm{H}_{2} \mathrm{O}_{2}$ induced the appearance of an intense emission band at $440 \mathrm{~nm}$ due to the formation of the highly emissive complex 111. The same response was observed in the presence of BP. In a parallel experiment, TATP was preincubated with acetic acid for 5 minutes and then added to 111a- $\mathrm{Zn}(\mathrm{OAc})_{2}$, the mixture resulting in an 80-fold increase of the emission intensity at $440 \mathrm{~nm}$. This emission enhancement was ascribed to the acid-promoted TATP decomposition that led to the formation of $\mathrm{H}_{2} \mathrm{O}_{2}$. The detection limits for $\mathrm{H}_{2} \mathrm{O}_{2}$, BP and TATP using this methodology were ca. $10 \mathrm{nM}$.

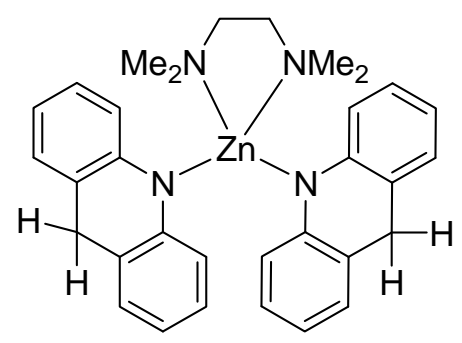

112

Fig. 54 Compound 112

A molecular fluorescence-based chemosensor for the direct detection of RDX and PETN was reported using the Zn complex 112 (Fig. 54). ${ }^{103}$ Acetonitrile solutions of 112 were weakly emissive, whereas the addition of RDX or PETN after a 30-second irradiation at $313 \mathrm{~nm}$ induced the appearance of an intense emission band at $480 \mathrm{~nm}$ (80- and 25-fold fluorescence enhancement at $480 \mathrm{~nm}$ for RDX and PETN, respectively). This emission was ascribed to the formation of an $N$-methylacridinium fluorophore $\left(\mathrm{AcrH}^{+}\right)$. The mechanism involved in the RDX and PETN photoreduction was extensively studied with analogue receptor 10-methyl-9,10-dihydroacridine $\left(\mathrm{AcrH}_{2}\right)$. The authors observed that RDX and PETN were able to oxidise $\mathrm{AcrH}_{2}$ by a photo-induced electron transfer mechanism, in which the photo-excited acridine 
$\left(\mathrm{AcrH}_{2}{ }^{*}\right)$ transferred one electron to $\mathrm{RDX}$, followed by hydride ion transfer to form the emissive $\mathrm{AcrH}^{+}$along with the decomposition products from RDX and PETN. Using complex 112, amounts as low as $7.0 \times 10^{-5}$ and $1.3 \times 10^{-4} \mathrm{~mol} \mathrm{dm}^{-3}$ of RDX and PETN, respectively, were detected.

\subsection{4.- Chemical reactions}

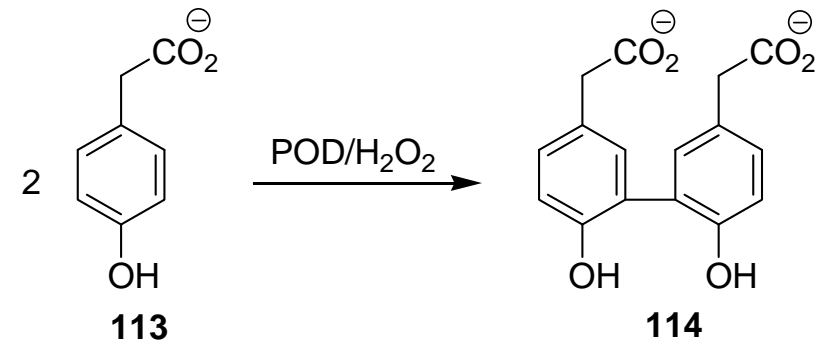

Fig.55 Formation of $\mathbf{1 1 4}$ by a peroxidase-catalysed oxidation of $\mathbf{1 1 3}$

The formation of fluorescent products by chemical reactions has been applied to the detection of peroxide-based explosives. For instance, Karst el al developed a reverse-phase HPLC with post-column UV irradiation and fluorescence detection for the determination of TATP and HMTD. ${ }^{104}$ After separation, analytes were degraded photochemically to hydrogen peroxide with UV light of $254 \mathrm{~nm}$. The hydrogen peroxide formed was determined through the formation of fluorescent dimer 114 (Fig. 55) (emission band at $405 \mathrm{~nm}$ ), which was obtained by the peroxidase-catalysed oxidation of $p$-hydroxyphenylacetic acid (113) (See Figure 53). The detection limits for both the peroxide-based explosives was $2.0 \times 10^{-6} \mathrm{~mol} \mathrm{dm}^{-3}$. The authors indicated that this method sufficed for the analysis of real samples, the analysis of an explosion site or to detect the remains of these two explosives, including illegal manufacturing sites. 


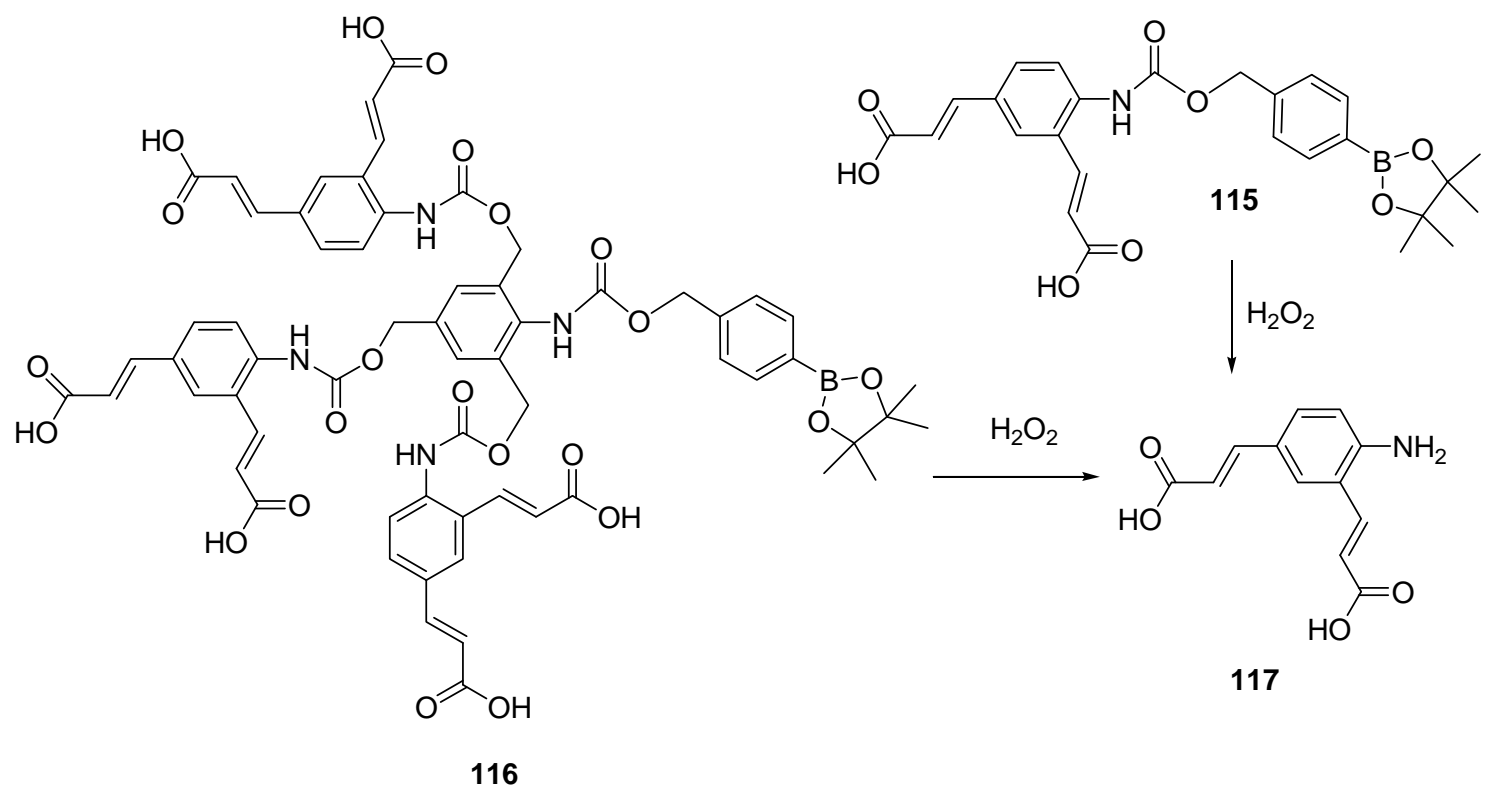

Fig. 56 TATP dendritic sensor 116 and its monomeric counterpart 115. The reaction with hydrogen peroxide (from TATP decomposition) induced the release of fluorescent amine 117.

Shabat et al reported a new molecular probe for the sensitive, direct detection of TATP in microgram amounts in aqueous buffer solutions with no acid pretreatment. It is based on a self-immolative dendritic platform that can amplify a single cleavage event generated by hydrogen peroxide into a multiple release of fluorogenic endgroups. ${ }^{105}$ For this purpose, probes 115 and 116 (Fig. 56) were prepared and tested. Both derivatives contained strongly fluorescent amine 117, connected to an aryl borate ester through carbamate linkages. Final probes 115 and 116 showed an intense emission band centred at $450 \mathrm{~nm}$ upon excitation at $270 \mathrm{~nm}$ in water containing $\mathrm{NaHCO}_{3}$ at $\mathrm{pH} 8.3$. After a 90-min incubation, addition of hydrogen peroxide to the solutions of both probes led to the appearance of a new emission band at $510 \mathrm{~nm}$, which was assigned to free 117. The release of $\mathbf{1 1 7}$ from the aqueous solutions of $\mathbf{1 1 5}$ and $\mathbf{1 1 6}$ was a direct consequence of the mechanism shown in Figure 54. Hydrogen peroxide was easily detected at a concentration of $1.0 \times 10^{-6} \mathrm{~mol} \mathrm{dm}^{-3}$. As expected, the intensity of the fluorescence generated by dendritic probe $\mathbf{1 1 6}$ in the presence of hydrogen peroxide was 3-fold higher than that obtained for 115. The same response was observed after a 120min incubation of both probes in the presence of TATP. 


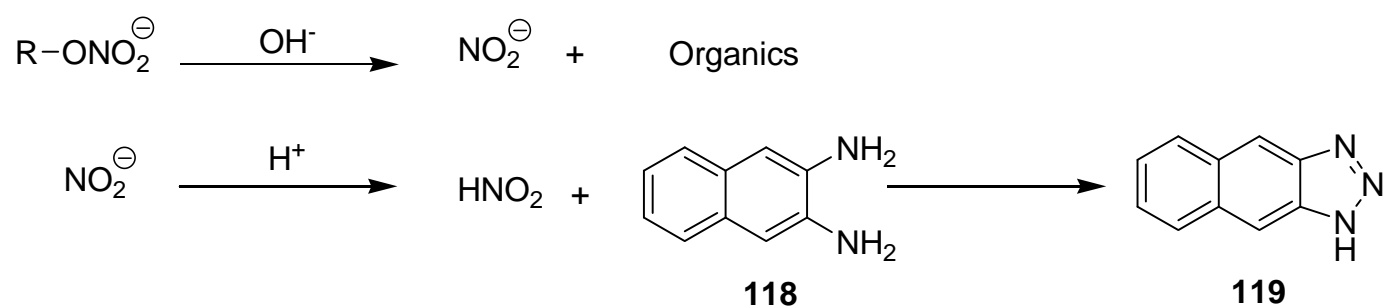

Fig. 57 Reaction scheme for steps 1 and 2 of two-step

detection process to obtain $\mathbf{1 1 9}$

Trogler et al developed a protocol for the detection of TNT, RDX, HMX, PETN, Tetryl, and TNG in the solid state. ${ }^{106}$ These authors prepared three reactives: reactive A, containing a polysilole polymer dissolved in acetone; reactive $\mathrm{B}$, formed by $2,3-$ diaminonaphthalene (DAN) and potassium hydroxide; and finally reactive C, a mixture of ethanol and phosphoric acid. Nitramine and nitrate esters were detected by a two-step procedure using reactives $\mathrm{B}$ and $\mathrm{C}$ (Fig. 57). Thus when applying reactive $\mathrm{B}$ to a filter paper containing solid explosives, the base contained in the mixture deprotonated either the nitramine or the nitrate esters in the alpha position to $\mathrm{N}$ or $\mathrm{O}$, respectively. This deprotonation induced the release of a nitrite anion. In the second step, upon application of reactive $\mathrm{C}$, the nitrite anion was converted into nitrous acid which induced the transformation of $\mathbf{1 1 8}$ into the highly fluorescent 1-[H]-naphthotriazole 119. With this two-step protocol, TNT gave no response, but nitramine and nitrate esters were detected at quantities as low as $2 \mathrm{ng}$. Additionally, the authors incorporated reactive A, which was able to detect TNT via the quenching of the polysilole polymer.

\subsection{5.- Miscellaneous}<smiles>O=C1c2ccccc2C(=O)c2c(O)c(O)cc(O)c21</smiles>

120

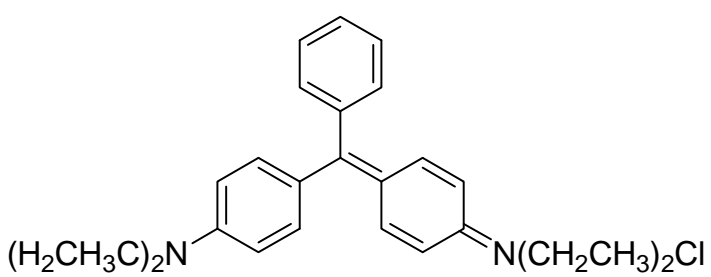

121<smiles>O=C1C=CC(=C(c2ccc(O)cc2)c2ccccc2S(=O)(=O)O)C=C1</smiles>

122

Fig. 58 Compounds 120-122 
Detection of target nitrated compounds (NM, NB, 4-NT and 2,6-DNT) by using common fluorophores purpurin (120), malachite green (121), and phenol red (122) (Fig. 58) has been studied. ${ }^{107}$ Addition of all the nitrated derivatives to ethanolic solutions of the three fluorophores induced different degrees of emission quenching. For the three fluorophores, the Stern-Volmer constants measured for nitroaromatic derivatives were higher than those determined for the nitroaliphatic counterparts. Of the three fluorophores, purpurin showed the greatest quenching constants. As in similar cases, the emission quenching of the three fluorophores was ascribed to a photo-induced electron transfer from the excited state of fluorophores to the ground state of nitro derivatives.

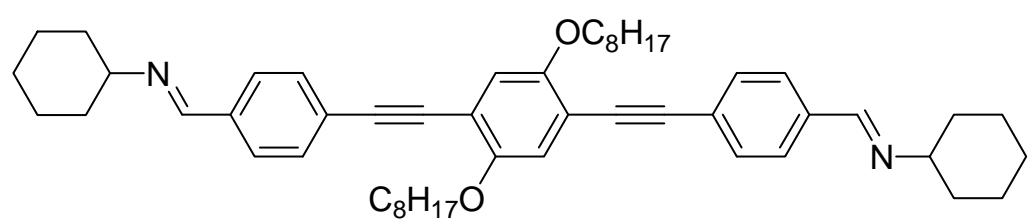

123

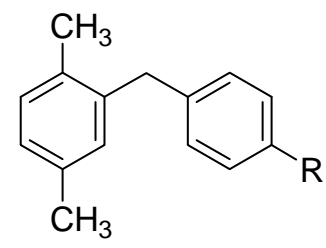

$$
\begin{aligned}
& 124 \mathrm{R}=-\mathrm{H} \\
& 125 \mathrm{R}=-\mathrm{CH}_{3} \\
& 126 \mathrm{R}=-\mathrm{OC}(\mathrm{O}) \mathrm{CH}_{3} \\
& 127 \mathrm{R}=-\mathrm{OCH}_{3} \\
& 128 \mathrm{R}=-\mathrm{OH}
\end{aligned}
$$

Fig. 59 Compounds 123-128

Montméat et al described a portable, low-cost and reliable detection device based on a thin film of the fluorescent $\pi$-conjugated phenylene-ethylene diimine 123 (Fig. 59). The film was deposited on a glass substrate, then acted as a wave-guide, and was used for the ultra-trace detection of TNT or 2,4-DNT in ambient air or on objects tainted with these explosives. ${ }^{108}$ This material exhibited excellent fluorescence properties and the high electron density of the diimine conferred it a strong affinity to electron-deficient compounds such as nitroaromatics. The film's fluorescence (greenish-blue fluorescence with a maximum at $480 \mathrm{~nm}$ ) diminished in the presence of either TNT (40\% of quenching after 10 minutes) or 2,4-DNT ( $80 \%$ of quenching after 10 minutes). The film of 123 presented detection thresholds of $0.75 \mathrm{ppbv}$ and $9 \mathrm{ppbv}$ for TNT and 2,4-DNT, respectively, with no loss of performance in the presence of humidity or interfering compounds.

2,5-dimethylphenyl-(4-substituted-phenyl)methanes (124-128) were synthesised by Cartmill et al and their ability to recognise nitroaromatic compounds in solution was 
tested. ${ }^{109}$ The hexane-ethyl acetate $91: 9 \mathrm{v} / \mathrm{v}$ solutions of receptors 124-128 showed emission bands in the 400-440 $\mathrm{nm}$ interval, which were quenched upon the addition of 2,4-DNT, 4-NT and NB. Emission quenching was assigned to an efficient photoinduced electron transfer between the excited receptor and the nitroaromatic derivative upon the formation of 1:1 complexes. The larger quenching was observed for receptor 124 in the presence of 2,4-DNT.

\section{3.- Colorimetric sensors and reagents for explosives.}

\section{1.- Colour changes induced by coordination.}

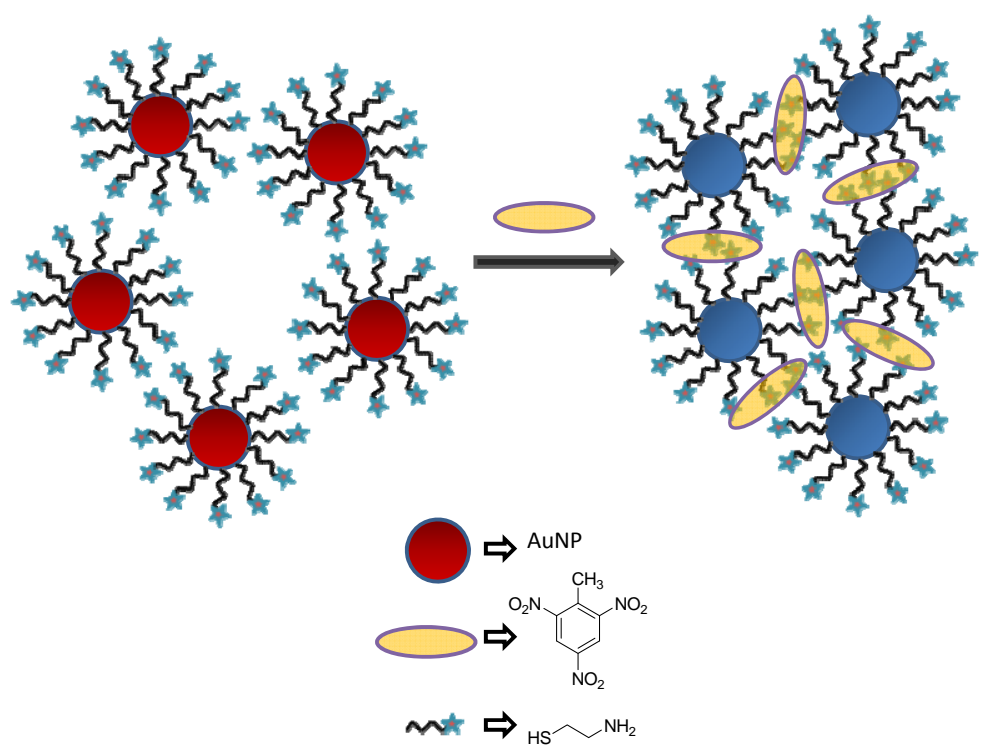

Fig. 60 Scheme of the colorimetric sensing of TNT based on the aggregation of functionalised-gold nanoparticles.

Mao et al developed a simple yet sensitive method based on the colour change of cysteamine-functionalised AuNPs, which went from red wine (dispersion state) to violet blue (aggregation state) induced by the donor-acceptor (D-A) interaction between TNT and primary amines (Fig. 60). ${ }^{110}$ The detection limit achieved was lower than picomolar, and the authors reported a gradual change in colour at different TNT concentrations ranging from $5 \cdot 10^{-13} \mathrm{M}$ to $5 \cdot 10^{-9} \mathrm{M}$ in aqueous environments. The presence of other explosives and derivates such as 2,4-dinitrotoluene, nitrobenzene or toluene did not induce changes in either colour or the absorbance spectrum of the AuNPs suspension. 


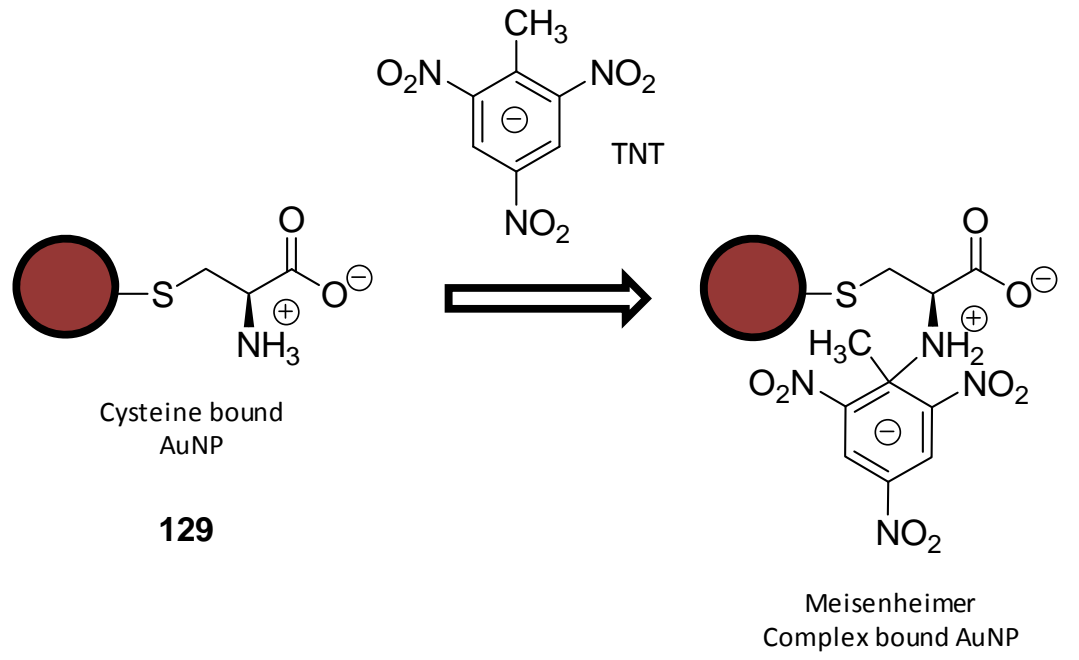

Fig. 61 Scheme of the formation of the Meisenheimer complex between TNT and cysteine-conjugated AuNPs

A highly selective and ultrasensitive cysteine-modified gold nanoparticle labelfree surface enhanced Raman spectroscopy (SERS) probe for TNT recognition at the pico molar (pM) level in aqueous solution was reported by Ray et al. ${ }^{111}$ Due to the formation of the Meisenheimer complex between TNT and cysteine-conjugated AuNPs (129), a substantial shift in plasmon band energy to a longer wavelength and a red-toblue colour change were observed (Fig. 61). As a result of this aggregation process, the intensity of the Raman signals significantly enhanced and attributed to the plasmonic coupling between nanoparticles in close proximity, resulting in large local electromagnetic field enhancements in the confined junctions. By this method, TNT can be detected quickly (in less than $10 \mathrm{~min}$ ) and accurately without any dye tagging at the pM level with excellent discrimination against other nitro compounds such as 2,4-DNT, NP, NB and heavy metals.

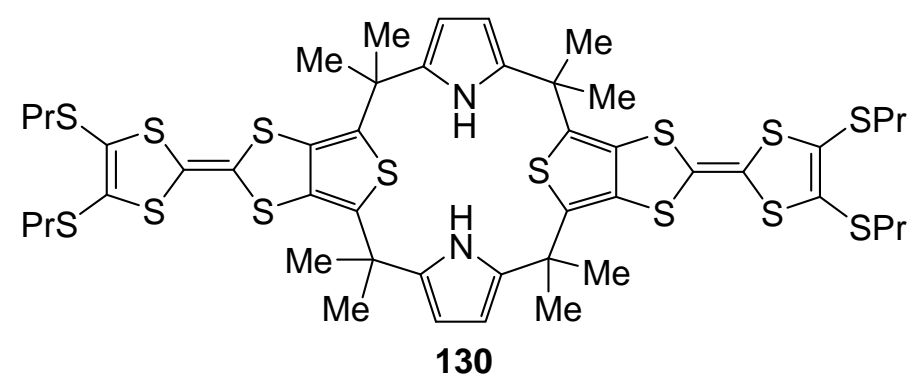

Fig. 62 Compound 130 
A colorimetric chemosensor based on bis-tetrathiafluvalene-calix[2]thiophene[2] pyrrole derivative 130 (Fig. 62) was synthesised and its chromogenic behaviour against several nitroaromatic explosives (TNT, PA and TNB) was tested. ${ }^{112}$ The chloroform solutions of 130 showed an intense absorption band at $450 \mathrm{~nm}$. Addition of TNB to the chloroform solutions of $\mathbf{1 3 0}$ resulted in the appearance of a small band at $649 \mathrm{~nm}$ with a clear colour change from yellow to green, whereas addition of PA led to another absorption at $746 \mathrm{~nm}$ (colour change from yellow to dark green). In contrast, addition of TNT induced negligible changes in the UV-visible spectra of 130. The colour changes observed in the presence of TNB and PA were ascribed to the formation of chargetransfer complexes between 130 and nitroaromatic molecules. In these complexes, nitroaromatic molecules were located between both tetrathiafulvalene (TTF) subunits in a sandwich-like fashion with $\pi-\pi$ donor-acceptor interactions. Hydrogen bonding interactions between the pyrrolic N-H protons of $\mathbf{1 3 0}$ and the oxygen atoms of the nitro groups (and the hydroxyl group in the case of PA) were also present. Finally, the authors demonstrated that addition of the chloroform solutions of receptor $\mathbf{1 3 0}$ to silica gel containing TNB, PA and TNT also led to a chromogenic response.

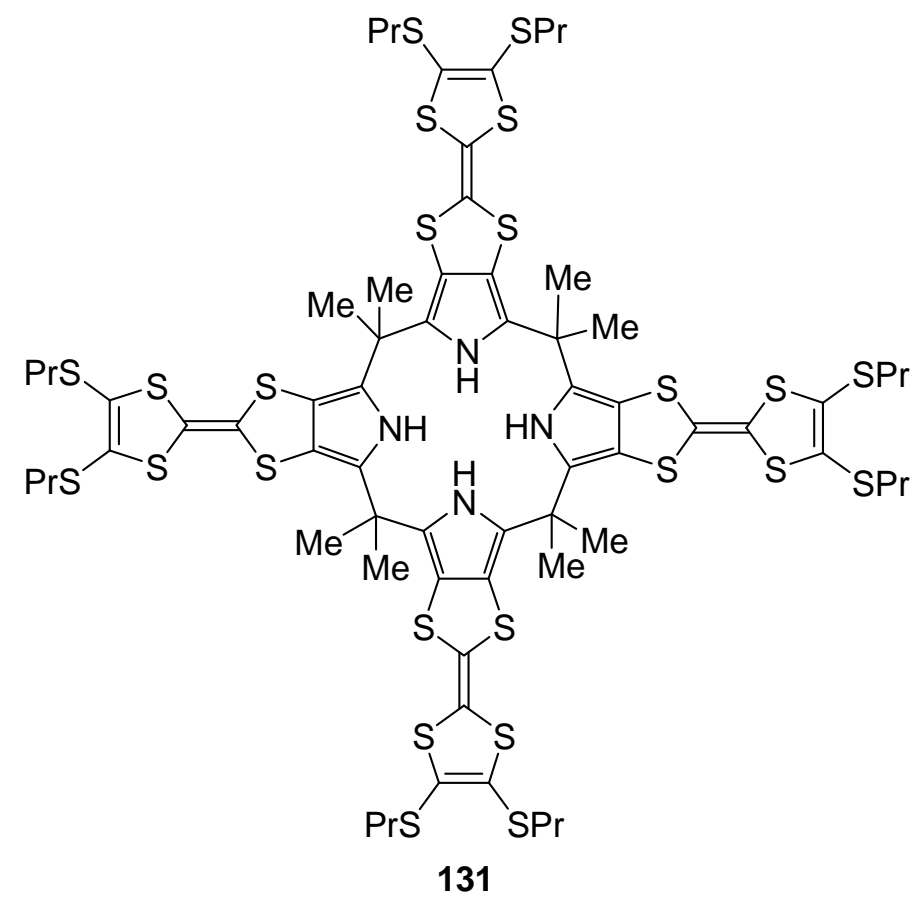

Fig. 63 Compound 131

The same authors designed a calix-[4]pyrrole derivative by incorporating four appended TTF units (131) (Fig. 63) which acted as a sandwich-like host for the electron-deficient guests in its 1,3-alternate conformation via charge transfer 
interactions. ${ }^{113}$ Receptor $\mathbf{1 3 1}$ acted as an effective receptor for molecules, such as TNB, tetrafluoro-p-benzoquinone, tetrachloro-p-benzoquinone and $p$-benzoquinone, in $\mathrm{CH}_{2} \mathrm{Cl}_{2}$ solutions. Receptor 131 showed a yellow colouration in dichloromethane, which changed to green with the addition of 2 equivalents of TNB given the appearance of a band at $677 \mathrm{~nm}$ resulting from the formation of the 131.(TNB) complex, in which TNB molecules were sandwiched between each pair of TTF arms. Addition of chloride anions to the dichloromethane solutions of the 131 $(\mathrm{TNB})_{2}$ complex brought about a colour change from green to yellow due to the binding of this anion with receptor 131, which adopted cone conformation. Finally, the same colour change was obtained upon the addition of tetrafluoro- $p$-benzoquinone, tetrachloro- $p$ benzoquinone and p-benzoquinone to the dicholomethane solutions of $\mathbf{1 3 1 .}$

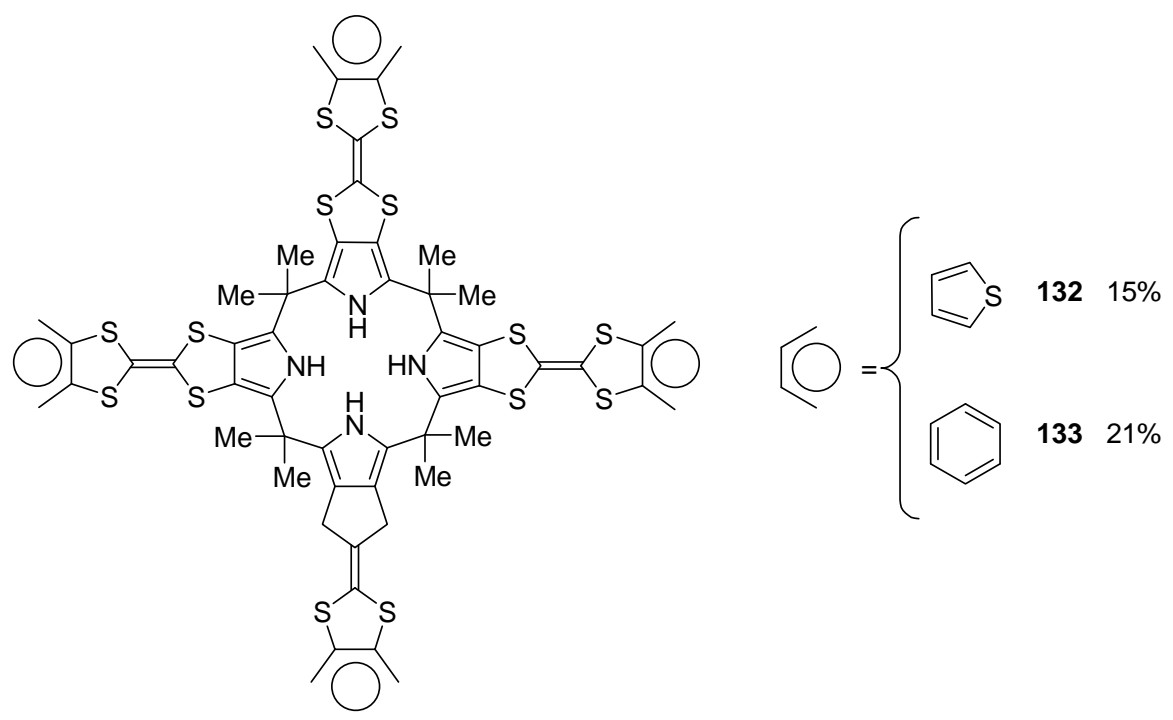

Fig. 64 Compounds 132 and 133

In another work Jeppesen, Sessler et al reported selective colorimetric sensing probes for nitroaromatic guests (TNB, TNT and PA) based on the use of modified tetrathiafulvalene (TTF)-calix[4]pyrroles $\left(\mathbf{1 3 1}, \mathbf{1 3 2}\right.$, 133) (Fig. 64) frameworks. ${ }^{114}$ As cited above, the chromogenic behaviour of receptor 131 in the presence of TNB was similar to that observed in the presence of TNT and PA in the chloroform solutions of this compound (colour modulations from yellow to green). In contrast, receptor 131 was characterised by modest binding affinities to TNB, TNT and PA in organic solvents, and was also found to require relatively high concentrations to produce a detectable colorimetric response to the naked eye. Almost the same results, but with better sensitivities, were obtained with receptors 132 and 133 upon binding with TNB, TNT 
and PA. For instance, the yellow chloroform solutions of receptors 132 and 133 changed to dark green when TNB was added, and to green upon the addition of TNT and PA. Changes in colour were ascribed to the formation of charge-transfer complexes between the receptors in the 1,3-alternate conformation and nitroaromatic molecules. In all cases, 1:2 receptor-nitroaromatic complexes were observed in which explosives were located in the TTF subunits on the opposite sides of the central calix[4]pyrrole core. The greatest affinity was observed for PA $(\mathrm{PA}>\mathrm{TNB}>\mathrm{TNT})$. The detection limits observed were; TNB: 0.44, 0.30, $1.56 \mu \mathrm{gmL}^{-1}$; PA: 0.77, 0.64, $2.68 \mu \mathrm{gmL}^{-1}$; TNT: 3.04 , $2.72,15.3 \mu \mathrm{gmL}^{-1}$, for receptors $\mathbf{1 3 3}, \mathbf{1 3 2}$, and $\mathbf{1 3 1}$, respectively.

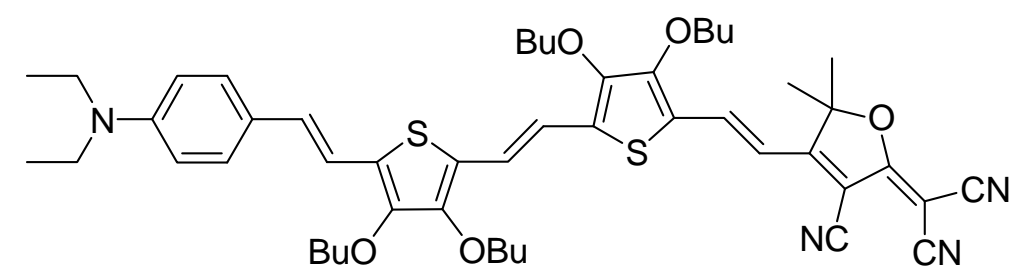

134
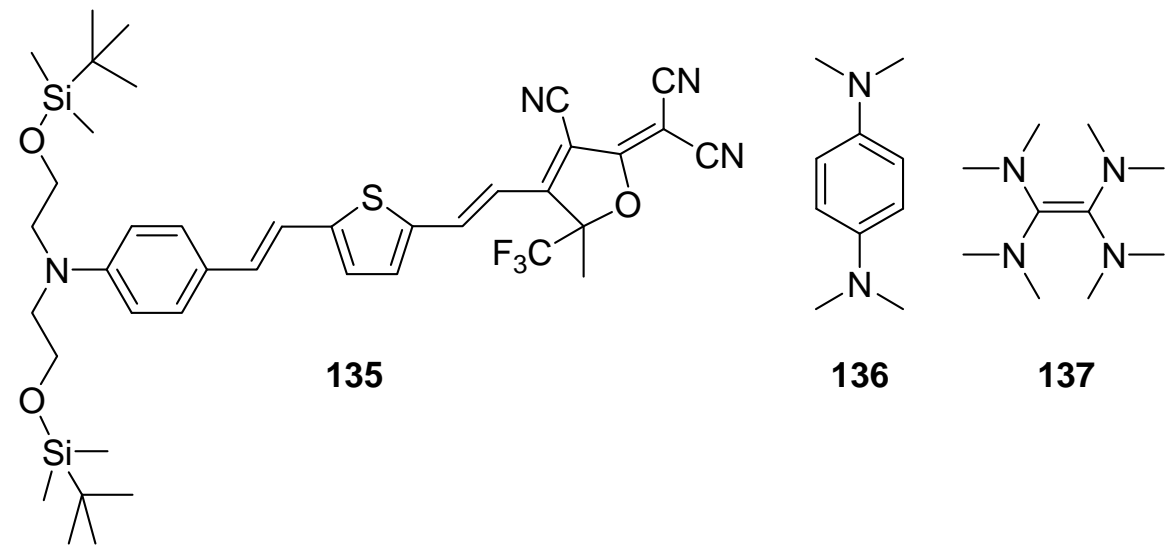

Fig. 65 Compounds 134-137

The reaction between cymantrene (cyclopentadienylmanganesetricarbonyl) and nitroaromatics upon UV irradiation (330-350 nm) to yield a blue colour derivative (absorption band at $680 \mathrm{~nm}$ ) was used to detect explosives. ${ }^{115}$ The thin films of divinylstyrene copolymer were embedded with cymantrene (10-40\%) and used for the colorimetric detection and quantification of 2,4-DNT, 1,3-DNB and 2-NT (a volatile taggant of TNT). The response obtained in the presence of 2,4-DNT, 1,3-DNB and 2NT was nearly the same with detection limits of $0.2 \mathrm{ng}$ for nitroaromatic derivatives.

Dyes 134 and 135 (Fig. 65), included in polymeric thin films and spin-coated on glass slides, were used for the colorimetric detection of $2,4-\mathrm{DNT} .{ }^{116}$ For instance, the 
thin films of poly(methylmetacrylate) (PMMA) containing 134 showed an absorption band at $630 \mathrm{~nm}$, which underwent a red shift and a reduction in absorbance upon exposure ( 24 hours) to air saturated with $2,4-\mathrm{DNT}$ at $65^{\circ} \mathrm{C}$. This was reflected by a change in colour from dark blue to light purple. A similar trend was observed when exposing the thin films of amorphous polycarbonate (APC) containing 135 to 2,4-DNT vapours at $65^{\circ} \mathrm{C}$. Optical changes were ascribed to the formation of a charge-transfer complex between 134, 135 and the nitroaromatic molecules. Apart from changes in colour, exposure of chromophore-containing films to 2,4-DNT vapours induced important changes in the refraction index of polymers. Finally, chromophore-containing polymers were used for the construction of microring resonators, which allowed the detection of 2,4-DNT at the ppb level.

A novel and simple method for the colorimetric detection and differentiation of nitro explosives by specific colour reactions using host-guest and charge-transfer acceptor/donor complexes has been developed. ${ }^{117}$ Different colours were observed when several charge-transfer complexes were formed using nitrated explosives (TNT, Tetryl, RDX and HMX) as electron acceptors and electron donor molecules; for example, $N, N, N$ ', $N$ '-tetramethyl-p-phenylenediamine (136) and tetrakis(dimethylamino) ethylene (137) in the presence or absence of $\gamma$-cyclodextrin $(\gamma-C D)$. For instance, 136 and TNT formed a charge-transfer complex in acetonitrile (absorption band at $504 \mathrm{~nm}$ ). When changing to a more competitive solvent, such as acetonitrile-water 95:5 v/v, the band at $504 \mathrm{~nm}$ disappeared and two new absorptions emerged at 560 and $610 \mathrm{~nm}$ (the solution became violet). This colour change was attributed to the rupture of the charge-transfer complex and to the formation of radicals $\mathrm{TNT}^{\bullet-}$ and $\mathbf{1 3 6}^{\mathbf{\bullet}}$. When the same experiments were carried out in the presence of $\gamma-\mathrm{CD}$ (acetonitrile-water 95:5 v/v), the chargetransfer complex between 136 and TNT again formed in the inner hydrophobic cavity of the macrocycle. Almost the same response in acetonitrile was observed by changing TNT to RDX, but in this case, the formed complex showed a very weak absorbance at $504 \mathrm{~nm}$. Once again, addition of water induced the formation of the corresponding radicals. However in this case, that is, in the presence of $\gamma-\mathrm{CD}$, the charge-transfer complexes did not form. This different chromogenic behaviour of TNT and RDX in the presence of $\gamma-\mathrm{CD}$ was the basis of a pattern colorimetric recognition of nitrated explosives via a sensor array design. Electron donor 137 was also used for the development of a sensing array with 136 without the use of $\gamma$-CD. Similar discrimination features were observed. 


\section{2.- Colour changes induced by chemical reactions.}

The use of chemical reactions which induced the formation of coloured or fluorescent compounds to recognise nitroaromatic explosives has been extensively employed. In fact, the first examples had already been described in the last decade of the $19^{\text {th }}$ century. In line with this, Janowski's reaction provided an adequate test for dinitro and trinitro aromatic compounds by means of the characteristic colour developed in alkaline acetone. ${ }^{118}$ In 1948, English described enhanced results in sensitivity terms using 50/50 acetone-ethanol mixtures and potassium hydroxide as a base. ${ }^{119}$ Some years later, Amas and Yallop proposed the use of different organic bases, such as tetramethylammonium hydroxide, diethylene triamine and tetraethylene pentamine, to induce the colorimetric reactions in the presence of 2,4-DNT and TNT at low concentrations. ${ }^{120}$ For instance, addition of tetramethylammonium hydroxide to acetoneethanol solutions of 2,4-DNT resulted in the formation of a blue colour (detection limit of $2 \mu \mathrm{g}$ ), whereas a red colour was observed with TNT (detection limit of $1 \mu \mathrm{g}$ ). Furthermore, a slightly yellow colour developed with TNG. The same authors described a specific test for RDX based on the appearance of a red colour achieved in the presence of thymol and nitrogen-free sulphuric acid. However, this colour was also induced by other compounds like sugars and aldehydes. ${ }^{121}$ Three years later, the same authors improved the colorimetric method to eliminate interferences. ${ }^{122}$

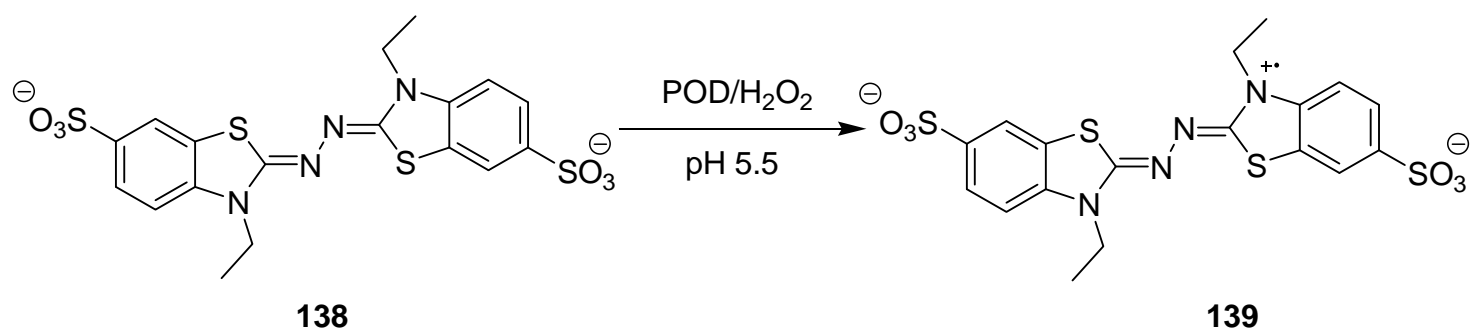

Fig. 66 Enzymatic reaction between POD and $\mathbf{1 3 8}$ which yields the green-coloured radical cation 139

The recent literature contains examples of using the chemodosimeter approach to design colorimetric probes, which have been reported mainly for the detection of peroxide-based explosives. For instance, Karst et al reported the development of a new rapid, simple field test for the chromogenic detection of peroxide-based explosives such as TATP and HMTD. ${ }^{123}$ The proposed method uses decomposition of peroxide-based 
explosives via UV irradiation $(254 \mathrm{~nm})$ to yield hydrogen peroxide, which is further determined by an enzymatic reaction between horseradish peroxidase (POD) and substrate 138 (2,2'-azino-bis(3-ethylbenzothiazoline)-6-sulphonate), which yields the green-coloured radical cation 139 in acetonitrile-water 1:10 v/v mixtures (Fig. 66). Detection limits of $8.0 \times 10^{-6} \mathrm{~mol} \mathrm{dm}^{-3}$ and $8.0 \times 10^{-7} \mathrm{~mol} \mathrm{dm}{ }^{-3}$ were determined for TATP and HMTD, respectively. In order to obtain lower detection limits, the same enzymatic assay was carried out with 113 (p-hydroxyphenylacetic acid), which dimerises to form the highly fluorescent compound 114 (emission band at $405 \mathrm{~nm}$ upon excitation at $320 \mathrm{~nm}$ ). Using this fluorescence-based test, limits of detection of $8.0 \times 10^{-}$ ${ }^{7} \mathrm{~mol} \mathrm{dm}^{-3}$ were obtained for both TATP and HMTD.

Apak et al described a procedure for the on-site colorimetric signalling of TATP and HMTD based on the detection of $\mathrm{H}_{2} \mathrm{O}_{2}$ formed upon hydrolysis via the reaction of hydrogen peroxide with the chromogenic reactive $\mathrm{Cu}(\mathrm{II})$-neocuproine, resulting in light absorption at $454 \mathrm{~nm}^{124}$ The hydrolysis reaction of TATP and HMTD was carried out in water-acetone 20:1 v/v mixtures. Using this simple procedure, the limit of detection was $0.2 \mathrm{mg} \mathrm{L}^{-1}$ for both TATP and HMTD. The method was successfully applied for the detection of TATP and HMTD without any interference by complex samples containing nitroexplosives, such as TNT, RDX and PETN, or by household detergents containing sodium percarbonate and perborate-based bleaching powder constituents. The procedure was also unaffected by the common ions present in soil extracts. Finally, and in order to enhance the applicability of the chromogenic method, the $\mathrm{Cu}(\mathrm{II})$-neocuproine complex was implemented in a polymeric nafion membrane to be used to accurately detect TATP and HMTD in the liquid phase.

Very recently, Suslick et al, developed a simple, yet highly sensitive colorimetric sensor array based on several redox sensitive dyes to detect TATP in the gas phase. $^{125}$ The authors pretreated a gas stream containing TATP vapours with Amberlyst-15 (an acid catalyst) with a view to inducing the decomposition of the peroxidic explosive to $\mathrm{H}_{2} \mathrm{O}_{2}$. Then the gas stream was passed through an array containing redox active dyes, which changed colour upon the reaction with hydrogen peroxide. Finally, the RGB coordinates of the developed colours were measured and colour difference maps were generated. By applying principal component analysis (PCA) to the data obtained from the array, TATP could be distinguished and determined (limit of detection of $2 \mathrm{ppb}$ ) from hydrogen peroxide, bleach, $t$-butylhydroperoxide and peracetic acid. 
A hybrid electrochemical-colorimetric sensor for the selective detection of nitroaromatic explosives in the gas phase has been recently published. ${ }^{126}$ The hybrid sensor is composed of a microcontroller-based potentiostat, a typical webcam, a transparent substrate of indium tin oxide (ITO)-printed electrodes, and a thin layer of 1butyl-3-methylimidazolium hexafluorophosphate $\left(\mathrm{BMIM}^{-} \mathrm{PF}_{6}\right)$ covering it. Upon the exposure of the sensor to the vapours of nitroaromatic explosives (PA, TNT, 2,4-DNT and 2-NT), an adsorption process in the BMIM-PF 6 layer occurred. This BMIM-PF 6 layer preconcentrated the explosives and quickly transported these analytes to electrodes. Application of a $-2.0 \mathrm{v}$ potential lasting 2.5 minutes induced the reduction of the nitroaromatic explosives to the corresponding azo and azoxy derivatives (red colour with absorbances at ca. $450 \mathrm{~nm}$ ). Changes in the RGB coordinates upon reduction of the nitroaromatic explosives were used for identification and quantification purposes. With this hybrid sensor, limits of detection of ppb in the vapour phase were achieved.

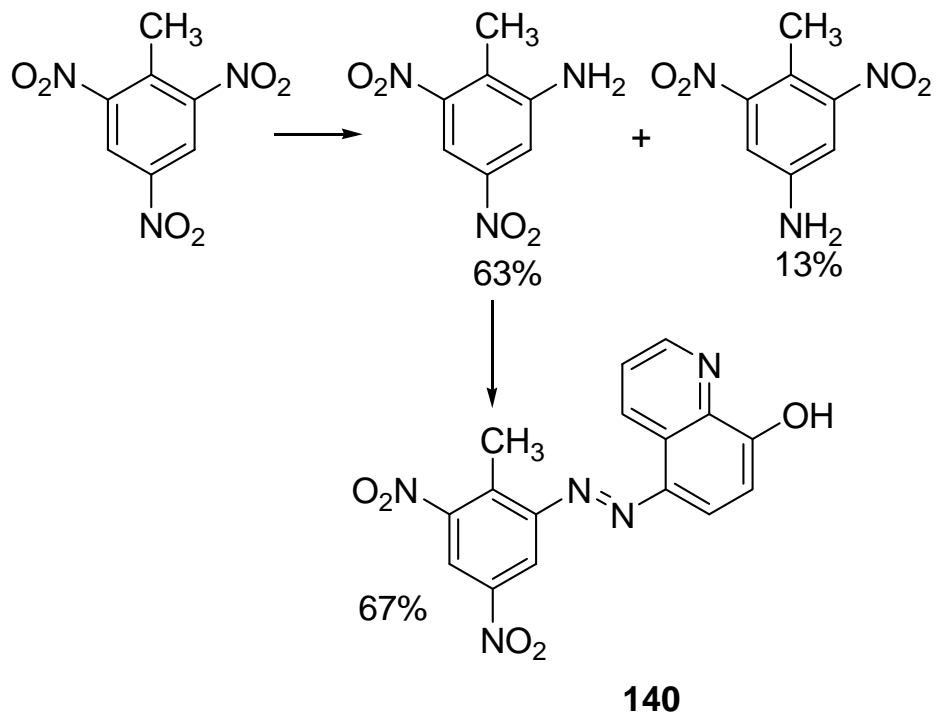

Fig. 67 Colorimetric recognition of TNT from the formation of an azoic dye $\mathbf{1 4 0}$

TNT was chemically modified to form an azo dye to be then easily detected by surface-enhanced resonance Raman scattering (SERRS). ${ }^{127}$ In this technique, an analyte containing a chromophore was adsorbed onto a roughened metal (usually silver or gold), and the enhanced Raman scattering from the surface was recorded. For this purpose, TNT was reduced to a mixture of 2-amino-4,6-dinitrotoluene $(63 \%)$ and 4 amino-2,6-dinitrotoluene (13\%) by using iron in acetic acid. Then, 2-amino-4,6dinitrotoluene was diazotised with 8-hydroxyquinoline to confer the coloured derivative 
140 (Fig. 67). An aqueous solution of 140 at $\mathrm{pH} 8.0$ showed an intense absorption band at $523 \mathrm{~nm}$ which could be used for the colorimetric recognition of TNT. Additionally, the SERRS spectrum of $\mathbf{1 4 0}$ was measured and detection limit as low as $1.0 \times 10^{-9} \mathrm{~mol}$ $\mathrm{dm}^{-3}$ was obtained.

Microchip capillary electrophoresis (CE) coupled with solid phase extraction (SPE) was used for the colorimetric detection of TNT, Tetryl and TNB in complex explosive mixtures containing TNT, Tetryl, TNB, 2-NT, 3-NT, 4-NT, NB, 1,3-DNB, 2,4-DNT, 2,6-DNT, 2-amino-4,6-DNT, HMX and RDX. ${ }^{128}$ Measurements were taken in acetonitrile-water 87.5-12.5 v/v mixtures containing $\mathrm{NaOH}(2.5 \mathrm{mM})$ and a surfactant (CTAB or SDS at the $0.25-1.5 \mathrm{mM}$ concentrations). Of all the explosives tested, only TNT, Tetryl and TNB were able to undergo the deprotonation process with bases leading to the formation of coloured products with bands in the 400-700 nm interval (TNT gave a purple colour, whereas Tetryl and TNB gave yellowish-brown solutions). In line with this, SPE was used to preconcentrate explosives from an aqueous sample into the mixture of organic solvents containing the base. Then, TNT, Tetryl and TNB formed the coloured deprotonated products, separated by CE. With this technique, trinitroaromatic explosives were detected at levels as low as $0.19-0.34 \mu \mathrm{g} \mathrm{L}^{-1}$ in water.

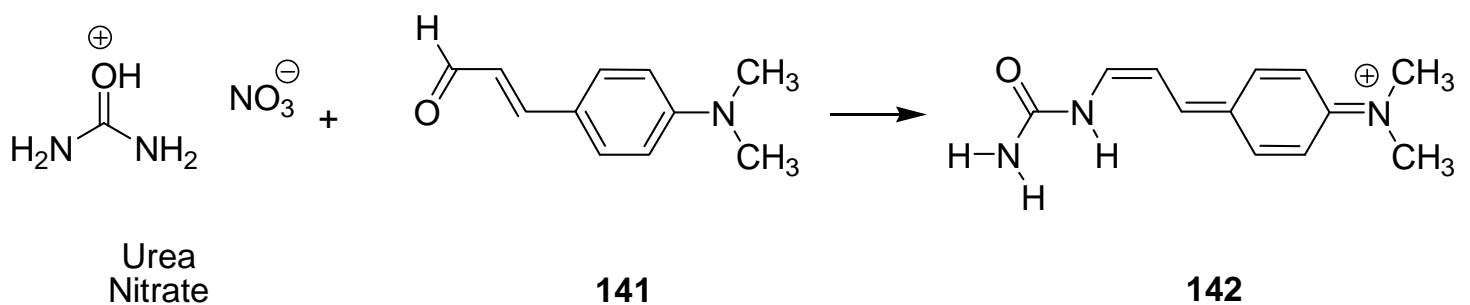

Fig. 68 Reaction between urea nitrate and $\mathbf{1 4 1}$ to yield the coloured derivative 142

A sensitive, specific and simple colour test for the improvised explosive urea nitrate was described. It was based on the formation of a coloured pigment upon the reaction between urea nitrate and p-dimethylaminocinnamaldehyde (141) (Fig. 68) under neutral conditions. ${ }^{129}$ The procedure involved the addition of urea nitrate (dissolved in water or in the solid form) to the ethanolic solutions of 141, resulting in the appearance of a red colour $\left(\lambda_{\max } 514 \mathrm{~nm}\right)$ due to the formation of compound 142. The detection limit on paper was about $100 \mu \mathrm{g} / \mathrm{cm}^{2}$. When dealing with possible interferences, urea itself does not react with 141 under the same conditions. Other potential sources of false-positive responses, such as acidic salts, with a $\mathrm{pH}$ lower than 
4 , brought about the formation of a light pink colour. The authors suggested that this reagent in neutral solution would provide a diagnostic test in trace amounts for the improvised explosive urea nitrate that could be used in the field, on hands of suspects, on door handles or in packaging devices, and with other suspected materials.

\section{4.- Conclusions and future perspectives}

In the last few decades, inspiration from several signalling paradigms has led to the development of chromo-fluorogenic probes for explosives with both enhanced functional behaviour and suitable selectivity and sensitivity. This review has been divided into two parts: one deals with fluorogenic systems, while the other relates to the use of chromogenic probes. Fluorogenic chemosensors have been more widely employed than chromogenic ones. Most reported examples using fluorescent signals have been reported for the detection of nitro explosives. In most cases, fluorogenic systems rely on a suitable interaction between electron-deficient nitro derivatives and electron-rich aromatic compounds. The latter are also highly fluorescent, whereas the former are efficient fluorescence quenchers through photo-induced electron transfer mechanisms. As a result of this design, a quenching process was observed as a signalling event in most of the systems reported for the detection of nitro explosives. As presented in Section 2.1, fluorescent-conjugated polymers have been employed extensively in recent years as sensing materials to detect explosives. One special feature of these systems is their ability to produce signal amplification in response to interactions with target analytes. Examples involving the use of poly(phenylene ethynylene)s, polyphenylene vinylenes and Si-containing polymers have been shown. Section 2.1 also includes a few examples that combine the appealing characteristics of imprinted polymers and the use of fluorescent signalling reporters, such as quantum dots or fluorescent-conjugated polymers which, at the same time, acted as a framework of the imprinted system and as a signalling reporter via quenching interactions. Coupling fluorescent functional chemical systems with inorganic supports to design hybrid signalling supports is a particularly fascinating area of research. This approach is relatively new; thus, it is not surprising that, despite the interesting examples reported in Section 2.2, the potential of hybrid signalling systems remains to be fully explored. Section 2.3 explores the use of single fluorophores as reporters. Polycyclic aromatic hydrocarbon and other fluorophores based on heterocycles have been fundamentally 
used to detect nitroaromatic explosives, whereas most of the examples following a chemodosimeter approach using irreversible chemical reactions have been designed to detect peroxide-based explosives. Finally, aggregation-induced colour changes, coordination interactions and the use of chemodosimeters have been used to develop chromogenic probes for nitro- and peroxide-based explosives. Yet despite these interesting examples, there are a low number of chromogenic probes for the selective and sensitive detection of target explosives, and this is still an unexplored area. In summary, the chemistry of chromo-fluorogenic probes for explosives is currently timely research within the field of sensing chemistry. Upcoming studies will probably involve the investigation of new signalling paradigms; for instance, using nano-hybrid systems, probes showing enhanced fluorescence instead of quenching upon interaction with explosives, coupling of probes with sophisticated instrumentation, and more in-depth research into the design of chromogenic chemosensors. A number of new results in this field is envisioned.

\section{Acknowledgments}

Financial support from the Spanish Government (project MAT2009-14564-C04) and the Generalitat Valencia (project PROMETEO/2009/016) is gratefully acknowledged.

\section{5.- References}

${ }^{1}$ K. G. Furton, L. J. Myers, Talanta, 2001, 54, 487-500.

${ }^{2}$ K. Hakansson, R. V. Coorey, R. A. Zubarev, V. L. Talrose, P. Hakansson, J. Mass Spectrom., 2000, 35, 337-346.

${ }^{3}$ M. E. Walsh, Talanta, 2001, 54, 427-438.

${ }^{4}$ J. M. Sylvia, J. A. Janni, J. D. Klein, K. M. Spencer, Anal. Chem., 2000, 72, 58345840 .

5 (a) J. Yinon, Mass Spectrom. Rev., 1982, 1, 257-307. (b) J. C. Mathurin, T. Faye, A. Brunot, J. C. Tabet, Anal. Chem., 2000, 72, 5055-5062.

${ }^{6}$ S. F. Hallowell, Talanta, 2001, 54, 447-458.

${ }^{7}$ C. Vourvopoulos, P. C. Womble, Talanta, 2001, 54, 459-468.

${ }^{8}$ M. Krausa, K. Schorb, J. Electroanal. Chem., 1999, 461, 10-13.

${ }^{9}$ (a) E. Wallis, T. M. Griffin, N. Popkie Jr., M. A. Eagan, R, F. McAtee, D. Vrazel, J. McKinly, Proc. SPIE-Int. Soc. Opt. Eng., 2005, 5795, 54-64. (b) G. A. Eiceman, J. A. Stone, Anal. Chem., 2004, 76, 390A-397A. 
${ }^{10}$ (a) J. L. Steinfeld, J. Wormhoudt, J. Annu. Rev. Phys. Chem., 1998, 49, 203-232. (b)

D. S. Moore, Rev. Sci. Instrum., 2004, 75, 2499-2512.

${ }^{11}$ R. Martínez-Máñez, F. Sancenón, M. Hecht, M. Biyical, K. Rurack, Anal. Bioanal. Chem., 2011, 399, 55-74.

12 (a) M. E. Moragues, R. Martínez-Máñez, F. Sancenón, Chem. Soc. Rev., 2011, 40, 2593-2643. (b) R. Martínez-Máñez, F. Sancenón, J. Fluoresc., 2005, 15, 267-285. (c) Z. Xu, X. Chen, H. N. Kim, J. Yoon, Chem. Soc. Rev., 2010, 39, 127-137.

${ }^{13}$ (a) E. M. Nolan, S. J. Lippard, Chem. Rev., 2008, 108, 3443-3480. (b) P. Pallavicini, Y. A. Díaz-Fernández, L. Pasotti, Coord. Chem. Rev., 2009, 253, 2226-2240. (c) E. L. Que, C. J. Chang, Chem. Soc. Rev., 2010, 39, 51-60.

${ }^{14}$ G. J. Mohr, Anal. Chim. Acta, 2004, 508, 233-237.

${ }^{15}$ R. Martínez-Máñez, F. Sancenón, Chem. Rev., 2003, 103, 4419-4476.

${ }^{16}$ J. P. Agrawal, R. D. Hodgson, Organic Chemistry of Explosives, John Wiley \& Sons, Chichester, 2007, ISBN-13 978-0-470-02967-1 (HB).

${ }^{17}$ C. J. Cumming, C. Aker, M. Fisher, M. Fox, M. J. la Grone, D. Reust, M. G. Rockley, T. M. Swager, E. Towers, V. Williams, IEEE Transactions on Geoscience and Remote Sensing, 2001, 39, 1119-1128.

${ }^{18}$ S. J. Toal, W. C. Trogler, J. Mater. Chem., 2006, 16, 2871-2883.

${ }^{19}$ D.T. McQuade, A.E. Pullen, T.M. Swager, Chem. Rev. 2000, 100, 2537-2574.

${ }^{20}$ D.T. McQuade, A.E. Pullen, T.M. Swager, Chem. Rev. 2000, 100, 2537-2574.

${ }^{21}$ Q. Zhou, T. M: Swager, J. Am. Chem. Soc., 1995, 117, 7017-7018.

${ }^{22}$ J. -S. Yang, T. M. Swager, J. Am. Chem. Soc., 1998, 120, 5321-5322.

${ }^{23}$ J. -S. Yang, T. M. Swager, J. Am. Chem. Soc., 1998, 120, 11864-11873.

${ }^{24}$ S. Yamaguchi, T. M. Swager, J. Am. Chem. Soc., 2001, 123, 12087-12088.

${ }^{25}$ S. Zahn, T. M. Swager, Angew. Chem. Int. Ed., 2002, 41, 4226-4230.

${ }^{26}$ J. P. Amara, T. M. Swager, Macromolecules, 2005, 38, 9091-9094.

${ }^{27}$ D. Zhao, T. M. Swager, Macromolecules, 2005, 38, 9377-9384.

${ }^{28}$ S. W. Thomas III, J. P. Amara, R. E. Bjork, T. M. Swager, Chem. Commun., 2005, $4572-4574$.

${ }^{29}$ A. Narayanan, O. P. Varnavsky, T. M. Swager, T. Goodson III, J. Phys. Chem. C, 2008, 112, 881-884.

${ }^{30}$ S. Chen, Q. Zhang, J. Zhang, J. Gu, L. Zhang, Sensors Act. B, 2010, 149, 155-160.

31 Y. Long, H. Chen, Y. Yang, H. Wang, Y. Yang, N. Li, K. LI, J. Pei, F. Liu, Macromolecules, 2009, 42, 6501-6509. 
${ }^{32}$ C. -P. Chang, C. -Y. Chao, J. H. Huang, A. -K. Li, C. -S. Hsu, M. -S. Lin, B. R. Hsieh, A. -C. Su, Synth. Met., 2004, 144, 297-301.

${ }^{33}$ I.A. Levitsky, W.B. Euler, N. Tokranova, A. Rose, Appl. Phys. Lett., 2007, 90, 041904.

${ }^{34}$ L. Chem, D. McBranch, R. Wang, D. Whitten, Chem. Phys. Lett., 2000, 330, 27-33.

${ }^{35}$ A. Rose, Z. Zhu, C.F. Madigan, T.M. Swager, V. Bulovic, Nature, 2005, 434, 876879.

${ }^{36}$ K. Tamao, M. Uchida, T. Izumizawa, K. Furukawa, S. Yamaguchi, J. Am. Chem. Soc., 1996, 118, 11974-11975.

${ }^{37}$ H. Sohn, R. R. Huddleston, D. R. Powell, R. West, J. Am. Chem. Soc., 1999, 121, 2935-2936.

${ }^{38}$ H. Sohn, R. M. Calhoun, M. J. Sailor, W. C. Trogler, Angew. Chem. Int. Ed., 2001, 40, 2104-2105.

39 J. Ohshita, A. Kunai, Acta Polym., 1998, 49, 379-403.

${ }^{40}$ H. Sohn, R. M. Calhoun, M. J. Sailor, W. C. Trogler, Angew. Chem. Int. Ed., 2001, 40, 2104-2105.

${ }^{41}$ S. J. Toal, D. Magde, W. C. Trogler, Chem. Commun., 2005, 5465-5467.

${ }^{42}$ H. Sohn, M. J. Sailor, D. Magde, W. C. Trogler, J. Am. Chem. Soc., 2003, 125, 38213830 .

43 J. C. Sanchez. A. G. DiPascuale, A. L. Rheingold, W. C. Trogler, Chem. Mater., 2007, 19, 6459-6470.

${ }^{44}$ J. C. Sanchez, S. A. Urbas, S. J. Toal A. G. DiPascuale, A. L. Rheingold, W. C. Trogler, Macromolecules, 2008, 41, 1237-1245.

45 J. C. Sanchez, W. C. Trogler, J. Mater. Chem., 2008, 18, 3143-3156.

${ }^{46}$ J. Liu, Y. Zhong, J. W. Y. Lam, P. Lu, Y. Hong, Y. Yu, Y. Yue, M. Faisal, H. H. Y. Sung, I. D. Williams, K. S. Wong, B. Z. Tang, Macromolecules, 2010, 43, 4921-4936.

${ }^{47}$ P. Lu, J. W. Y. Lam, J. Liu, C. K. W. Jim, W. Yuan, N. Xie, Y. Zhong, Q. Hu, K. S. Wong, K. K. L. Cheuk, B. Z. Tang, Macromol. Rapid Commun., 2010, 31, 834-839.

${ }^{48}$ Y. Liu, R. C. Mills, J. M. Boncella, K. S. Schanze, Langmuir, 2001, 17, 7452-7455.

${ }^{49}$ L. G. Toy, K. Nagai, B. D. Freeman, I. Pinnau, T. Masuda, M. Teraguchi, Y. P. Yampolskii, Macromolecules, 2000, 33, 2516-2524.

${ }^{50}$ (a) A. Saxena, M. Fujiki, R. Rai, G. Kwak, Chem. Mater., 2005, 17, 2181-2185. (b) A. Saxena, R. Rai, S.-Y. Kim, M. Fujiki, M. Naito, K. Okoshi, G. Kwak, J. Polymer Sci.: Part A: Polymer Chem., 2006, 5060-5075. 
51 S. J. Toal, J. C. Sanchez, R. E. Dugan, W. C. Trogler, J. Forensic Sci., 2007, 52, 7983.

${ }^{52}$ R. C. Stringer, S. Gangopadhyay, S. A. Grant, Anal Chem, 2010, 82, 4015-4019.

${ }^{53}$ J. Lin, C. E. Kending, E. E. Nesterov, J. Am. Chem Soc. 2007, 129, 15911-15918.

${ }^{54}$ G. Bunte, J. Hürttlen, H. Pontius, K. Hartlieb, H. Krause, Anal. Chim. Acta, 2007, 591, 49-56.

55 a) X. Zhang, S. A. Jenekhe, Macromolecules, 2000, 33, 2069-2082. b) S. Hou, M. Ding, L. Gao, Macromolecules, 2003, 36, 3826-3832.

${ }^{56}$ T. H. Kin, H. J. Kim, C. G. Kwak, W. H. Park, T. S. Lee, J. Polym. Sci., Part A: Polym. Chem. 2006, 2059-2068.

${ }^{57}$ H. Nie, Y. Zhao, M. Zhang, Y. Ma, M. Baumgarten, K. Müllen, Chem. Commun, 2011, 47, 1234-1236.

58 A. Qin, J. W. Y. Lam, L. Tang, C. K. W. Jim, H. Zhao, J. Sun, B. Z. Tang, Macromolecules, 2009, 42, 1421-1424.

59 A. Kumar, M. K. Pandey, R. Anandakathir, R. Mosurkal, V. S. Parmar, A. C. Watterson, J. Kumar, Sensors and Actuators B, 2010, 147, 105-110.

60 H.H. Nguyen, X. Li, N. Wang, Z.Y. Wang, J. Ma, W.J. Bock, D. Ma, Macromolecules, 2009, 42, 921-926.

${ }^{61}$ K. J. Albert, D. R. Walt, Anal.Chem. 2000, 72, 1947-1955.

${ }^{62}$ D, Gao, Z. Wang, B. Liu, L. Ni, M. Wu, Z. Zhang, Anal. Chem. 2008, 80, 8545-8553.

${ }^{63}$ Q. Fang, J. Geng, B. Liu, D. Gao, F. Li, Z. Wang, G. Guan, Z. Zhang, Chem. Eur. J., 2009, 15, 11507-11514.

${ }^{64}$ J. Geng, P. Liu, B. Liu, G. Guan, Z. Zhang, M.-Y. Han, Chem. Eur. J., 2010, 16, $3720-3727$.

${ }^{65}$ J. Yang, S. Aschemeyer, H. P. Martínez, W. C. Trogler, Chem. Commun., 2010, 46, 6804-6806.

${ }^{66}$ J. Feng, Y. Li, M. Yang, Sensors \& Actuators, B, 2010, 145, 438-443.

${ }^{67}$ S. Tao, Z. Shi, G. Li, P. Li, ChemPhysChem., 2006, 7, 1902-1905.

${ }^{68}$ S. Tao, J. Yin, G. Li, J. Mat.Chem., 2008, 18, 4872-4878.

${ }^{69}$ S. Tao, G. Li, H. Zhu, J. Mat. Chem., 2006, 16, 4521-4528.

${ }^{70}$ S. Tao, G. Li, Colloid Polym. Sci. 2007, 285, 721-728.

${ }^{71}$ A. Yildirim, H. Budunoglu, H. Deniz, M. O. Guler, M. Bayindir, Appl. Mater. Interfaces, 2010, 2, 2892-2897. 
${ }^{72}$ H. Li, J. Wang, Z. Pan, L. Cui, L. Xu, R. Wang, Y. Song, L. Jiang, J. Mater. Chem., 2011, 21, 1730-1735.

${ }^{73}$ Z. Tao, G. Li, J. Yin, J. Mater.Chem., 2007, 17, 2730-2736.

${ }^{74}$ T. Naddo, Y. Che, W. Zhang, K. Balakrishnan, X. Yang, M. Yen, J. Zhao, J. S. Moore, L. Zang, J. Am. Chem. Soc., 2007, 129, 6978-6979.

${ }^{75}$ S. Content, W. C. Trogler, M. J. Sailor, Chem. Eur. J., 2000, 6, 2205-2213.

${ }^{76}$ J. Kang, L. Ding, F. Lü, S. Zhang, Y. Fang, J.Physics D: Appl. Phys., 2006, 39, 50975102.

${ }^{77}$ S. Zhang, F. Lü, L. Gao, L. Ding, Y. Fang, Langmuir, 2007, 23, 1584-1590.

${ }^{78}$ G. He, G. Zhang, F. Lü, Y. Fang, Chem.Mater., 2009, 21, 1494-1499.

${ }^{79}$ J. V. Goodpaster, V. L. McGuffin, Anal. Chem. 2001, 73, 2004-2011.

${ }^{80}$ A. D. Hughes, I. C. Glenn, A. D. Patrick, A. Ellington, E. V. Anslyn, Chem. Eur. J. 2008, 14, 1822-1827.

${ }^{81}$ S. Malashikhin, N. S. Finney, J. Am. Chem. Soc., 2008, 130, 12846-12847.

${ }^{82}$ K.-S. Focsaneanu, J. C. Scaiano, Photochem. Photobiol. Sci., 2005, 4, 817-821.

${ }^{83}$ Y. H. Lee, H. Liu, J. Y. Lee, S. H. Kim, S. K. Kim, J. L. Sessler, Y. Kim, J. S. Kim, Chem. Eur. J., 2010, 16, 5895-5901.

84 C. Jian, W. R. Seitz, Analytica Chimica Acta, 1990, 237, 265-271.

${ }^{85}$ C. Vijayakumar, G. Tobin, W. Schmitt, M. Kima, M. Takeuchi, Chem. Commun., 2010, 46, 874-876.

${ }^{86}$ G. V. Zyryanov, M. A. Palacios, P. Anzenbacher Jr, Org. Lett, 2008, 10, 3681-3684.

${ }^{87}$ H.Cavaye, P.E. Shaw, X. Wand, P.L. Burn, S-C Lo, P. Meredith, Macromolecules, 2010, 43, 10253-10261.

${ }^{88}$ A. Ponnu, E. V. Anslyn, Supramol. Chem., 2010, 22, 65-71.

${ }^{89}$ C. Zhang, Y. Che, X. Yang, B. R. Bunes, L. Zang, Chem. Commun., 2010, 46, 55605562 .

${ }^{90}$ Z. Li, Y. Q. Dong, J. W. Y. Lam, J. Sun, A. Qin, M. Haüßler, Y. P. Dong, H. H. Y. Sung, I. D. Williams, H. Sing Kwok, B. Z. Tang, Adv. Funct. Mater. 2009, 19, 905-917. ${ }^{91}$ Y. Dong, J.W.Y. Lam, A. Qin, Z. Li, J. Liu, J. Sun, Y. Dong, B. Z. Tang, Chem. Phys. Lett., 2007, 446, 124-127.

${ }^{92}$ L. Wang, Y. Zhou, J. Yan, J. Wang, J. Pei, Y. Cao., Langmuir, 2009, 25, 1306-1310.

${ }^{93}$ M. Rahman, H. J. Harmon, Spectrochim. Acta Part A, 2006, 65, 901-906.

94 D. A. Olley, E. J. Wren, G. Vamvounis, M. J. Fernee, X. Wang, P. L. Burn, P. Meredith, P. E. Shaw, Chem. Mater. 2011, 23, 789-794. 
95 S. Glazier, J.A. Barron, N. Morales, A. M. Ruschak, P. L. Houston, H. D. Abruña. Macromolecules, 2003, 36, 1272-1278.

96 S. Ghosh, P. S. Mukherjee, Organometallics, 2008, 27, 316-319.

97 S. Ghosh, B. Gole, A. K. Bar, P. S. Mukherjee, Organometallics, 2009, 28, 42884296.

${ }^{98}$ A. Kumar Bar, B. Gole, S. Ghosh, P. Sarathi Mukherjee, Dalton Trans., 2009, 67016704.

${ }^{99}$ M. E. Germain, T. R. Vargo, P. G. Khalifah, M. J. Knapp, Inorg. Chem. 2007, 46, 4422-4429.

${ }^{100}$ M. E. Germain, M. J. Knapp, J. Am. Chem. Soc., 2008, 130, 5422-5423.

${ }^{101}$ M. E. Germain, T. R. Vargo, B. A. McClure, J. J. Rack, P. G. Van Patten, M. Odoi, M. J. Knapp, Inorg. Chem. 2008, 47, 6203-6211.

${ }^{102}$ M. E. Germain, M. J. Knapp, Inorg. Chem., 2008, 47, 9748-9750.

${ }^{103}$ T. L. Andrew, T. M. Swager, J. Am. Chem. Soc., 2007, 129, 7254-7255.

${ }^{104}$ R. Schulte-Ladbeck, P. Kolla, U. Karst, Anal. Chem., 2003, 75, 731-735.

${ }^{105}$ E. Sella, D. Shabat, Chem. Commun., 2008, 5701-570.

106 J. C. Sanchez, S. J. Toal, Z. Wang, R. E. Dugan, W. C. Trogler, J. Forensic Sci., 2007, 52, 1308-1312.

${ }^{107}$ M. S. Meaney, V. L. McGuffin, Anal. Chim. Acta, 2008, 610, 57-67.

108 T. Caron, M. Guillemot, P. Montméat, F. Veignal, F. Perraut, P. Prené, F. SereinSpirau, Talanta, 2010, 81, 543-548.

${ }^{109}$ L. Cartmill, V. B. Rajpara, G. A. Sereda, J. Undergrad. Chem. Res., 2007, 6, 95-97.

${ }^{110}$ Y. Jiang, H. Zhao, N. Zhu, Y. Lin, P. Yu and L. Mao, Angew. Chem. Int. Ed., 2008, 47, 8601-8604.

111 S. S. R. Dasary, A. Kumar Singh, D. Senapati, H. Yu, P. C. Ray , J. Am. Chem. Soc., 2009, 131, 13806-13812.

112 D. S. Kim, V. M. Lynch, K. A. Nielsen, C. Johnsen, J. O. Jeppesen, J. L. Sessler, Anal. Bioanal. Chem., 2009, 395-400.

${ }^{113}$ K. A. Nielsen,W-S. Cho, J. O. Jeppesen, V. M. Lynch, J. Becher, J. L. Sessler, J. Am. Chem. Soc., 2004, 126, 16296-16297.

${ }^{114}$ J. Su Park, F. Le Derf, C. M. Bejger, V. M. Lynch, J. L. Sessler, K. A. Nielsen, C. Johnsen, J. O. Jeppesen, Chem. Eur. J., 2010, 16, 848-854.

${ }^{115}$ L. M. Dorozhkin, V. A. Nefedov, A. G. Sabelnikov, V. G. Sevastjanov, Sensors Act. $B, 2004,99,568-570$. 
116 A. Chen, H. Sun, A. Pyayt, X. Zhang, J. Luo, A. Jen, P. A. Sullivan, S. Elangovan, L. R. Dalton, R. Dinu, D. Jin, D. Huang, J. Phys. Chem. C, 2008, 112, 8072-8078.

${ }^{117}$ A. Ponnu, N. Y. Edwards, E. V. Anslyn, New J. Chem., 2008, 32, 848-855.

118 J. V. Janowski, Ber., 1886, 19, 2155.

${ }^{119}$ F. L. English, Anal. Chem., 1948, 20, 745-746.

120 S. A. H. Amas, H. J. Yallop, Analyst, 1966, 91, 336-337.

121 S. A. H. Amas, H. J. Yallop, J. Forens. Sci. Soc., 1966, 6, 185-188.

122 S. A. H. Amas, H. J. Yallop, Analyst, 1969, 94, 828.

${ }^{123}$ R. Schulte-Ladbeck, P. Kolla, U. Karst, Analyst, 2002, 127, 1152-1154.

124 Ş. Eren, A. Üzer, Z. Can, T. Kapudan, E. Erçağ, R. Apak. Analyst, 2010, 135, $2085-$ 2091.

${ }^{125}$ H. Lin, K. S. Suslick, J. Am. Chem. Soc, 2010, 132, 15519-15521.

126 E. S. Forzani, D. Lu, M. J. Leright, A. Diaz Aguilar, F. Tsow, R. A. Iglesias, Q. Zhang, J. Lu, J. Li, N. Tao, J. Am. Chem. Soc., 2009, 131, 1390-1391.

${ }^{127}$ C. J. McHugh, R. Keir, D. Graham, W. E. Smith, Chem. Commun., 2002, 580-581.

${ }^{128}$ Q. Lua, G. E. Collins, M. Smith, J. Wang, Anal. Chim. Acta, 2002, 469, 253-260.

129 J. Almog, A. Klein, T. Tamiri, Y. Shloosh, S. Abramovich-Bar, J. Forensic Sci., 2005, 50, 582-586. 